\title{
COLOR-MAGNITUDE DIAGRAMS FOR THREE DISTANT GLOBULAR CLUSTERS
}

\author{
J. G. COHEN \\ Palomar Observatory, California Institute of Technology, Pasadena, California 91125 \\ Received 17 January 1985; revised 5 August 1985
}

\begin{abstract}
Color-magnitude diagrams are presented for three distant galactic globular clusters (NGC 5466, NGC 6229, and NGC 7006). In each case the photometry reaches below the main-sequence turnoff; the ages deduced by comparison with stellar evolutionary isochrones are indistinguishable from those of the well-studied nearby clusters. The luminosity functions derived from computer-generated star counts complete to a well-defined limiting magnitude in selected fields support identical ages for the three clusters with maximum differences between NGC 5466 and NGC 6229 of $10 \%$ of the age, and between NGC 5466 and NGC 7006 of $25 \%$. The previously published claims of an exceptionally bright turnoff for NGC 7006 are shown to be spurious. The horizontal branch of NGC 6629 is unusually red for its metallicity. Star counts and tidal radii are discussed for NGC 6229 and NGC 7006.
\end{abstract}

\section{INTRODUCTION}

The halo globular clusters are of interest in problems of the formation and chemical evolution of the Galaxy. While the abundances of the halo clusters can be determined by analyzing their integrated light (see, for example, Zinn 1980; or Frogel, Persson, and Cohen 1983) or the spectra of their brighter giants, it is much more difficult to obtain information about the age of these clusters. This requires photometry reaching the main sequence, which for the more distant clusters in the outer halo of the Galaxy has until recently been impossible. Furthermore, the outer halo clusters are the most compressed in linear scale, making crowding problems more severe. We present here color-magnitude diagrams for three of the more distant galactic globular clusters (NGC 5466, NGC 6229, and NGC 7006). In each case we have succeeded in reaching the turnoff. We demonstrate that an earlier claim of a very young age for NGC 7006 is spurious. The luminosity functions for the three clusters are discussed in Sec. III $b$, while the horizontal-branch morphology of these clusters in the outer galactic halo is discussed in Sec. III $c$. Star counts and tidal radii for two of the clusters are discussed in Sec. IV. A summary of our conclusions is given in Sec. $\mathrm{V}$.

\section{OBSERVATIONAL MATERIAL}

\section{a) Instrumental Magnitudes}

Frames of three distant globulars (NGC 5466, 6229, and 7006) were obtained in August 1984, March 1985, and April 1985 on the $5 \mathrm{~m}$ Hale telescope at Palomar Observatory using the 4-Shooter (see Gunn et al. 1984). The scale of the 4Shooter is $0.33 \mathrm{arcsec} / \mathrm{pixel}$. The seeing at the zenith was slightly better than 1.0 arcsec and the sky was photometric in the first two observing runs. Several frames were taken of each cluster as listed in Table I through the $g$ and $r$ filters of the Thuan and Gunn (1976) system. The $g$ and $r$ magnitudes were calibrated using out-of-focus exposures of the bright Thuan-Gunn standards. Standard extinction coefficients for Palomar were used. Short exposurés were taken to find guide stars, and all the longer exposures were guided. The central core of each cluster is hopelessly confused (except for NGC 5466), but in the outer parts there is little or no overlap of the stellar images. However, even the outer parts are too crowded for standard aperture photometry. The procedure adopt- ed was to isolate several small (less than 2 arcmin on a side) regions free of cosmetic defects in the CCDs on the periphery of each cluster, where the crowding was tolerable. The locations of the fields with respect to the cluster centers are given in Table II, and the fields are shown in Figs. 1-3 [Plates 244 246] (made from the $600 \mathrm{~s} g$ frames). In each field, a star is overlaid with a cursor (a cross) whose location and magnitude is also listed in Table II.

A list of the point sources whose central pixel was brighter than a predetermined threshold level was compiled for each exposure of each of the small fields. Sources with any saturated pixels were eliminated. The sky level was chosen by sampling regions free of stars within each of the fields and was taken as constant within each region. The sum of the detected CCD counts in a $5 \times 5$ pixel box centered on the point-source maximum (minus 25 times the mean sky for the region) was used to calculate the "box magnitudes" for each object. A selection of ten well-isolated stars in each field was used to determine the magnitude difference between largeaperture photometry and the box magnitude defined above. In the most crowded regions, only seven well-isolated objects could be found. Because the seeing was good, this offset was in the best cases only $0.12 \mathrm{mag}$ and in the worst case (NGC 5466, observed at a large airmass in August 1984) was 0.4 mag. The dispersion in this offset within a given field as

TABLE I. Frames of NGC 5466, NGC 6229, and NGC 7006

\begin{tabular}{lcc}
\hline \hline Object & $\begin{array}{c}g \\
(\mathbf{s})\end{array}$ & $\begin{array}{c}r \\
(\mathbf{s})\end{array}$ \\
\hline NGC 5466 & 40 & 200 \\
& 300 & 600 \\
& 300 & - \\
NGC 6229 & 600 & - \\
& 136 & 200 \\
& 200 & 600 \\
NGC 7006 & 600 & 800 \\
& 800 & - \\
& 800 & 100 \\
& 600 & 600 \\
& $800^{\mathrm{a}}$ & - \\
\hline \hline
\end{tabular}

a Nonphotometric night. 
TABLE II. Field centers.

\begin{tabular}{|c|c|c|c|}
\hline Name & $\begin{array}{l}(1,1) \text { Relative to } \\
\text { cluster center } \\
\text { (pixels) }\end{array}$ & $\begin{array}{l}\text { Cursor } \\
\text { (pixels) }\end{array}$ & $\underset{(\mathrm{mag})}{g}$ \\
\hline \multicolumn{4}{|c|}{ NGC 5466} \\
\hline $\begin{array}{l}\text { Field } 1 \\
\text { Field } 2 \\
\text { Field } 3 \\
\text { Field } 4 \\
\text { Field } 5 \\
\text { Field } 6\end{array}$ & $\begin{array}{r}-180,-600 \\
-180,-250 \\
300,-450 \\
300, \quad 250 \\
100, \quad 400 \\
-100, \quad 500\end{array}$ & $\begin{array}{r}208,46 \\
- \\
92,68 \\
109,47 \\
88,70 \\
115,73\end{array}$ & $\begin{array}{l}20.60 \\
20.27 \\
20.16 \\
20.17 \\
19.32\end{array}$ \\
\hline \multicolumn{4}{|c|}{ NGC 6229} \\
\hline $\begin{array}{l}\text { Field } 1 \\
\text { Field } 3 \\
\text { Field } 4 \\
\text { Field } 5 \\
\text { Field } 6\end{array}$ & $\begin{array}{r}-120,-500 \\
-320,-500 \\
-130, \quad 580 \\
100, \quad 580 \\
150,-500\end{array}$ & $\begin{array}{r}150,191 \\
54,115 \\
83,163 \\
30,152 \\
22,158\end{array}$ & $\begin{array}{l}20.82 \\
21.73 \\
21.56 \\
20.89 \\
21.50\end{array}$ \\
\hline \multicolumn{4}{|c|}{ NGC 7006} \\
\hline $\begin{array}{l}\text { Field } 1 \\
\text { Field } 2 \\
\text { Field } 3\end{array}$ & $\begin{array}{rr}110, & 260 \\
120,-570 \\
-470, & 260\end{array}$ & $\begin{array}{c}130,50 \\
77,8 \\
89,28\end{array}$ & $\begin{array}{l}20.12 \\
21.77 \\
21.82\end{array}$ \\
\hline
\end{tabular}

determined from well-isolated stars was $0.08 \mathrm{mag}$, which presumably arises from the statistics of the placement of the center of the image with respect to the center of a pixel.

The point-source detections in each of the frames of a given field were merged by a computer program, which also took into account the small shifts between frames. When there was more than one frame of a field in any given color, the magnitudes were averaged and the dispersions about the means calculated. The only manual intervention in the whole procedure was the elimination of small resolved galaxies (a maximum of three per region), and the selection of well-isolated stars and subsequent calculation of the offsets between the box magnitudes and true large-aperture magnitudes.

A discussion of the difference between the CCD/PFUEI instrumental system and the photoelectric photometry that defines the magnitudes and colors of the Thuan-Gunn system is given in Schneider, Gunn, and Hoessel (1983). However, the 4-shooter has significantly better blue response than does the PFUEI (although they both use the same $800 \times 800$ Texas Instruments CCD chips as detectors). Thus the transformation between our instrumental and the photoelectric $g-r$ colors has a slope significantly closer to 1.0 than was found by Schneider et al. This transformation was calculated from two photometric nights during each of which six bright Thuan-Gunn photometric standards were observed. The standards span the range of $g-r$ color from -0.79 to $0.57 \mathrm{mag}$. The final averaged $g$ and $r$ magnitudes are listed in Tables III-V. ${ }^{* \dagger}$ As one can see from looking at the magnitude dispersions, which are listed in the tables in units of $0.01 \mathrm{mag}$, except in those cases where the matchup

\footnotetext{
The five fields of NGC 6229 gave color-magnitude diagrams slightly offset from each other. These fields occurred on different chips of the 4-Shooter (as was the case for all three clusters) and each field was not always on the same chip in all the frames. The origin of this problem is unknown, but to eliminate it small offsets were made in all the $g$ magnitudes for a given field in NGC 6229 only. The offsets did not exceed $0.05 \mathrm{mag}$, and the average of the offsets for the five fields was set equal to zero.

${ }^{\dagger}$ Seven stars were omitted from the end of Table III to avoid having an extra page in the table with only two lines of data.
}

between detected point sources in different frames failed (presumably due to crowding), the dispersions are quite reasonable. At these faint magnitudes, the crowding and the uncertainties in flattening of the sky dominate the errors.

\section{b) Transformation to $B, V$}

The transformation from $g, r$, to $B, V$ was made using the bright standards of the Thuan-Gunn system with $B, V$ magnitudes in Blanco et al. (1968). The standards were fit with the relationships

$$
\begin{aligned}
& V=g-0.371(g-r)-0.038 \\
& B-V=0.454+0.963(g-r) .
\end{aligned}
$$$$
\text { and }
$$

The dispersion of the difference between the predicted and published $V$ and $B-V$ magnitudes was $0.02 \mathrm{mag}$, with the largest absolute value of a difference being 0.04 mag. These transformations have been used throughout this paper.

As a check of these transformations, a field in the periphery of M3 with a faint sequence by Sandage (1970) was observed in March 1985. The $B, V$ magnitudes were calculated from the $g, r$ magnitudes and compared with those of Sandage. For 12 stars with $16 \leqslant V \leqslant 21 \mathrm{mag}$, the mean difference in $V$ was $0.01 \mathrm{mag}$ with a dispersion of $0.09 \mathrm{mag}$, while the mean difference in $B-V$ was 0.085 mag with a dispersion of 0.10 mag. The five photoelectric standards from Sandage and Wildey's (1967) study of NGC 7006 that were faint enough to be unsaturated in our shortest $g$ and $r$ frames were also used to check the transformation of photometric systems. A mean difference between $V$ (predicted) and $V$ (Sandage-Wildey) of $0.05 \mathrm{mag}$ with a dispersion of $0.10 \mathrm{mag}$ and a mean $B-V$ difference of $0.06 \mathrm{mag}$ with a dispersion of 0.04 mag was found.

We note that our $g$ magnitudes for the faintest of Baade's (1945) standards in NGC 6229 have a mean $\left(m_{p g}-g\right)$ of 0.01 mag with a dispersion about the mean of $0.24 \mathrm{mag}$. (Standard $r$ of Baade appears to be misidentified on his plate II and we ignore it.)

\section{CLUSTER AGES}

\section{a) Isochrone Fits}

Figures 4-8 show the $g, r$, color-magnitude diagrams for the three distant halo clusters. No effort has been made to remove the known RR Lyrae variables from any of the figures. All point sources measured on at least two of the $g$ frames and one of the $r$ frames of NGC 5466 in fields 1 to 6 (excluding field 2, which is too crowded for reliable photometry) that are brighter than $r=22.0$ are plotted in Fig. 4. For NGC 6229, all points in the five outlying fields studied with $r \leqslant 23.50$ mag that were measured on at least three of the $g$ frames and two of the $r$ frames are shown in Fig. 5. The solid line represents the mean locus of the brighter subgiants picked up in a search of an area on the frames closer to the center of NGC 6229, where crowding limits the photometry to the brightest stars only; the color-magnitude diagram for these stars is shown in Fig. 6. Figure 7 shows the stars detected on at least one of the $g$ and $r$ frames taken on photometric nights in fields close to the center of the cluster NGC 7006, where faint photometry is impossible because of crowding. Even at the magnitude levels shown, crowding has limited the accuracy of the magnitudes to $\pm 0.10 \mathrm{mag}$. Stars detected on at least two of the $g$ frames and one of the $r$ frames that are brighter than $r=23.50 \mathrm{mag}$ in three small fields on the 
TABLE III. Magnitudes of NGC 5466 stars.

\begin{tabular}{|c|c|c|c|c|c|c|c|c|c|c|c|c|c|c|c|c|c|c|c|c|c|c|c|}
\hline & ix) & $r$ & g-r & \# & $\begin{array}{c}\sigma(r) \\
(\mathrm{mag})\end{array}$ & $\#$ & $\begin{array}{c}\sigma(g) \\
(\mathrm{mag})\end{array}$ & & ix) & $\mathrm{r}$ & g-r & $\#$ & $\begin{array}{c}\sigma(r) \\
(\mathrm{mag})\end{array}$ & \# & $\begin{array}{c}\sigma(g) \\
(\mathrm{mag})\end{array}$ & & ix) & $\mathbf{r}$ & g-r & $\#$ & $\begin{array}{c}\sigma(r) \\
\text { (mag) }\end{array}$ & & $\begin{array}{c}\sigma(g) \\
(\mathrm{mag})\end{array}$ \\
\hline & Field & & & & & & & 153 & 1 & 21.93 & 0.65 & 1 & 0 & 2 & 1 & 232 & 1 & 19.73 & 0.04 & 1 & 0 & 3 & 1 \\
\hline 265 & 1 & 20.52 & 0.20 & 1 & 0 & 3 & 0 & 77 & 12 & 20.97 & 0.08 & 2 & 4 & 4 & 9 & 120 & 13 & 21.55 & 0.28 & 2 & 0 & 3 & 17 \\
\hline 239 & 16 & 21.04 & 1.14 & 2 & 0 & 3 & 9 & 140 & 26 & 21.47 & 0.04 & 2 & 3 & 3 & 11 & 226 & 29 & 20.86 & 0.05 & 2 & 0 & 4 & 2 \\
\hline 10 & 31 & 21.84 & -0.44 & 2 & 53 & 3 & 3 & 88 & 31 & 19.37 & 0.17 & 2 & 0 & 4 & 4 & 75 & 36 & 21.44 & 0.16 & 2 & 7 & 3 & 8 \\
\hline 198 & 37 & 21.21 & 1.10 & 2 & 2 & 3 & 21 & 220 & 41 & 19.88 & 0.07 & 2 & 2 & 4 & 4 & 111 & 46 & 21.76 & 0.35 & 2 & 1 & 3 & 10 \\
\hline 208 & 46 & 20.53 & 0.07 & 2 & 4 & 4 & 3 & 260 & 52 & 21.85 & 0.32 & 2 & 2 & 3 & 13 & 156 & 53 & 21.00 & 0.12 & 2 & 0 & 3 & 8 \\
\hline 9 & 64 & 19.65 & 0.05 & 2 & 1 & 4 & 2 & 252 & 64 & 21.73 & 0.28 & 2 & 10 & 3 & 18 & 168 & 66 & 20.50 & 0.03 & 2 & 2 & 4 & 3 \\
\hline 241 & 73 & 21.64 & 0.18 & 2 & 12 & 3 & 9 & 131 & 77 & 19.35 & 0.15 & 2 & 1 & 4 & 2 & 67 & 78 & 21.27 & 0.06 & 2 & 0 & 3 & 6 \\
\hline 289 & 78 & 21.43 & 0.73 & 2 & 8 & 3 & 9 & 153 & 81 & 20.59 & 0.01 & 2 & 1 & 4 & 2 & 77 & $84^{\circ}$ & 21.68 & 0.14 & 2 & 2 & 3 & 5 \\
\hline 8 & 89 & 20.49 & 0.00 & 2 & 1 & 4 & 2 & 171 & 90 & 19.76 & 0.48 & 2 & 2 & 4 & 1 & 247 & 92 & 21.25 & 0.09 & 2 & 9 & 3 & 6 \\
\hline 189 & 93 & 21.35 & 0.13 & 2 & 4 & 3 & 5 & 111 & 96 & 21.13 & 0.05 & 2 & 1 & 3 & 3 & 151 & 102 & 17.64 & 0.23 & 1 & 0 & 3 & 0 \\
\hline 285 & 102 & 21.76 & 0.19 & 2 & 8 & 3 & 8 & 196 & 106 & 20.60 & -0.02 & 2 & 5 & 4 & 2 & 275 & 106 & 19.58 & 0.04 & 2 & 5 & 3 & 2 \\
\hline 8 & 108 & 20.57 & 0.00 & 2 & 3 & 3 & 3 & 108 & 111 & 21.92 & 0.15 & 2 & 3 & 2 & 2 & 140 & 113 & 21.31 & 0.00 & 2 & 6 & 2 & 0 \\
\hline 256 & 113 & 21.06 & 0.15 & 2 & 5 & 2 & 11 & 9 & 116 & 20.70 & -0.01 & 2 & 2 & 3 & 3 & 72 & 116 & 20.40 & 0.03 & 2 & 2 & 3 & 3 \\
\hline 87 & 116 & 19.77 & -0.21 & 2 & 1 & 3 & 3 & 240 & 117 & 21.71 & 0.26 & 2 & 2 & 2 & 14 & 115 & 120 & 21.39 & 0.06 & 2 & 0 & 3 & 8 \\
\hline 133 & 120 & 21.13 & 0.04 & 2 & 1 & 3 & 1 & 202 & 124 & 21.37 & 0.22 & 2 & 1 & 3 & 7 & 215 & 124 & 20.39 & 0.03 & 2 & 2 & 4 & 0 \\
\hline 235 & 124 & 21.44 & 0.18 & 2 & 2 & 3 & 4 & 12 & 125 & 21.22 & 0.08 & 2 & 4 & 3 & 5 & 147 & 125 & 21.22 & 0.14 & 2 & 3 & 3 & 1 \\
\hline 58 & 126 & 21.65 & 0.09 & 2 & 2 & 3 & 3 & 25 & 127 & 18.57 & 0.24 & 1 & 0 & 4 & 2 & 270 & 127 & 20.79 & 0.07 & 2 & 3 & 4 & 6 \\
\hline 218 & 133 & 21.29 & 0.16 & 2 & 1 & 3 & 2 & 26 & 137 & 21.78 & 0.17 & 1 & 0 & 2 & 3 & 192 & 140 & 20.82 & 0.03 & 2 & 2 & 4 & 5 \\
\hline 218 & 144 & 20.00 & -0.01 & 2 & 3 & 4 & 1 & 12 & 147 & 21.75 & 0.09 & 2 & 3 & 3 & 8 & 123 & 147 & 20.74 & 0.00 & 2 & 0 & 4 & 5 \\
\hline 30 & 148 & 21.93 & -0.05 & 2 & 14 & 2 & 18 & 55 & 148 & 21.03 & -0.09 & 2 & 5 & 3 & 5 & 256 & 148 & 20.39 & 0.05 & 2 & 3 & 4 & 4 \\
\hline 219 & 149 & 20.90 & 0.04 & 2 & 1 & 2 & 0 & 150 & 155 & 20.73 & 0.10 & 2 & 0 & 4 & 5 & 188 & 155 & 21.56 & 0.34 & 2 & 3 & 3 & 4 \\
\hline 209 & 155 & 20.98 & 0.08 & 2 & 0 & 3 & 3 & 273 & 157 & 19.53 & 0.05 & 2 & 6 & 4 & 2 & 284 & 157 & 21.18 & 0.19 & 2 & 4 & 3 & 4 \\
\hline 145 & 160 & 20.77 & 0.04 & 2 & 3 & 4 & 7 & 217 & 161 & 21.06 & 0.09 & 2 & 3 & 3 & 1 & 248 & 165 & 20.71 & 0.07 & 2 & 1 & 4 & 0 \\
\hline 224 & 168 & 20.95 & 0.02 & 2 & 2 & 3 & 1 & 58 & 171 & 21.73 & 0.08 & 2 & 1 & 3 & 6 & 157 & 172 & 21.79 & 0.03 & 2 & 0 & 3 & 10 \\
\hline 12 & 173 & 18.55 & 0.28 & 1 & 0 & 4 & 2 & 269 & 173 & 19.82 & 0.04 & 2 & 5 & 4 & 1 & 87 & 175 & 20.82 & 0.01 & 2 & 1 & 3 & 2 \\
\hline 41 & 179 & 20.88 & 0.01 & 2 & 2 & 3 & 2 & 158 & 179 & 20.78 & -0.02 & 2 & 1 & 3 & 5 & 261 & 180 & 21.77 & 0.16 & 2 & 1 & 3 & 1 \\
\hline 105 & 181 & 20.03 & 0.03 & 2 & 1 & 4 & 6 & 222 & 183 & 21.76 & 0.16 & 2 & 0 & 3 & 7 & 293 & 183 & 20.49 & 0.08 & 1 & 0 & 4 & 2 \\
\hline 30 & 185 & 21.59 & 0.10 & 2 & 3 & 3 & 8 & 67 & 186 & 21.71 & 0.14 & 2 & 8 & 3 & 5 & 120 & 186 & 20.92 & -0.12 & 2 & 12 & 3 & 14 \\
\hline 96 & 187 & 21.68 & 0.02 & 2 & 0 & 3 & 6 & 254 & 189 & 21.75 & 0.32 & 2 & 2 & 3 & 5 & 56 & 190 & 21.19 & 0.00 & 2 & 3 & 3 & 5 \\
\hline 229 & 191 & 21.63 & 0.10 & 2 & 4 & 3 & 0 & 40 & 192 & 20.71 & -0.04 & 2 & 4 & 4 & 4 & 183 & 192 & 19.89 & 0.00 & 2 & 0 & 4 & 3 \\
\hline 35 & 197 & 19.96 & 0.00 & 2 & 3 & 4 & 4 & 257 & 198 & 21.44 & 0.21 & 2 & 0 & 3 & 9 & 7 & 199 & 20.60 & -0.01 & 2 & 2 & 4 & 5 \\
\hline 183 & 202 & 21.69 & 0.13 & 2 & 2 & 3 & 4 & 18 & 203 & 20.93 & 0.01 & 2 & 4 & 3 & 2 & 287 & 204 & 21.26 & 0.14 & 2 & 2 & 3 & 2 \\
\hline 294 & 204 & 21.96 & 0.22 & 1 & 0 & 3 & 1 & 6 & 206 & 20.15 & 0.01 & 2 & 2 & 4 & 4 & 140 & 206 & 21.87 & 0.22 & 2 & 8 & 3 & 18 \\
\hline 195 & 207 & 21.36 & 0.09 & 2 & 1 & 2 & 13 & 181 & 208 & 20.33 & 0.03 & 2 & 2 & 4 & 7 & 52 & 213 & 21.85 & 0.06 & 2 & 3 & 3 & 9 \\
\hline 43 & 214 & 19.35 & 0.03 & 2 & 2 & 4 & 3 & 74 & 216 & 21.85 & 0.20 & 2 & 3 & 3 & 4 & 169 & 218 & 21.80 & 0.03 & 2 & 0 & 3 & 11 \\
\hline 277 & 221 & 21.52 & 0.64 & 2 & 39 & 2 & 24 & 257 & 222 & 19.75 & 0.05 & 2 & 0 & 4 & 1 & 32 & 224 & 21.94 & 0.08 & 2 & 2 & 3 & 9 \\
\hline 122 & 225 & 20.95 & -0.01 & 2 & 14 & 3 & 5 & 292 & 225 & 21.82 & 0.43 & 1 & 0 & 3 & 2 & 223 & 227 & 20.18 & 0.04 & 2 & 5 & 4 & 7 \\
\hline 244 & 227 & 21.81 & 0.25 & 2 & 0 & 3 & 6 & 280 & 230 & 17.36 & 1.29 & 1 & 0 & 4 & 2 & 168 & 231 & 21.86 & 0.06 & 2 & 15 & 3 & 19 \\
\hline 51 & 232 & 20.63 & 0.07 & 2 & 2 & 4 & 5 & 16 & 234 & 20.92 & 0.04 & 2 & 0 & 3 & 3 & 156 & 234 & 20.44 & 0.04 & 2 & 5 & 4 & 9 \\
\hline 92 & 235 & 21.64 & 0.10 & 2 & 0 & 3 & 8 & 230 & 235 & 19.40 & 0.13 & 2 & 1 & 4 & 5 & 58 & 236 & 21.23 & 0.01 & 2 & 2 & 3 & 4 \\
\hline 187 & 236 & 20.32 & 0.05 & 2 & 0 & 4 & 6 & 74 & 237 & 20.77 & 0.09 & 2 & 0 & 4 & 6 & 86 & 237 & 21.71 & 0.10 & 2 & 1 & 3 & 9 \\
\hline 150 & 237 & 21.41 & -0.01 & 2 & 22 & 3 & 19 & 174 & 237 & 19.93 & 0.02 & 2 & 0 & 4 & 5 & 203 & 237 & 21.27 & 0.13 & 2 & 2 & 3 & 5 \\
\hline 292 & 239 & 20.85 & 0.12 & 1 & 0 & 3 & 1 & 234 & 240 & 20.12 & -0.04 & 2 & 0 & 4 & 6 & 96 & 242 & 21.08 & -0.30 & 2 & 36 & 3 & 2 \\
\hline 135 & 242 & 20.81 & 0.12 & 1 & 0 & 3 & 18 & 223 & 242 & 21.87 & 0.08 & 2 & 10 & 3 & 13 & 199 & 244 & 21.33 & 0.06 & 2 & 1 & 3 & 9 \\
\hline 232 & 244 & 19.86 & 0.05 & 2 & 0 & 4 & 8 & 59 & 246 & 21.50 & 0.06 & 2 & 2 & 3 & 8 & 93 & 246 & 21.33 & 0.12 & 2 & 3 & 3 & 5 \\
\hline 101 & 246 & 21.72 & -0.06 & 1 & 0 & 3 & 9 & 23 & 248 & 17.39 & 0.30 & 1 & 0 & 3 & 2 & 141 & 250 & 21.51 & 0.04 & 2 & 2 & 3 & 15 \\
\hline 110 & 251 & 21.19 & 0.09 & 2 & 2 & 3 & 8 & 180 & 252 & 19.90 & 0.03 & 2 & 1 & 4 & 5 & 131 & 253 & 21.52 & 0.07 & 2 & 5 & 3 & 11 \\
\hline 121 & 255 & 21.59 & 0.20 & 2 & 3 & 3 & 7 & 189 & 257 & 21.35 & 0.05 & 2 & 1 & 3 & 5 & 66 & 258 & 19.11 & 0.19 & 2 & 2 & 4 & 4 \\
\hline
\end{tabular}


TABLE III. (continued)

\begin{tabular}{|c|c|c|c|c|c|c|c|c|c|c|c|c|c|c|c|c|c|c|c|c|c|c|c|}
\hline & ix) & $\mathrm{r}$ & $-\mathbf{r}$ & \# & $\begin{array}{c}\sigma(r) \\
(\mathrm{mag})\end{array}$ & $\neq$ & $\begin{array}{c}\sigma(g) \\
(\mathrm{mag})\end{array}$ & & $\mathrm{y}$ & $r$ & g-r & & $\begin{array}{c}\sigma(r) \\
(\mathrm{mag})\end{array}$ & & $\begin{array}{c}\sigma(g) \\
(\mathrm{mag})\end{array}$ & & ix) & $\mathbf{r}$ & g-r & & $\begin{array}{c}\sigma(r) \\
(\mathrm{mag})\end{array}$ & & $\begin{array}{c}\sigma(g) \\
(\mathrm{mag})\end{array}$ \\
\hline 55 & 258 & 20.80 & 0.08 & 2 & 1 & 4 & 7 & 223 & 259 & 19.94 & 0.02 & 2 & 1 & 4 & 4 & 202 & 260 & 21.84 & 0.16 & 2 & 0 & & 6 \\
\hline 75 & 260 & 21.29 & 0.14 & 2 & 4 & 3 & 4 & 154 & 261 & 21.56 & 0.19 & & 1 & & 11 & 62 & 261 & 21.40 & 0.06 & & 2 & & 9 \\
\hline 15 & 262 & 20.88 & 0.01 & 2 & 1 & 3 & 4 & 295 & 263 & 19.50 & 0.26 & & 0 & & 4 & 130 & 264 & 20.84 & 0.02 & & 2 & & 6 \\
\hline 90 & 265 & 21.06 & 0.06 & 2 & 2 & 2 & 3 & 12 & 272 & 21.17 & 0.12 & & 0 & & 1 & 273 & 272 & 21.20 & 0.10 & & 2 & & 3 \\
\hline 213 & 273 & 20.47 & -0.01 & 2 & 1 & 4 & 6 & 102 & 276 & 19.48 & 0.14 & 2 & 1 & & 5 & 50 & 279 & 20.22 & 0.03 & 2 & 1 & & 1 \\
\hline 30 & 279 & 20.97 & 0.02 & 2 & 1 & 3 & 6 & 214 & 281 & 20.65 & 0.15 & & 2 & & 14 & 255 & 282 & 19.52 & 0.07 & & 2 & & 7 \\
\hline 287 & 282 & 20.04 & 0.08 & 2 & 2 & 4 & 8 & 193 & 284 & 21.07 & 0.06 & 2 & 5 & & 10 & 76 & 286 & 20.80 & 0.08 & 2 & 3 & & 2 \\
\hline 292 & 286 & 20.96 & 0.12 & 1 & 0 & 3 & 2 & 141 & 287 & 21.41 & 0.08 & & 0 & & 7 & 24 & 288 & 21.51 & 0.19 & 2 & & & 5 \\
\hline 50 & 289 & 21.56 & 0.14 & 2 & 5 & 3 & 13 & 38 & 292 & 21.59 & 0.08 & & 7 & & 10 & 7 & 294 & 20.18 & 0.04 & & 2 & & 3 \\
\hline 56 & 295 & 19.32 & 0.12 & 2 & 2 & 3 & 4 & 236 & 296 & 21.77 & 0.21 & & 0 & & 5 & 20 & 297 & 20.94 & -0.05 & & 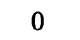 & & 1 \\
\hline 190 & 297 & 20.59 & -0.09 & 2 & 4 & 2 & 0 & & & & & & & & & & & & & & & & \\
\hline & Field & & & & & & & 54 & 11 & 20.78 & 0.04 & 2 & 0 & - & 9 & 100 & 20 & 21.86 & 0.40 & 2 & 3 & & 22 \\
\hline 93 & 21 & 19.99 & 0.00 & 2 & 2 & 4 & 3 & 58 & 26 & 19.90 & 0.01 & 2 & 2 & 4 & 2 & 121 & 26 & 20.61 & 0.07 & 2 & 1 & & 3 \\
\hline 23 & 27 & 21.36 & .09 & 2 & 1 & 3 & 6 & 12 & 31 & 21.38 & 0.19 & & 2 & & 9 & 53 & 31 & 45 & -0.01 & 2 & 1 & & 6 \\
\hline 71 & 31 & 20.83 & 0.09 & 2 & 5 & 4 & 8 & 125 & 33 & 20.49 & 0.07 & 2 & 2 & 4 & 4 & 110 & 40 & 21.31 & 0.22 & 2 & 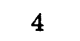 & & 12 \\
\hline 20 & 40 & 21.21 & 0.15 & 2 & 1 & 3 & 10 & 72 & 55 & 20.31 & 0.02 & 2 & 4 & & 6 & 39 & 58 & 21.93 & 0.35 & & 3 & & 18 \\
\hline 11 & 59 & 20.98 & 14 & 1 & 0 & 4 & 10 & 16 & 60 & 21.09 & 0.14 & 2 & 7 & 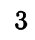 & 19 & 111 & 61 & 21.66 & 0.73 & 1 & 0 & & 1 \\
\hline 45 & 64 & 20.56 & 0.65 & 2 & 2 & 3 & 8 & 104 & 65 & 20.63 & 0.07 & 2 & 3 & 4 & 0 & 92 & 68 & 20.21 & 0.06 & 2 & 3 & & 6 \\
\hline 9 & 77 & 20.31 & -0.02 & 2 & 5 & 4 & 3 & 47 & 77 & 20.38 & 1.07 & & 3 & & 13 & 58 & 82 & 1 & 0.08 & 2 & 4 & & 5 \\
\hline 128 & 87 & 20.80 & 0.07 & 2 & 4 & 4 & 5 & 107 & 90 & 21.11 & 0.15 & 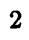 & 5 & 3 & 7 & 88 & 91 & 21.82 & 0.52 & 2 & 8 & & 18 \\
\hline 128 & 95 & 21.80 & 0.29 & 2 & 7 & 3 & 20 & 133 & 97 & 21.33 & 0.13 & & 0 & & 10 & 18 & 98 & 19.92 & 0.04 & & 4 & & 7 \\
\hline 81 & 100 & 21.59 & 0.49 & 2 & 5 & 3 & 16 & 34 & 101 & 20.34 & 0.05 & 2 & 2 & 4 & 3 & 54 & 102 & 19.97 & -0.01 & 2 & 0 & & 3 \\
\hline 109 & 103 & 21.90 & 0.19 & 2 & 12 & 3 & 12 & 105 & 109 & 21.73 & 0.15 & 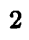 & 6 & & 6 & 34 & 122 & 18.60 & 0.18 & 1 & 0 & & 2 \\
\hline 72 & 122 & 19.77 & 0.04 & 2 & 0 & 4 & 2 & 106 & 125 & 21.48 & 0.23 & 2 & 8 & 3 & 3 & 5 & 133 & 20.31 & 0.12 & 1 & 0 & & 3 \\
\hline 99 & 134 & 21.29 & 0.15 & 2 & 0 & 3 & 4 & 26 & 137 & 21.57 & -0.05 & 2 & 25 & & 11 & 9 & 140 & 2 & 0.00 & 2 & 1 & & 2 \\
\hline 53 & 142 & 21.83 & 0.30 & 2 & 0 & 3 & 8 & 105 & 145 & 18.77 & 0.22 & & 0 & & 1 & 77 & 148 & 70 & 0.05 & 2 & c & & 1 \\
\hline 10 & 150 & 21.75 & 0.18 & 1 & 0 & 3 & 20 & 57 & 151 & 20.54 & 0.09 & 2 & 0 & & 9 & 75 & 156 & 20.69 & 0.04 & 2 & 1 & & 3 \\
\hline 67 & 158 & 21.10 & 0.07 & 2 & 1 & 3 & 2 & 137 & 163 & 21.80 & 0.20 & & 0 & & 0 & 92 & 175 & 20.99 & 0.01 & 2 & 2 & & 1 \\
\hline 49 & 176 & 19.52 & 0.06 & 2 & 1 & 3 & 4 & 113 & 179 & 21.45 & 0.06 & 2 & 2 & 3 & 0 & 120 & 185 & 18.22 & 0.26 & 1 & 0 & & 2 \\
\hline 56 & 191 & 20.34 & 0.04 & 2 & 0 & 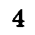 & 10 & 21 & 192 & 20.53 & 0.07 & & 2 & & 8 & 9 & 195 & 2 & 77 & 1 & 0 & & 9 \\
\hline 42 & 199 & 21.06 & -2.20 & 2 & 4 & 2 & 0 & 17 & 200 & 20.93 & 0.09 & 2 & 4 & 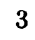 & 1 & 59 & 202 & 20.16 & -0.01 & 2 & 2 & & 4 \\
\hline 38 & 203 & 19.17 & 0.26 & 2 & 1 & 2 & 5 & 129 & 203 & 21.07 & -0.03 & 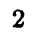 & 4 & & 8 & 05 & 207 & 21 & 0.13 & 2 & 1 & & 4 \\
\hline 129 & 212 & 20.47 & 0.02 & 2 & 2 & 4 & 9 & 23 & 215 & 20.59 & -0.01 & 2 & 3 & 3 & 5 & 124 & 218 & 21.08 & -0.03 & 2 & 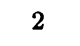 & & 7 \\
\hline 67 & 219 & 21.86 & -0.20 & 2 & 19 & 3 & 1 & 12 & 225 & 21.52 & 0.13 & 2 & 0 & & 3 & 42 & 227 & 21.16 & 0.26 & 2 & 10 & 3 & 4 \\
\hline 69 & 228 & 21.41 & 0.13 & 2 & 5 & 3 & 6 & 50 & 229 & 20.21 & 0.03 & 2 & 1 & & 9 & 112 & 231 & 21.67 & 0.19 & 2 & 6 & & 24 \\
\hline 133 & 233 & 20.04 & 0.09 & 1 & 0 & 4 & 3 & 53 & 235 & 21.35 & -0.01 & 2 & 3 & 3 & 7 & 94 & 242 & 19.83 & 0.02 & 2 & 2 & 4 & 3 \\
\hline 70 & 244 & 21.28 & ( & 2 & 2 & 3 & 9 & 106 & 248 & 21.51 & -0.03 & 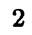 & 3 & & 15 & 37 & 250 & & 0.26 & 2 & 7 & & 8 \\
\hline 52 & 250 & 21.99 & 0.26 & 2 & 3 & 3 & 3 & 18 & 253 & 20.37 & 0.03 & 2 & 3 & 4 & 5 & 97 & 255 & 20.70 & 0.03 & 2 & 5 & 4 & 12 \\
\hline 41 & 256 & 21.55 & 0.28 & 2 & 6 & 3 & 2 & 6 & 258 & 21.15 & 0.08 & 2 & 1 & & 4 & 84 & 258 & 20.69 & -0.01 & 2 & 3 & & 4 \\
\hline 50 & 260 & 20.40 & 0.06 & 2 & 3 & 4 & 2 & 69 & 260 & 21.34 & -0.01 & 2 & 1 & 3 & 4 & 107 & 261 & 20.57 & 0.04 & 2 & 2 & & 4 \\
\hline 38 & 262 & 21.39 & 0.08 & 2 & 0 & 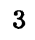 & 3 & 32 & 266 & 21.11 & 0.09 & 2 & 0 & & 2 & 69 & 267 & 19.55 & 0.01 & 2 & 0 & 4 & 1 \\
\hline 79 & 268 & 21.21 & 0.13 & 2 & 3 & 3 & 1 & 22 & 270 & 21.31 & 0.15 & 2 & 1 & 3 & 4 & 122 & 270 & 21.20 & -0.09 & 2 & 3 & & 6 \\
\hline 52 & 271 & 20.30 & 0.05 & 2 & 1 & 2 & 2 & 5 & 273 & 20.00 & 0.03 & & 1 & 2 & 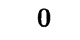 & 104 & 274 & 21.95 & 0.15 & 2 & 2 & & 6 \\
\hline 70 & 276 & 21.59 & 0.06 & 2 & 4 & 3 & 2 & 110 & 276 & 21.66 & 0.13 & 2 & 4 & 3 & 5 & 49 & 277 & 20.43 & -0.02 & 2 & 2 & $\mathbf{T}$ & 3 \\
\hline 59 & 280 & 20.60 & 0.00 & 2 & 1 & 4 & 3 & 100 & 282 & 20.63 & 0.01 & & 3 & & 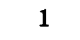 & 10 & 284 & 21.20 & 0.19 & 2 & $\sigma$ & & 7 \\
\hline 65 & 288 & 21.46 & 0.37 & 1 & 0 & 0 & 11 & 76 & 291 & 21.97 & 0.46 & 2 & 2 & 3 & 12 & 113 & 291 & 19.07 & 0.13 & 2 & 1 & 4 & 3 \\
\hline 19 & 292 & 19.94 & 0.05 & 2 & 0 & 4 & 4 & 64 & 295 & 20.92 & -0.01 & 2 & 1 & & 5 & 90 & 295 & 21.77 & 0.12 & 2 & , & & 0 \\
\hline 56 & 296 & 21.11 & 0.13 & 2 & 1 & 0 & 0 & 42 & 300 & 21.77 & 0.30 & 2 & 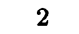 & & 14 & 77 & 301 & 20.87 & 0.02 & 2 & 1 & 3 & 0 \\
\hline 75 & 307 & 20.48 & 0.02 & 2 & 1 & 4 & 1 & 23 & 308 & 20.80 & 0.21 & 2 & 2 & 3 & 2 & 138 & 312 & 21.39 & 0.10 & 1 & 0 & 2 & 0 \\
\hline
\end{tabular}


TABLE III. (continued)

\begin{tabular}{|c|c|c|c|c|c|c|c|c|c|c|c|c|c|c|c|c|c|c|c|c|c|c|c|}
\hline & ix) & $r$ & g-r & \# & $\begin{array}{c}\sigma(r) \\
(\mathrm{mag})\end{array}$ & $\#$ & $\begin{array}{c}\sigma(g) \\
(\mathrm{mag})\end{array}$ & & $x^{y}$ & $\mathrm{r}$ & g-r & \# & $\begin{array}{c}\sigma(r) \\
(\mathrm{mag})\end{array}$ & \# & $\begin{array}{c}\sigma(g) \\
(\mathrm{mag})\end{array}$ & & $i x)^{y}$ & $\mathbf{r}$ & $g-r$ & $\#$ & $\begin{array}{c}\sigma(r) \\
(\mathrm{mag})\end{array}$ & $\#$ & $\begin{array}{c}\sigma(g) \\
(\mathrm{mag})\end{array}$ \\
\hline 49 & 314 & 20.55 & 0.02 & 2 & 1 & 4 & 10 & 106 & 318 & 21.17 & 0.13 & 2 & 2 & 3 & 2 & 75 & 320 & 21.60 & -0.37 & 2 & 48 & 3 & 3 \\
\hline 118 & 321 & 21.87 & 0.12 & 2 & 0 & 3 & 7 & 13 & 322 & 20.32 & 0.10 & 2 & 2 & 4 & 5 & 119 & 329 & 21.09 & 0.17 & 2 & 1 & 3 & 6 \\
\hline 123 & 330 & 21.18 & 0.10 & 2 & 4 & 3 & 7 & 92 & 333 & 21.40 & 0.19 & 2 & 1 & 3 & 6 & 9 & 334 & 20.85 & 0.25 & 2 & 1 & 3 & 7 \\
\hline 85 & 334 & 21.37 & 0.43 & 2 & 13 & 3 & 15 & 54 & 335 & 21.91 & 0.07 & 2 & 1 & 3 & 16 & 127 & 335 & 20.30 & -0.05 & 2 & 1 & 4 & 4 \\
\hline 94 & 339 & 21.50 & 0.23 & 2 & 2 & 3 & 3 & 26 & 340 & 19.97 & 0.02 & 2 & 2 & 4 & 6 & 75 & 340 & 21.28 & 0.21 & 2 & 1 & 3 & 10 \\
\hline 37 & 341 & 21.29 & 0.26 & 2 & 4 & 3 & 21 & 15 & 343 & 20.16 & 0.07 & 2 & 1 & 4 & 5 & 6 & 346 & 20.34 & -0.02 & 2 & 0 & 2 & 3 \\
\hline 121 & 346 & 21.85 & 0.27 & 2 & 4 & 3 & 4 & 64 & 347 & 20.18 & 0.03 & 2 & 0 & 4 & 1 & 88 & 350 & 21.46 & 0.03 & 1 & 0 & 2 & 1 \\
\hline 10 & 352 & 19.57 & 0.08 & 2 & 0 & 4 & 8 & 96 & 352 & 19.94 & 0.02 & 2 & 0 & 4 & 3 & 20 & 353 & 20.10 & -0.04 & 2 & 1 & 4 & 1 \\
\hline 85 & 355 & 20.08 & 0.01 & 2 & 1 & 4 & 4 & 59 & 356 & 19.44 & -0.02 & 2 & 2 & 4 & 2 & 68 & 357 & 18.93 & 0.18 & 2 & 0 & 4 & 2 \\
\hline 20 & 358 & 19.82 & 0.01 & 2 & 0 & 4 & 4 & 122 & 360 & 21.81 & 0.29 & 2 & 3 & 3 & 6 & 53 & 365 & 21.05 & 0.16 & 2 & 5 & 3 & 11 \\
\hline 11 & 366 & 20.14 & 0.06 & 2 & 0 & 4 & 5 & 103 & 366 & 20.33 & 0.09 & 2 & 2 & 4 & 6 & 31 & 367 & 20.65 & 0.08 & 2 & 3 & 4 & 7 \\
\hline 41 & 368 & 21.36 & 0.22 & 2 & 1 & 3 & 10 & 20 & 369 & 19.66 & 0.00 & 2 & 2 & 4 & 8 & 66 & 370 & 21.62 & 0.35 & 2 & 6 & 3 & 8 \\
\hline 91 & 370 & 20.94 & 0.12 & 2 & 3 & 3 & 4 & 124 & 370 & 22.00 & 0.15 & 2 & 10 & 0 & 4 & 131 & 370 & 21.23 & 0.17 & 1 & 0 & 3 & 2 \\
\hline 48 & 372 & 20.50 & 0.13 & 2 & 0 & 4 & 5 & 30 & 373 & 21.60 & 0.44 & 2 & 5 & 3 & 14 & 108 & 373 & 21.13 & 0.14 & 2 & 8 & 3 & 4 \\
\hline 39 & 375 & 21.66 & 0.30 & 2 & 7 & 3 & 6 & 84 & 376 & 19.54 & 0.03 & 2 & 1 & 4 & 4 & 115 & 377 & 20.48 & 0.02 & 2 & 0 & 1 & 1 \\
\hline 71 & 378 & 20.09 & 0.10 & 2 & 1 & 4 & 5 & 13 & 380 & 19.89 & 0.01 & 2 & 1 & 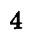 & 4 & 59 & 380 & 21.03 & 0.18 & 2 & 7 & 3 & 11 \\
\hline 124 & 380 & 20.02 & 0.01 & 2 & 1 & 4 & 4 & 34 & 382 & 20.24 & 0.05 & 2 & 1 & 4 & 4 & 67 & 385 & 21.25 & 0.13 & 2 & 10 & 2 & 0 \\
\hline 9 & 388 & 21.57 & 0.30 & 2 & 11 & 2 & 0 & 97 & 388 & 18.45 & 0.20 & 1 & 0 & 3 & 3 & 16 & 389 & 20.49 & 0.05 & 2 & 2 & 3 & 4 \\
\hline 73 & 389 & 20.53 & 0.10 & 2 & 4 & 3 & 6 & 38 & 391 & 20.18 & 0.06 & 2 & 1 & 3 & 5 & 76 & 394 & 21.60 & 0.52 & 1 & 0 & 2 & 1 \\
\hline & 394 & 20.29 & 0.04 & 2 & 3 & 3 & 3 & 122 & 395 & 21.07 & 0.10 & 2 & 6 & 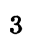 & 2 & & & & & & & & \\
\hline & Field & & & & & & & 23 & & 20.02 & 0.01 & 1 & 0 & 2 & 1 & 14 & 5 & 20.79 & 0.12 & 1 & 0 & 2 & 2 \\
\hline 116 & 5 & 20.02 & 0.04 & 2 & 3 & 4 & 1 & 45 & 6 & 18.93 & 0.19 & 2 & 2 & 4 & 2 & 88 & 6 & 21.30 & 0.22 & 2 & 7 & 3 & 4 \\
\hline 107 & 6 & 21.80 & 0.02 & 2 & 10 & 3 & 8 & 62 & 7 & 20.81 & 0.04 & 2 & 6 & 4 & 5 & 73 & 7 & 19.71 & 0.03 & 2 & 3 & 4 & 4 \\
\hline 131 & 8 & 20.56 & 0.09 & 2 & 6 & 4 & 3 & 26 & 9 & 19.81 & 0.04 & 2 & 2 & 4 & 2 & 92 & 11 & 21.18 & 0.18 & 2 & 8 & 3 & 5 \\
\hline 9 & 14 & 19.75 & -0.05 & 2 & 2 & 4 & 4 & 19 & 18 & 19.86 & 0.01 & 2 & 2 & 4 & 3 & 58 & 18 & 21.13 & 0.13 & 2 & 7 & 3 & 4 \\
\hline 79 & 18 & 19.06 & 0.46 & 2 & 1 & 4 & 49 & 127 & 20 & 21.04 & 0.09 & 2 & 4 & 3 & 2 & 5 & 21 & 21.12 & 0.04 & 2 & 4 & 3 & 12 \\
\hline 75 & 23 & 20.24 & -0.02 & 2 & 1 & 3 & 1 & 45 & 24 & 21.54 & 0.16 & 2 & 11 & 3 & 2 & 66 & 24 & 19.96 & 0.07 & 2 & 2 & 4 & 3 \\
\hline 89 & 26 & 21.84 & 0.24 & 2 & 5 & 3 & 1 & 115 & 26 & 19.21 & 0.07 & 2 & 2 & 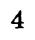 & 4 & 53 & 28 & 21.89 & 0.31 & 2 & 18 & 3 & 7 \\
\hline 21 & 29 & 21.95 & 0.27 & 2 & 15 & 2 & 2 & 135 & 30 & 21.77 & 0.20 & 2 & 17 & 3 & 15 & 77 & 31 & 21.14 & -0.80 & 2 & 4 & 2 & 2 \\
\hline 121 & 33 & 21.30 & 0.09 & 2 & 7 & 3 & 2 & 128 & 33 & 21.84 & 0.25 & 2 & 15 & 2 & 19 & 10 & 34 & 20.69 & -0.01 & 2 & 6 & 4 & 4 \\
\hline 39 & 34 & 20.21 & -0.03 & 2 & 4 & 4 & 3 & 87 & 34 & 20.62 & 0.05 & 2 & 3 & 4 & 0 & 49 & 35 & 20.95 & 0.12 & 2 & 7 & 4 & 4 \\
\hline 76 & 35 & 20.39 & 0.03 & 2 & 3 & 2 & 2 & 107 & 35 & 19.01 & -0.28 & 2 & 2 & 4 & 4 & 126 & 36 & 21.90 & 0.30 & 1 & 0 & 2 & 20 \\
\hline 4 & 37 & 19.68 & 0.02 & 2 & 2 & 4 & 4 & 65 & 38 & 19.45 & 0.07 & 2 & 1 & 4 & 2 & 53 & 39 & 20.21 & 0.06 & 2 & 4 & 4 & 3 \\
\hline 64 & 44 & 20.27 & 0.27 & 2 & 2 & 4 & 34 & 124 & 44 & 20.50 & 0.07 & 2 & 5 & 4 & 1 & 59 & 47 & 20.91 & 0.13 & 2 & 4 & 2 & 11 \\
\hline 109 & 47 & 20.15 & 0.01 & 2 & 4 & 4 & 1 & 10 & 51 & 21.33 & 0.20 & 2 & 7 & 3 & 11 & 116 & 56 & 21.86 & 0.29 & 2 & 8 & 3 & 14 \\
\hline 88 & 57 & 21.83 & 0.46 & 2 & 14 & 3 & 17 & 106 & 57 & 20.82 & 0.21 & 2 & 5 & 4 & 20 & 133 & 57 & 21.08 & 0.27 & 2 & 8 & 3 & 23 \\
\hline 10 & 58 & 21.96 & -0.80 & 2 & 16 & 3 & 58 & 66 & 58 & 21.97 & 0.10 & 1 & 0 & 2 & 9 & 96 & 60 & 20.77 & 0.08 & 2 & 3 & 4 & 8 \\
\hline 122 & 61 & 21.73 & 0.18 & 2 & 15 & 3 & 9 & 129 & 61 & 21.49 & 0.14 & 2 & 10 & 2 & 14 & 24 & 62 & 21.73 & 0.38 & 2 & 10 & 3 & 12 \\
\hline 7 & 64 & 20.74 & -0.29 & 2 & 31 & 3 & 4 & 12 & 64 & 21.22 & 0.13 & 1 & 0 & 2 & 9 & 58 & 64 & 19.92 & 0.00 & 2 & 4 & 4 & 5 \\
\hline 36 & 65 & 19.88 & 0.02 & 2 & 4 & 4 & 2 & 64 & 67 & 19.73 & 0.00 & 2 & 1 & 4 & 1 & 86 & 67 & 21.76 & 0.26 & 2 & 2 & 0 & 21 \\
\hline 48 & 69 & 20.00 & -0.02 & 2 & 3 & 4 & 2 & 132 & 70 & 21.64 & 0.16 & 2 & 12 & 0 & 6 & 84 & 72 & 21.51 & 0.22 & 2 & 8 & 2 & 4 \\
\hline 117 & 73 & 20.94 & 0.13 & 2 & 4 & 3 & 4 & 106 & 76 & 18.23 & 0.21 & 1 & 0 & 4 & 1 & 62 & 77 & 20.97 & 0.10 & 2 & 4 & 3 & 3 \\
\hline 27 & 79 & 17.48 & 0.24 & 1 & 0 & 3 & 3 & 25 & 87 & 19.98 & -0.06 & 2 & 3 & 4 & 6 & 126 & 88 & 21.54 & 0.04 & 1 & 0 & 3 & 2 \\
\hline 107 & 90 & 21.00 & 0.13 & 2 & 8 & 3 & 4 & 72 & 91 & 20.68 & 0.19 & 1 & 0 & 4 & 2 & 40 & 92 & 21.17 & 0.05 & 2 & 3 & 3 & 1 \\
\hline 52 & 92 & 20.10 & 0.00 & 2 & 2 & 4 & 1 & 64 & 94 & 20.89 & 0.15 & 2 & 1 & 3 & 13 & 36 & 97 & 21.04 & 0.17 & 2 & 0 & 2 & 2 \\
\hline 22 & 99 & 20.56 & -0.02 & 2 & 2 & 4 & 3 & 48 & 99 & 21.77 & 0.31 & 1 & 0 & 3 & 7 & 56 & 101 & 20.25 & -0.27 & 2 & 26 & 4 & 4 \\
\hline 97 & 101 & 21.77 & 0.07 & 2 & 20 & 3 & 5 & 123 & 102 & 19.99 & 0.01 & 2 & 1 & 4 & 1 & 103 & 103 & 20.60 & 0.07 & 2 & 3 & 4 & 2 \\
\hline 79 & 104 & 20.03 & 0.04 & 2 & 1 & 4 & 2 & 21 & 106 & 18.39 & 0.22 & 1 & 0 & 4 & 2 & 48 & 106 & 20.94 & 0.08 & 2 & 4 & 3 & 3 \\
\hline 29 & 108 & 20.34 & -0.08 & 2 & 1 & 4 & 1 & 40 & 110 & 20.12 & 0.05 & 2 & 1 & 4 & 1 & 100 & 111 & 21.99 & 0.19 & 2 & 11 & 3 & 9 \\
\hline
\end{tabular}


TABLE III. (continued)

\begin{tabular}{|c|c|c|c|c|c|c|c|c|c|c|c|c|c|c|c|c|c|c|c|c|c|c|c|}
\hline & ix) & $\mathrm{r}$ & g-r & $\#$ & $\begin{array}{c}\sigma(r) \\
(\mathrm{mag})\end{array}$ & $\#$ & $\begin{array}{c}\sigma(g) \\
(\mathrm{mag})\end{array}$ & & ix) & $\mathrm{r}$ & g-r & \# & $\begin{array}{c}\sigma(r) \\
(\mathrm{mag})\end{array}$ & $\#$ & $\begin{array}{c}\sigma(g) \\
(\mathrm{mag})\end{array}$ & & $x)^{y}$ & $\mathrm{r}$ & g-r & $\#$ & $\begin{array}{c}\sigma(r) \\
\text { (mag) }\end{array}$ & $\#$ & $\begin{array}{c}\sigma(g) \\
(\mathrm{mag})\end{array}$ \\
\hline 4 & 112 & 20.67 & 0.08 & 2 & 4 & 2 & 2 & 33 & 114 & 21.22 & 0.10 & 2 & 5 & 2 & 1 & 491 & 119 & 20.08 & 0.02 & 2 & 1 & 4 & 1 \\
\hline 22 & 121 & 20.70 & 0.05 & 2 & 0 & 4 & 2 & 14 & 123 & 18.37 & -0.09 & 1 & 0 & 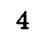 & 2 & 341 & 124 & 20.07 & -0.01 & 2 & 0 & 4 & 1 \\
\hline 55 & 127 & 19.24 & -1.08 & 2 & 122 & 3 & 1 & 89 & 127 & 21.58 & 0.27 & 2 & 6 & 2 & 0 & 127 & 127 & 19.52 & -0.03 & 2 & & 4 & 2 \\
\hline 23 & 131 & 19.55 & 0.02 & 2 & 1 & 4 & 1 & 132 & 132 & 21.10 & 0.04 & 2 & 6 & 3 & 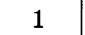 & 351 & 133 & 20.72 & 0.04 & 2 & & 4 & 1 \\
\hline 6 & 134 & 20.36 & 0.04 & 2 & 1 & 4 & 1 & 84 & 136 & 21.10 & 0.06 & 2 & 7 & 3 & 4 & 1131 & 136 & 20.67 & 0.03 & 2 & 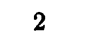 & 3 & 4 \\
\hline 39 & 137 & 20.72 & 0.02 & 2 & 5 & 4 & 2 & 54 & 137 & 19.86 & 0.50 & 2 & 1 & 4 & 79 & 131 & 140 & 20.78 & 0.03 & 2 & & $\mathbf{4}$ & 3 \\
\hline 49 & 142 & 21.42 & 0.38 & 2 & 2 & 3 & 13 & 89 & 142 & 20.29 & -0.43 & 2 & 2 & 3 & 44 & 114 & 142 & 19.86 & -0.12 & 2 & 1 & 4 & 3 \\
\hline 84 & 145 & 19.46 & -0.24 & 2 & 1 & 3 & 1 & 24 & 146 & 20.89 & 0.25 & 2 & 3 & 3 & 2 & 611 & 148 & 18.33 & 0.23 & 1 & & 4 & 1 \\
\hline 96 & 156 & 21.75 & 0.27 & 2 & 9 & 3 & 12 & 38 & 158 & 21.35 & 0.14 & 2 & 0 & 3 & 9 & 601 & 158 & 20.71 & 0.05 & 2 & & 4 & 2 \\
\hline 108 & 158 & 3 & 12 & 2 & 2 & 4 & 1 & 23 & 159 & 19.95 & 0.04 & 2 & & 4 & 2 & 122 & 159 & 24 & 0.27 & 2 & & 3 & 6 \\
\hline 39 & 164 & 21.48 & 0.12 & 2 & 7 & 2 & 1 & 73 & 166 & 21.22 & 0.14 & 2 & 0 & 3 & 4 & 911 & 166 & 21.14 & 0.10 & 2 & & 3 & 4 \\
\hline 34 & 167 & 21.37 & 0.25 & 2 & 6 & 3 & 5 & 107 & 167 & 20.42 & 0.02 & 2 & 3 & 4 & 3 & 971 & 177 & 19.82 & 0.01 & 2 & 2 & 4 & 3 \\
\hline 42 & 178 & .19 & -0.27 & 2 & 1 & 4 & 1 & 48 & 178 & 19.84 & -0.05 & 2 & 0 & 4 & 2 & 111 & 183 & 19.34 & 0.08 & 2 & & 4 & 1 \\
\hline 110 & 184 & 20.13 & 0.01 & 2 & 1 & 4 & 2 & 63 & 186 & 21.26 & 0.15 & 2 & 1 & 3 & 6 & 201 & 187 & 20.75 & 0.15 & 2 & 1 & 4 & 3 \\
\hline 31 & 189 & 2 & 13 & 2 & 3 & 3 & 1 & 51 & 189 & 21.76 & 0 & 2 & & 3 & 7 & 1341 & 189 & 21.52 & 0.14 & 1 & & 3 & 7 \\
\hline 88 & 194 & 20.51 & 0.06 & 2 & 1 & 4 & 4 & 118 & 194 & 19.27 & 0.10 & 2 & 1 & 4 & 1 & 128 & 195 & 21.27 & 0.07 & 2 & 4 & 3 & 1 \\
\hline 50 & 197 & 21.02 & 0.07 & 2 & 0 & 3 & 3 & 10 & 198 & 21.58 & 0.22 & 2 & 3 & 3 & 9 & 1012 & 200 & 21.76 & 0.26 & 2 & & 3 & 8 \\
\hline 115 & 201 & 20.96 & -0.01 & 2 & 4 & 4 & 4 & 1 & 202 & 20.04 & 0.02 & 1 & 0 & 3 & 0 & 402 & 204 & 21.38 & 0.37 & 2 & 3 & 3 & 34 \\
\hline 36 & 207 & 21.71 & -0.19 & 2 & 16 & 2 & 5 & 12 & 208 & 21.48 & 0.10 & 2 & 5 & 3 & 3 & 252 & 212 & 21.76 & 0.23 & 2 & 1 & 3 & 8 \\
\hline 52 & 214 & 20.92 & 0.80 & 2 & 1 & 3 & 54 & 106 & 214 & 21.21 & 0.15 & 2 & 3 & 3 & 4 & 1312 & 215 & 20.23 & 0.03 & 2 & & 4 & 1 \\
\hline 42 & 220 & 20.50 & 0.03 & 2 & 4 & 4 & 1 & 89 & 221 & 21.82 & 0.01 & 2 & 8 & 3 & 18 & 1292 & 224 & 20.87 & 0.23 & 2 & 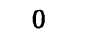 & 3 & 2 \\
\hline 85 & 225 & 21.51 & 0.20 & 2 & 4 & 2 & 13 & 18 & 226 & 19.35 & 0.10 & 2 & 2 & 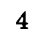 & ? & 292 & 227 & 20.22 & 0.02 & 2 & & 4 & 1 \\
\hline 2 & 237 & 20.82 & 0.06 & 2 & 0 & 3 & 4 & 32 & 240 & 19.82 & 0.01 & 2 & 0 & 4 & 0 & 1332 & 241 & 19.87 & 0.02 & 2 & 1 & 4 & 1 \\
\hline 75 & 242 & 20.83 & 0.05 & 2 & 1 & 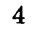 & 1 & 23 & 243 & 21.15 & 0.1 & 2 & 0 & 3 & 3 & 662 & 243 & 20.56 & 0.10 & 2 & & 4 & 4 \\
\hline 54 & 244 & 20.65 & 0.04 & 2 & 1 & 4 & 2 & 87 & 245 & 20.10 & 0.02 & 2 & 1 & 4 & 1 & 932 & 245 & 20.90 & -0.04 & 2 & 0 & 3 & 1 \\
\hline 115 & 245 & 18.20 & 21 & 1 & 0 & 4 & 2 & 104 & 246 & 20.00 & 0.02 & 2 & 1 & & 1 & 62 & 248 & 21.30 & 0.16 & 2 & 4 & 3 & 4 \\
\hline 15 & 249 & 20.83 & 00 & 2 & 0 & 4 & 2 & 0 & 250 & 21.03 & 0.04 & 2 & 1 & 3 & 3 & 482 & 255 & 21.28 & 0.22 & 2 & & 3 & 6 \\
\hline 124 & 256 & 20.88 & 13 & 2 & 3 & 3 & 4 & 71 & 261 & 20.62 & 0.15 & 2 & 1 & 4 & 4 & 822 & 261 & 20.28 & -0.01 & 2 & 4 & 4 & 4 \\
\hline 10 & 263 & 21.04 & 0.14 & 2 & 6 & 3 & 4 & 110 & 263 & 20.63 & 0.05 & 2 & 2 & 4 & 1 & 02 & 266 & 19.66 & -0.17 & 2 & & 3 & 5 \\
\hline 36 & 269 & 21.49 & 0.14 & 2 & 3 & 3 & 3 & 107 & 271 & 19.47 & -0.01 & 2 & 2 & 4 & 4 & 412 & 273 & 21.83 & 0.20 & 2 & 3 & 3 & 9 \\
\hline 33 & 275 & 21.97 & 0.46 & 2 & 0 & 3 & 11 & 119 & 278 & 20.65 & 0.07 & 2 & 2 & 4 & 1 & 72 & 280 & 20.98 & 0.06 & 2 & 1 & 3 & 2 \\
\hline 81 & 283 & 21.85 & 0.18 & 2 & 4 & 3 & 10 & 67 & 284 & 20.10 & 0.05 & 2 & 1 & 4 & 6 & 1172 & 285 & 21.81 & 0.19 & 2 & 4 & 3 & 2 \\
\hline 48 & 288 & 21.17 & ( & 2 & 4 & 2 & 5 & 8 & 292 & 19.77 & 0.01 & 2 & 3 & 4 & 2 & 532 & 292 & 20.58 & 0.08 & 2 & & 4 & 2 \\
\hline 56 & 299 & 20.66 & 0.06 & 2 & 2 & 4 & 5 & 86 & 300 & 21.56 & 0.14 & 2 & 1 & 3 & 2 & 213 & 301 & 21.70 & 0.24 & 2 & 0 & 3 & 1 \\
\hline 6 & 306 & 21.80 & 13 & 1 & 0 & 3 & 2 & 8 & 308 & 20.68 & 0 & 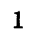 & 0 & 4 & 6 & 1253 & 310 & 20.93 & 0.12 & 2 & 2 & 3 & 1 \\
\hline 40 & 311 & 22.00 & 0.45 & 2 & 3 & 3 & 9 & 13 & 314 & 21.36 & 0.03 & 2 & 11 & 3 & 4 & 793 & 314 & 21.13 & 0.09 & 2 & 1 & 3 & 0 \\
\hline 31 & 323 & 20.45 & 0.00 & 2 & 3 & 4 & 2 & 102 & 334 & 21.20 & 0. & 2 & 1 & 3 & 2 & 403 & 335 & 21.55 & 0.23 & 2 & 1 & 2 & 0 \\
\hline 88 & 336 & 18.06 & 0.28 & 1 & 0 & 4 & 2 & 58 & 338 & 19.64 & 0.00 & 2 & 5 & $\mathbf{4}$ & 3 & 1063 & 339 & 20.98 & 0.17 & 2 & 3 & 3 & 0 \\
\hline 37 & 350 & 21.51 & 0.14 & 2 & 4 & 3 & 1 & 85 & 350 & 19.89 & 0.04 & 2 & 3 & 4 & 5 & 1333 & 351 & 18.32 & 0.41 & 1 & 0 & 4 & 25 \\
\hline 54 & 354 & 20.18 & 04 & 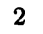 & 4 & 4 & 2 & 21 & 361 & 21.72 & 0.14 & 2 & 3 & 2 & 0 & 143 & 365 & 19.68 & -0.01 & 1 & 0 & 4 & 4 \\
\hline 28 & 365 & 21.05 & 0.04 & 2 & 3 & 3 & 5 & 43 & 367 & 19.56 & 0.06 & 2 & 5 & $A$ & 5 & 1163 & 367 & 20.73 & 0.01 & 2 & 1 & 4 & 3 \\
\hline 112 & 373 & 21.62 & 0.27 & 1 & 0 & 3 & 13 & 83 & 378 & 20.81 & 0.04 & 2 & 4 & 3 & 3 & 233 & 382 & 17.79 & 0.31 & 1 & 0 & 4 & 5 \\
\hline 11 & 384 & 20.44 & 0.04 & 2 & 3 & 3 & 1 & & & & & & & & & & & & & & & & \\
\hline & Field & & & & & & & 2 & 2 & 19.19 & -0.04 & 1 & 0 & 3 & 5 & 72 & 3 & 17 & 0.02 & 2 & 2 & 2 & 5 \\
\hline 112 & 5 & 20.40 & 0.02 & 2 & 1 & 4 & 5 & 96 & 6 & 20.46 & 0.03 & 2 & 3 & 4 & 4 & 90 & 8 & 21.37 & 0.05 & 2 & 1 & 3 & 7 \\
\hline 77 & 9 & 19.42 & -0.19 & 2 & 2 & 4 & 3 & 5 & 13 & 20.39 & -0.02 & 2 & 3 & 4 & 7 & 23 & 13 & 20.89 & -0.04 & 2 & 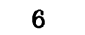 & 3 & 12 \\
\hline 93 & 14 & 21.09 & 0.02 & 2 & 2 & 3 & 5 & 50 & 16 & 20.34 & -0.02 & 2 & 8 & 4 & 4 & 38 & 17 & 19.90 & -0.03 & 2 & 21 & 4 & 22 \\
\hline 87 & 17 & 21.83 & -0.02 & 2 & 1 & 0 & $\gamma$ & 79 & 18 & 20.40 & -0.03 & 2 & 0 & 4 & 2 & 18 & 19 & 19.14 & 0.12 & 2 & 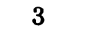 & 4 & 3 \\
\hline 98 & 19 & 21.22 & 0.06 & 2 & 1 & 3 & 9 & 61 & 21 & 20.64 & 0.05 & 2 & & 4 & & 8 & 22 & 20.10 & -0.02 & 2 & 6 & 4 & 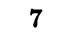 \\
\hline
\end{tabular}


TABLE III. (continued)

\begin{tabular}{|c|c|c|c|c|c|c|c|c|c|c|c|c|c|c|c|c|c|c|c|c|c|c|c|}
\hline & ix) & $\mathbf{r}$ & g-r & \# & $\begin{array}{c}\sigma(r) \\
(\mathrm{mag})\end{array}$ & \# & $\begin{array}{c}\sigma(g) \\
(\mathrm{mag})\end{array}$ & & ix) & $r$ & g-r & \# & $\begin{array}{c}\sigma(r) \\
(\mathrm{mag})\end{array}$ & \# & $\begin{array}{c}\sigma(g) \\
(\mathrm{mag})\end{array}$ & $\begin{array}{l}\mathbf{x} \\
\text { (pi }\end{array}$ & $y$ & $r$ & g-r & \# & $\begin{array}{c}\sigma(r) \\
(\mathrm{mag})\end{array}$ & \# & $\begin{array}{c}\sigma(g) \\
(\mathrm{mag})\end{array}$ \\
\hline 109 & 23 & 20.92 & -0.01 & 2 & 5 & 3 & 3 & 76 & 24 & 20.49 & 0.02 & 2 & 0 & 4 & 7 & 91 & 24 & 20.06 & -0.01 & 2 & 2 & 4 & 5 \\
\hline 102 & 26 & 19.50 & 0.03 & 2 & 1 & 4 & 5 & 61 & 29 & 19.18 & 0.10 & 2 & 3 & 4 & 4 & 23 & 32 & 20.81 & -0.10 & 2 & 29 & 3 & 24 \\
\hline 12 & 33 & 19.54 & -0.15 & 2 & 45 & 4 & 35 & 101 & 33 & 19.50 & 0.01 & 2 & 1 & 4 & 3 & 43 & 35 & 21.03 & 0.10 & 2 & 3 & 3 & 6 \\
\hline 82 & 35 & 20.39 & -0.02 & 2 & 4 & 4 & 9 & 108 & 35 & 20.50 & 0.02 & 2 & 3 & 4 & 8 & 25 & 36 & 20.60 & 0.06 & 1 & 0 & 2 & 4 \\
\hline 34 & 38 & 21.33 & 0.10 & 2 & 0 & 3 & 0 & 81 & 40 & 20.27 & 0.00 & 2 & 2 & 2 & 3 & 96 & 41 & 20.92 & 0.02 & 2 & 6 & 3 & 3 \\
\hline 114 & 42 & 16.87 & 0.28 & 1 & 0 & 3 & 3 & 24 & 43 & 20.32 & 0.02 & 2 & 0 & 4 & 5 & 12 & 46 & 20.23 & 0.00 & 2 & 3 & 4 & 6 \\
\hline 3 & 48 & 20.68 & 0.08 & 1 & 0 & 3 & 6 & 82 & 49 & 19.18 & 0.03 & 2 & 3 & 4 & 3 & 54 & 50 & 21.32 & -0.26 & 2 & 1 & 3 & 38 \\
\hline 91 & 52 & 21.15 & -0.01 & 2 & 1 & 2 & 0 & 109 & 53 & 19.99 & 0.25 & 2 & 7 & 4 & 7 & 39 & 55 & 20.11 & -0.01 & 2 & 2 & 4 & 5 \\
\hline 53 & 55 & 20.62 & -0.08 & 2 & 18 & 4 & 16 & 48 & 56 & 20.82 & -0.06 & 1 & 0 & 2 & 4 & 83 & 57 & 19.92 & -0.09 & 2 & 1 & 4 & 4 \\
\hline 10 & 58 & 20.41 & 0.01 & 2 & 1 & 4 & 10 & 72 & 58 & 21.77 & 0.18 & 1 & 0 & 3 & 12 & 65 & 59 & 21.74 & 0.25 & 2 & 4 & 3 & 2 \\
\hline 16 & 62 & 20.65 & 0.03 & 2 & 2 & 4 & 10 & 28 & 63 & 21.89 & 0.17 & 2 & 6 & 3 & 7 & 4 & 64 & 20.97 & -0.03 & 2 & 1 & 3 & 4 \\
\hline 104 & 65 & 19.38 & -0.21 & 2 & 1 & 4 & 5 & 113 & 65 & 20.40 & -0.01 & 2 & 4 & 3 & 5 & 10 & 68 & 21.39 & 0.18 & 2 & 7 & 3 & 7 \\
\hline 70 & 68 & 21.70 & 0.21 & 2 & 2 & 3 & 5 & 55 & 70 & 20.62 & 0.18 & 2 & 2 & 4 & 4 & 63 & 70 & 21.10 & 0.09 & 2 & 2 & 3 & 3 \\
\hline 88 & 70 & 20.18 & -0.01 & 2 & 2 & 4 & 5 & 18 & 71 & 21.36 & 0.17 & 2 & 3 & 2 & 0 & 36 & 72 & 20.00 & 0.05 & 2 & 2 & 4 & 2 \\
\hline 47 & 74 & 19.86 & 0.00 & 2 & 2 & 4 & 3 & 113 & 74 & 19.31 & 0.07 & 2 & 2 & 4 & 4 & 5 & 76 & 20.39 & -0.03 & 2 & 3 & 3 & 5 \\
\hline 27 & 81 & 21.58 & 0.00 & 1 & 0 & 2 & 4 & 51 & 82 & 20.68 & 0.09 & 2 & 1 & 4 & 6 & 63 & 82 & 21.08 & 0.02 & 2 & 1 & 3 & 4 \\
\hline 73 & 82 & 20.85 & 0.05 & 2 & 3 & 4 & 7 & 79 & 82 & 21.75 & -0.10 & 2 & 3 & 3 & 6 & 109 & 83 & 19.45 & 0.05 & 2 & 1 & 4 & 5 \\
\hline 18 & 85 & 20.92 & 0.05 & 2 & 6 & 3 & 6 & 36 & 85 & 20.16 & 0.01 & 2 & 0 & 4 & 3 & 26 & 87 & 20.97 & 0.03 & 2 & 7 & 2 & 9 \\
\hline 50 & 89 & 20.22 & 0.07 & 2 & 2 & 4 & 9 & 64 & 89 & 21.64 & 0.07 & 2 & 3 & 3 & 3 & 103 & 90 & 21.78 & 0.03 & 2 & 0 & 3 & 6 \\
\hline 6 & 91 & 21.22 & 0.04 & 2 & 4 & 3 & 5 & 28 & 93 & 19.93 & -0.07 & 2 & 5 & 3 & 2 & 94 & 96 & 21.92 & 0.14 & 2 & 0 & 2 & 3 \\
\hline 73 & 97 & 21.08 & 0.03 & 2 & 2 & 3 & 7 & 17 & 98 & 21.43 & 0.05 & 2 & 1 & 3 & 3 & 4 & 99 & 21.87 & 0.12 & 2 & 5 & 3 & 1 \\
\hline 64 & 100 & 19.59 & 0.04 & 2 & 3 & 4 & 4 & 103 & 103 & 21.47 & 0.02 & 1 & 0 & 0 & 4 & 72 & 106 & 21.68 & 0.13 & 2 & 0 & 3 & 5 \\
\hline 36 & 110 & 21.17 & 0.05 & 2 & 2 & 3 & 4 & 5 & 111 & 21.98 & 0.35 & 1 & 0 & 2 & 2 & 54 & 111 & 21.71 & -0.20 & 2 & 10 & 3 & 3 \\
\hline 20 & 115 & 20.99 & 0.00 & 2 & 2 & 3 & 4 & 50 & 115 & 21.81 & 0.10 & 2 & 4 & 3 & 3 & 78 & 115 & 20.90 & 0.04 & 2 & 1 & 3 & 2 \\
\hline 5 & 116 & 21.57 & 0.76 & 2 & 2 & 3 & 7 & 65 & 120 & 19.33 & -0.06 & 2 & 2 & 4 & 4 & 77 & 122 & 20.18 & -0.02 & 2 & 3 & 4 & 5 \\
\hline 9 & 123 & 21.62 & 0.13 & 2 & 5 & 3 & 5 & 0 & 126 & 21.43 & 0.12 & 1 & 0 & 2 & 4 & 35 & 126 & 20.92 & -0.02 & 2 & 2 & 3 & 2 \\
\hline 90 & 126 & 20.88 & 0.04 & 2 & 0 & 4 & 4 & 6 & 129 & 21.02 & 0.02 & 2 & 3 & 3 & 2 & 113 & 131 & 21.44 & 0.04 & 2 & 5 & 3 & 4 \\
\hline 5 & 134 & 21.26 & -0.02 & 2 & 2 & 3 & 3 & 95 & 135 & 20.64 & 0.00 & 2 & 0 & 4 & 7 & 78 & 140 & 19.65 & -0.03 & 2 & 4 & 4 & 6 \\
\hline 89 & 141 & 21.03 & 0.04 & 2 & 0 & 3 & 7 & 17 & 143 & 20.73 & 0.00 & 2 & 2 & 4 & 5 & 63 & 145 & 19.19 & 0.10 & 2 & 4 & 4 & 5 \\
\hline 34 & 148 & 19.57 & -0.03 & 2 & 3 & 4 & 1 & 87 & 148 & 21.25 & 0.00 & 2 & 1 & 3 & 7 & 82 & 151 & 21.02 & 0.01 & 2 & 3 & 3 & 4 \\
\hline 37 & 153 & 19.21 & 1.06 & 2 & 3 & 4 & 2 & 73 & 153 & 21.41 & 0.05 & 2 & 0 & 3 & 4 & -1 & 154 & 19.95 & -0.05 & 1 & 0 & 2 & 1 \\
\hline 88 & 157 & 21.39 & 0.02 & 2 & 0 & 3 & 3 & 24 & 159 & 21.89 & 0.22 & 2 & 3 & 3 & 6 & 17 & 167 & 21.76 & 0.01 & 2 & 0 & 2 & 0 \\
\hline 17 & 174 & 19.87 & -0.03 & 2 & 3 & 4 & 3 & 3 & 179 & 20.62 & 0.03 & 2 & 2 & 3 & 10 & 24 & 180 & 21.37 & -0.02 & 2 & 0 & 3 & 4 \\
\hline 48 & 180 & 19.42 & 0.02 & 2 & 4 & 4 & 3 & 57 & 181 & 21.26 & -0.70 & 2 & 64 & 2 & 1 & 31 & 183 & 19.97 & 0.19 & 2 & 3 & 4 & 40 \\
\hline 88 & 184 & 19.86 & 0.03 & 2 & 3 & 4 & 5 & 43 & 185 & 20.83 & -0.03 & 2 & 2 & 4 & 7 & 59 & 185 & 20.72 & 0.10 & 1 & 0 & 2 & 8 \\
\hline 101 & 185 & 21.87 & 0.08 & 2 & 0 & 3 & 5 & 26 & 188 & 20.70 & 0.07 & 2 & 9 & 3 & 1 & 39 & 188 & 20.49 & 0.00 & 2 & 4 & 4 & 5 \\
\hline 114 & 188 & 20.07 & -0.06 & 1 & 0 & 3 & 4 & 52 & 190 & 21.16 & 0.08 & 2 & 4 & 3 & 4 & 35 & 193 & 21.00 & -0.60 & 2 & 5 & 3 & 45 \\
\hline 52 & 195 & 21.24 & 0.10 & 2 & 3 & 2 & 3 & 4 & 198 & 20.54 & 0.05 & 1 & 0 & 3 & 4 & 39 & 198 & 20.27 & -0.04 & 2 & 3 & 2 & 1 \\
\hline 101 & 201 & 20.88 & 0.02 & 2 & 3 & 3 & 2 & 113 & 204 & 20.06 & 0.03 & 2 & 2 & 4 & 4 & 37 & 205 & 21.53 & 0.07 & 2 & 2 & 3 & 2 \\
\hline 65 & 205 & 21.91 & 0.13 & 2 & 4 & 3 & 1 & 86 & 205 & 20.37 & 0.13 & 2 & 3 & 4 & 21 & 4 & 211 & 20.88 & 0.34 & 2 & 2 & 3 & 2 \\
\hline 108 & 211 & 20.73 & 0.12 & 2 & 5 & 4 & 8 & 25 & 212 & 21.72 & -0.03 & 2 & 4 & 3 & 8 & 26 & 217 & 21.52 & 0.12 & 2 & 2 & 2 & 3 \\
\hline 85 & 217 & 18.04 & 0.22 & 1 & 0 & 4 & 1 & 101 & 217 & 19.24 & 0.12 & 2 & 2 & 4 & 4 & 58 & 218 & 21.16 & 0.02 & 2 & 2 & 3 & 1 \\
\hline 16 & 226 & 20.99 & 0.07 & 2 & 1 & 3 & 6 & 5 & 227 & 21.67 & -0.31 & 2 & 29 & 2 & 2 & 41 & 228 & 21.89 & 0.15 & 2 & 2 & 3 & 3 \\
\hline 0 & 232 & 20.35 & -0.04 & 1 & 0 & 2 & 1 & 61 & 235 & 21.44 & 0.00 & 2 & 2 & 3 & 2 & 11 & 238 & 21.31 & 0.12 & 2 & 1 & 3 & 1 \\
\hline 65 & 239 & 21.55 & 0.08 & 2 & 4 & 3 & 8 & 81 & 242 & 20.65 & -0.01 & 2 & 2 & 4 & 9 & 30 & 244 & 20.27 & -0.01 & 2 & 1 & 4 & 4 \\
\hline 2 & 246 & 21.23 & 0.13 & 1 & 0 & 2 & 2 & 76 & 246 & 19.71 & -0.01 & 2 & 0 & 4 & 3 & 35 & 248 & 20.99 & -0.01 & 2 & 0 & 4 & 6 \\
\hline 53 & 248 & 20.95 & -0.01 & 2 & 0 & 3 & 3 & 12 & 249 & 21.15 & 0.06 & 2 & 0 & 3 & 1 & 30 & 255 & 20.25 & -0.06 & 2 & 0 & 4 & 1 \\
\hline 17 & 262 & 19.89 & -0.05 & 2 & 0 & 4 & 1 & 64 & 266 & 21.57 & 0.42 & 2 & 1 & 0 & 2 & 54 & 268 & 20.42 & -0.02 & 2 & 0 & 4 & 3 \\
\hline 110 & 268 & 21.09 & -0.06 & 2 & 7 & 3 & 3 & 57 & 275 & 21.93 & 0.21 & 2 & 3 & 2 & 3 & 111 & 277 & 21.16 & 0.13 & 2 & 4 & 3 & 1 \\
\hline
\end{tabular}


TABLE III. (continued)

\begin{tabular}{|c|c|c|c|c|c|c|c|c|c|c|c|c|c|c|c|c|c|c|c|c|c|c|c|}
\hline & ix) & $\mathbf{r}$ & g-r & \# & $\begin{array}{c}\sigma(r) \\
(\mathrm{mag})\end{array}$ & \# & $\begin{array}{c}\sigma(g) \\
(\mathrm{mag})\end{array}$ & & $\mathrm{y}$ & $r$ & g-r & & $\begin{array}{c}\sigma(r) \\
(\mathrm{mag})\end{array}$ & $\#$ & $\begin{array}{c}\sigma(g) \\
(\mathrm{mag})\end{array}$ & & ix) & $\mathrm{r}$ & g-r & & $\begin{array}{c}\sigma(r) \\
(\mathrm{mag})\end{array}$ & & $\sigma(g)$ \\
\hline 97 & 278 & 20.68 & 09 & 2 & 4 & 4 & 4 & 32 & 286 & 21.86 & 0.31 & & 0 & 3 & 4 & 54 & 291 & 20.33 & -0.03 & 2 & - & & 2 \\
\hline 85 & 291 & 21.72 & 0.17 & 2 & 7 & J & 7 & 44 & 293 & 21.91 & 0.32 & & 0 & & 5 & 4 & 294 & 20.96 & 0.01 & 2 & 8 & & 4 \\
\hline 78 & 305 & 21.56 & 10 & 2 & 10 & 3 & 4 & 107 & 313 & 32 & 0.01 & & 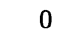 & & 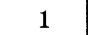 & 8 & 315 & 20.36 & 0.03 & 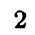 & & & 2 \\
\hline 19 & 320 & 21.52 & 0.37 & 2 & 6 & 3 & 13 & 28 & 323 & 21.63 & 0.01 & & 6 & & 9 & 59 & 323 & 20.14 & -0.02 & 2 & 5 & & 3 \\
\hline 100 & 332 & 21.92 & 0.23 & 2 & 7 & 3 & 10 & 61 & 334 & 21.29 & 0.12 & & 4 & & 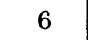 & 21 & 345 & 21.59 & 0.04 & 1 & 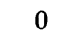 & & 3 \\
\hline & Field & & & & & & & 147 & & 19.35 & 0.01 & & 0 & 2 & 1 & 57 & & 22.00 & 0.20 & 2 & 4 & & 7 \\
\hline 68 & 6 & 21.94 & 0.27 & 2 & 1 & 3 & 5 & 120 & 6 & 17.76 & 0.20 & & 0 & 3 & 1 & 21 & 9 & 19.29 & 0.09 & 2 & 0 & 4 & 0 \\
\hline 91 & 10 & 21.95 & -0.15 & 1 & 0 & 3 & 22 & 81 & 14 & 20.11 & 0.04 & & 0 & 4 & 6 & 111 & 14 & 19.85 & 0.04 & 2 & 2 & & 3 \\
\hline 27 & 15 & 21.05 & 10 & 2 & 2 & 3 & 1 & 88 & 15 & 21.64 & 0.24 & & 5 & & 5 & 17 & 18 & 26 & 0.12 & 2 & 0 & & 1 \\
\hline 118 & 18 & 20.19 & 0.04 & 2 & 1 & 4 & 3 & 142 & 18 & 20.56 & 0.03 & & 0 & 4 & 4 & 54 & 19 & 00 & 0.05 & 2 & 2 & & 1 \\
\hline 42 & 22 & 19.04 & -0.03 & 2 & 0 & 4 & 2 & 80 & 22 & 21.93 & 0.13 & & 11 & & 9 & 20 & 24 & 21.93 & 0.14 & 2 & 1 & & 5 \\
\hline 101 & 24 & 20.99 & 0.12 & 2 & 2 & 3 & 2 & 75 & 25 & 21.78 & 0.22 & & 0 & 3 & 5 & 111 & 25 & 21.47 & 0.10 & 2 & 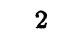 & & 6 \\
\hline 139 & 25 & 21.91 & 0.09 & 2 & 6 & 3 & 11 & 146 & 25 & 19.41 & 0.03 & & 0 & 3 & 1 & 119 & 26 & 20.77 & -0.01 & 2 & 4 & & 2 \\
\hline 124 & 28 & 19.35 & 06 & 2 & 0 & 4 & 3 & 56 & 34 & 20.01 & 0.01 & & 0 & $\mathbf{4}$ & 2 & 110 & 34 & 71 & 0.00 & 2 & 1 & & 1 \\
\hline 144 & 36 & 21.64 & 0.20 & 2 & 9 & 2 & 7 & 8 & 38 & 20.60 & 0.05 & 2 & 4 & 4 & 3 & 39 & 38 & 21.60 & 0.22 & 2 & 3 & & 0 \\
\hline 97 & 38 & 18.99 & 17 & 2 & 0 & 4 & 1 & 119 & 38 & 20.64 & 0.02 & & 2 & 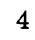 & 3 & 84 & 39 & 32 & 0.25 & 1 & 0 & & 1 \\
\hline 28 & 40 & 21.61 & 19 & 2 & 3 & 3 & 3 & 49 & 47 & 19.56 & -0.06 & 2 & 1 & 4 & 1 & 55 & 49 & 21.30 & 0.12 & 2 & 3 & & 2 \\
\hline 80 & 51 & 21.94 & 19 & 2 & 14 & 2 & 6 & 38 & 52 & 20.94 & 0.06 & & 0 & & 0 & 95 & 52 & 21.61 & 0.53 & 2 & 5 & & 23 \\
\hline 43 & 54 & 21.01 & 35 & 2 & 3 & 4 & 50 & 114 & 56 & 20.74 & 0.17 & 2 & 1 & 4 & 4 & 130 & 60 & 78 & 0.19 & 2 & 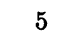 & & 3 \\
\hline 39 & 61 & 21.64 & 0.16 & 2 & 1 & 3 & 1 & 89 & 63 & 21.78 & 0.22 & $?$ & 0 & 3 & 6 & 123 & 65 & 53 & 0.13 & 2 & 3 & & 3 \\
\hline 98 & 66 & 6 & 18 & 2 & 4 & 3 & 1 & 118 & 66 & 3 & 24 & & & & $\epsilon$ & 62 & 69 & 34 & 05 & 2 & & & 1 \\
\hline 7 & 72 & 20.19 & -0.08 & 2 & 1 & 4 & 2 & 115 & 73 & 19.23 & 0.09 & & 0 & 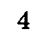 & 1 & 140 & 77 & 63 & 0.21 & 2 & 2 & & 2 \\
\hline 101 & 82 & 21.31 & 0.07 & 2 & 3 & 3 & 1 & 75 & 83 & 21.39 & 0.17 & & 2 & & 0 & 143 & 84 & 53 & 0.07 & 2 & 2 & & 4 \\
\hline 95 & 88 & 20.28 & 0.01 & 2 & 0 & 4 & 3 & 101 & 89 & 21.63 & 0.08 & 2 & 3 & 2 & 5 & 141 & 91 & 21.91 & 0.24 & 2 & 4 & 2 & 3 \\
\hline 81 & 95 & 20.96 & 07 & 2 & 3 & 3 & 1 & 103 & 95 & 21.64 & 0.16 & & 1 & & 4 & 43 & 99 & 21.27 & 0.13 & 2 & ( & & 1 \\
\hline 133 & 99 & 20.74 & 0.03 & 2 & 1 & 4 & 2 & 142 & 103 & 20.38 & 0.02 & 2 & 0 & 4 & 1 & 24 & 104 & 21.56 & 0.24 & 2 & & & 4 \\
\hline 63 & 105 & 21.35 & 0.44 & 2 & 1 & ? & 20 & 103 & 105 & 21.20 & -0.06 & 2 & 4 & & 14 & 119 & 105 & 20.86 & -2.29 & 2 & 4 & & 1 \\
\hline 18 & 106 & 21.20 & 0.07 & 2 & 1 & 3 & 1 & 41 & 109 & 20.20 & 0.00 & & 0 & & 2 & 121 & 110 & 18.88 & 0.14 & 2 & ( & & 1 \\
\hline 33 & 111 & 21.23 & 0.15 & 2 & 0 & 3 & 1 & 147 & 112 & 20.94 & 0.05 & 1 & 0 & 2 & 1 & 69 & 113 & 20.90 & 0.06 & 2 & 1 & 3 & 1 \\
\hline 101 & 114 & 18.14 & 0.22 & 1 & 0 & 4 & 2 & 134 & 114 & 19.70 & 0.03 & & c & & 1 & 43 & 115 & 19.63 & 0.01 & 2 & . & & 2 \\
\hline 115 & 115 & 20.17 & -0.09 & 2 & 7 & 2 & 5 & 77 & 116 & 20.60 & 0.05 & 2 & 2 & 4 & 6 & 17 & 118 & 20.95 & 0.05 & 2 & 1 & 3 & 1 \\
\hline 44 & 120 & 20.98 & 4 & 2 & 7 & 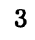 & 3 & 52 & 122 & 20.09 & 0 & & 2 & & 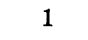 & 68 & 122 & 62 & 0.04 & 2 & 4 & & 3 \\
\hline 117 & 123 & 17.82 & 20 & 1 & 0 & 3 & 1 & 90 & 129 & 21.04 & 0.23 & 2 & 5 & 3 & 2 & 64 & 130 & 20.13 & -0.01 & 2 & ( & 4 & 2 \\
\hline 12 & 131 & 21.30 & 0.13 & 2 & 1 & 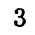 & 3 & 146 & 131 & 21.34 & 0.12 & & 0 & & 2 & 47 & 132 & 05 & 0.08 & 2 & 2 & & 2 \\
\hline 131 & 138 & 20.72 & 0.72 & 2 & 2 & 3 & 1 & 139 & 138 & 21.23 & 0.05 & 2 & 3 & 3 & 2 & 117 & 139 & 21.73 & 0.23 & 2 & 0 & 3 & 10 \\
\hline 40 & 150 & 21.36 & 12 & 2 & 5 & 3 & 1 & 59 & 153 & 21.77 & 0.15 & 2 & 3 & & 3 & 147 & 156 & 20.89 & 0.01 & 1 & 0 & 2 & 0 \\
\hline 43 & 157 & 20.70 & 06 & 2 & 2 & 4 & 2 & 9 & 158 & 21.71 & 0.17 & & 2 & & 3 & 28 & 165 & 20.49 & 0.05 & 2 & c & & 7 \\
\hline 34 & 168 & 20.58 & 0.03 & 2 & 0 & 4 & 1 & 90 & 171 & 21.67 & 0.07 & 2 & 0 & & 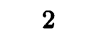 & 137 & 171 & 23 & 0.13 & 2 & & 4 & 2 \\
\hline 117 & 173 & 20.21 & ( & 2 & 2 & 4 & 1 & 82 & 174 & 21.12 & 0.27 & & 2 & & 1 & 91 & 178 & & 0.07 & 2 & & & 13 \\
\hline 18 & 179 & 20.36 & 0.01 & 2 & 1 & 4 & 2 & 105 & 181 & 21.33 & -0.01 & 2 & 1 & & 2 & 9 & 182 & 20.51 & -0.02 & 2 & & 4 & 1 \\
\hline 67 & 184 & 4 & 10 & 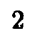 & 1 & 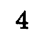 & 23 & 93 & 184 & 21.68 & 0.17 & & 0 & & 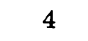 & 146 & 184 & 20.05 & 0.00 & 1 & & 2 & 1 \\
\hline 104 & 190 & 21.12 & 06 & 2 & 4 & 3 & 0 & 119 & 190 & 21.45 & 0.17 & 2 & 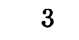 & & 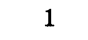 & 21 & 201 & 20.78 & 0.02 & 2 & 1 & 4 & 1 \\
\hline 111 & 204 & 21.03 & 0.12 & 2 & 3 & 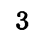 & 1 & 133 & 212 & 21.47 & 0.17 & 2 & 5 & & 5 & 125 & 221 & 20.96 & 0.08 & 2 & & 3 & 1 \\
\hline 50 & 224 & 18.78 & 0.20 & 2 & 1 & 4 & 2 & 139 & 226 & 20.34 & 1.22 & 2 & 1 & & 4 & 21 & 233 & 21.03 & 0.12 & 1 & 0 & 2 & 0 \\
\hline 48 & 233 & 20.87 & 0.30 & 2 & 1 & 4 & 40 & 77 & 234 & 21.84 & 0.17 & 2 & 10 & & $\pi$ & 23 & 241 & 19.60 & 0.17 & 2 & & 4 & 3 \\
\hline 96 & 241 & 20.70 & 0.05 & 2 & 0 & 4 & 3 & 80 & 243 & 21.96 & 0.22 & 2 & $y$ & 3 & 4 & 107 & 244 & 20.15 & 0.04 & 2 & 1 & 4 & 0 \\
\hline 48 & 246 & 21.52 & 0.12 & 2 & 3 & 0 & 0 & 58 & 249 & 19.99 & 0.03 & & 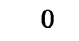 & & 4 & 69 & 249 & 20.29 & 0.03 & 2 & & 4 & 4 \\
\hline 43 & 250 & 20.31 & 0.05 & 2 & 1 & 4 & 0 & 61 & 256 & 21.36 & 0.10 & 2 & 2 & 3 & 2 & 116 & 256 & 21.06 & 0.12 & 2 & 4 & 3 & 3 \\
\hline 0 & 261 & 19.84 & 0.01 & 2 & 1 & 4 & 1 & 17 & 265 & 21.14 & 0.16 & & 3 & & 2 & 83 & 269 & 18.16 & 0.23 & 1 & & & 2 \\
\hline
\end{tabular}


TABLE IV. Magnitudes of NGC 6229 stars.

\begin{tabular}{|c|c|c|c|c|c|c|c|c|c|c|c|c|c|c|c|c|c|c|c|c|c|c|c|}
\hline & ix) & $\mathbf{r}$ & g-r & \# & $\begin{array}{c}\sigma(r) \\
(\mathrm{mag})\end{array}$ & $\#$ & $\begin{array}{c}\sigma(g) \\
(\mathrm{mag})\end{array}$ & $\begin{array}{l}\mathbf{x} \\
(\mathrm{pi}\end{array}$ & & $\mathbf{r}$ & g-r & $\#$ & $\begin{array}{c}\sigma(r) \\
(\mathrm{mag})\end{array}$ & \# & $\begin{array}{c}\sigma(g) \\
(\mathrm{mag})\end{array} \mid$ & $\begin{array}{l}\mathbf{x} \\
(\mathrm{pi}\end{array}$ & ix) & $r$ & g-r & \# & $\begin{array}{c}\sigma(r) \\
(\mathrm{mag})\end{array}$ & \# & $\begin{array}{c}\sigma(g) \\
(\mathrm{mag})\end{array}$ \\
\hline & Field & & & & & & & 65 & 8 & 22.25 & -0.15 & 2 & 4 & 4 & 13 & 134 & 18 & 22.23 & -0.10 & 3 & 2 & 5 & 9 \\
\hline 173 & 23 & 20.73 & 0.05 & 3 & 2 & 5 & 8 & 127 & 25 & 21.39 & 0.13 & 3 & 3 & 5 & 11 & 12 & 46 & 22.91 & 0.09 & 3 & 8 & 4 & 19 \\
\hline 75 & 48 & 22.21 & -0.48 & 3 & 65 & 5 & 10 & 85 & 52 & 22.52 & 0.09 & 3 & 4 & 4 & 7 & 109 & 53 & 22.84 & -0.13 & 3 & 1 & 4 & 14 \\
\hline 145 & 53 & 21.98 & 0.02 & 3 & 1 & 5 & 10 & 166 & 56 & 20.27 & -0.10 & 3 & 4 & 5 & 7 & 118 & 57 & 23.18 & 0.00 & 3 & 4 & 4 & 13 \\
\hline 21 & 58 & 20.64 & 0.99 & 3 & 4 & 5 & 16 & 106 & 60 & 22.29 & -0.09 & 3 & 2 & 4 & 7 & 193 & 61 & 22.40 & 0.03 & 2 & 6 & 4 & 7 \\
\hline 58 & 70 & 22.87 & -0.02 & 3 & 17 & 4 & 13 & 175 & 74 & 22.00 & -0.20 & 3 & 49 & 4 & 58 & 184 & 74 & 23.04 & 0.09 & 3 & 9 & 4 & 16 \\
\hline 191 & 75 & 22.57 & 0.00 & 3 & 3 & 4 & 9 & 125 & 80 & 22.29 & 0.00 & 3 & 6 & 4 & 9 & 9 & 81 & 23.41 & -0.43 & 3 & 32 & 3 & 5 \\
\hline 50 & 81 & 22.33 & 0.18 & 3 & 4 & 4 & 23 & 71 & 85 & 22.84 & -0.85 & 3 & 108 & 5 & 9 & 63 & 87 & 20.82 & 0.10 & 3 & 2 & 5 & 9 \\
\hline 132 & 87 & 22.12 & 0.00 & 3 & 1 & 5 & 13 & 150 & 87 & 22.92 & -0.01 & 3 & 8 & 4 & 7 & 105 & 88 & 22.77 & 0.04 & 3 & 57 & 4 & 74 \\
\hline 84 & 91 & 22.41 & -0.01 & 3 & 11 & 4 & 9 & 71 & 96 & 20.16 & 0.21 & 3 & 4 & 5 & 7 & 58 & 97 & 22.29 & 0.15 & 3 & 6 & 4 & 7 \\
\hline 140 & 97 & 23.06 & 0.09 & 3 & 3 & 3 & 18 & 120 & 99 & 22.80 & 0.06 & 3 & 2 & 4 & 15 & 40 & 100 & 22.44 & 0.03 & 3 & 3 & 4 & 9 \\
\hline 84 & 101 & 22.15 & 0.12 & 3 & 4 & 4 & 8 & 64 & 102 & 22.50 & 0.72 & 3 & 3 & 4 & 18 & 111 & 103 & 23.04 & -0.01 & 3 & 16 & 4 & 8 \\
\hline 54 & 107 & 22.56 & -0.01 & 3 & 34 & 4 & 28 & 115 & 110 & 22.59 & 0.40 & 3 & 9 & 3 & 39 & 49 & 118 & 22.67 & -0.61 & 3 & 57 & 4 & 11 \\
\hline 55 & 119 & 22.01 & -0.02 & 2 & 4 & 3 & 6 & 123 & 119 & 22.46 & 0.04 & 3 & 3 & 4 & 5 & 8 & 121 & 21.70 & 0.02 & 3 & 3 & 5 & 15 \\
\hline 67 & 122 & 21.52 & -0.12 & 3 & 12 & 4 & 5 & 153 & 124 & 22.42 & 0.09 & 3 & 8 & 4 & 3 & 80 & 125 & 21.38 & 0.03 & 3 & 2 & 5 & 8 \\
\hline 142 & 125 & 23.05 & 0.10 & 3 & 12 & 4 & 7 & 13 & 127 & 21.76 & 0.03 & 3 & 2 & 5 & 7 & 122 & 128 & 22.87 & 0.17 & 3 & 24 & 4 & 5 \\
\hline 145 & 135 & 22.25 & -0.01 & 3 & 5 & 4 & 4 & 57 & 138 & 21.11 & 0.02 & 3 & 4 & 5 & 8 & 116 & 138 & 21.82 & -0.05 & 3 & 8 & 5 & 4 \\
\hline 151 & 138 & 23.04 & 0.12 & 3 & 4 & 3 & 6 & 67 & 140 & 21.52 & -0.70 & 3 & 117 & 5 & 9 & 86 & 141 & 22.31 & -0.26 & 3 & 21 & 5 & 5 \\
\hline 180 & 141 & 23.14 & 0.09 & 3 & 33 & 3 & 16 & 16 & 142 & 22.95 & 0.07 & 3 & 21 & 4 & 15 & 77 & 142 & 21.85 & -0.34 & 3 & 53 & 5 & 4 \\
\hline 48 & 144 & 22.30 & 0.13 & 3 & 1 & 3 & 2 & 135 & 145 & 22.67 & 0.06 & 2 & 11 & 4 & 17 & 117 & 149 & 20.64 & 0.15 & 3 & 3 & 5 & 5 \\
\hline 155 & 150 & 22.83 & 0.15 & 3 & 8 & 4 & 7 & 81 & 151 & 21.11 & 0.03 & 3 & 7 & 5 & 4 & 74 & 152 & 22.98 & 0.01 & 3 & 18 & 3 & 6 \\
\hline 35 & 153 & 21.37 & 0.07 & 3 & 1 & 5 & 10 & 131 & 153 & 23.14 & -0.03 & 3 & 7 & 3 & 12 & 85 & 155 & 20.35 & 0.22 & 3 & 9 & 3 & 4 \\
\hline 58 & 157 & 20.75 & 0.13 & 3 & 3 & 4 & 5 & 173 & 157 & 22.78 & 0.05 & 3 & 4 & 4 & 16 & 190 & 157 & 21.13 & 0.00 & 3 & 2 & 5 & 7 \\
\hline 26 & 158 & 20.94 & 0.04 & 3 & 13 & 5 & 10 & 116 & 159 & 20.48 & 0.17 & 3 & 2 & 5 & 6 & 81 & 161 & 21.73 & 0.09 & 3 & 7 & 5 & 11 \\
\hline 134 & 161 & 21.75 & -0.01 & 3 & 4 & 5 & 8 & 167 & 163 & 21.80 & 0.09 & 3 & 67 & 4 & 51 & 50 & 165 & 21.70 & 0.03 & 2 & 3 & 5 & 7 \\
\hline 77 & 165 & 21.71 & 0.06 & 2 & 3 & 5 & 9 & 172 & 165 & 20.91 & 0.00 & 2 & 3 & 4 & 5 & 94 & 168 & 23.04 & -0.07 & 2 & 16 & 3 & 3 \\
\hline 22 & 171 & 22.34 & 0.10 & 3 & 4 & 4 & 10 & 106 & 171 & 22.95 & -0.52 & 3 & 2 & 5 & 45 & 34 & 172 & 23.01 & 0.23 & 3 & 7 & 3 & 11 \\
\hline 61 & 172 & 21.99 & 0.20 & 3 & 61 & 3 & 78 & 138 & 173 & 23.24 & -0.17 & 2 & 11 & 3 & 6 & 163 & 175 & 22.55 & 0.27 & 3 & 38 & 3 & 37 \\
\hline 84 & 176 & 21.72 & 0.00 & 3 & 6 & 5 & 7 & 106 & 176 & 23.50 & -0.24 & 2 & 7 & 3 & 17 & 127 & 177 & 21.53 & 0.01 & 3 & 1 & 5 & 8 \\
\hline 4 & 181 & 21.18 & -0.02 & 2 & 5 & 3 & 4 & 78 & 181 & 21.86 & -0.54 & 3 & 81 & 5 & 4 & 46 & 182 & 22.56 & -1.08 & 3 & 19 & 5 & 58 \\
\hline 157 & 183 & 22.92 & 0.07 & 3 & 3 & 3 & 11 & 104 & 186 & 23.07 & -0.70 & 3 & 41 & 4 & 10 & 112 & 186 & 22.60 & -0.34 & 3 & 37 & 4 & 4 \\
\hline 116 & 188 & 22.56 & 0.29 & 3 & 3 & 3 & 10 & 17 & 189 & 21.56 & 0.00 & 3 & 6 & 5 & 8 & 165 & 189 & 21.84 & 0.06 & 3 & 7 & 5 & 4 \\
\hline 40 & 190 & 22.79 & 0.10 & 3 & 12 & 4 & 3 & 61 & 190 & 21.24 & 0.12 & 3 & 8 & 5 & 6 & 116 & 191 & 22.46 & 0.17 & 3 & 10 & 3 & 12 \\
\hline 150 & 191 & 20.63 & 0.19 & 2 & 3 & 5 & 10 & 177 & 191 & 22.20 & -0.35 & 3 & 52 & 5 & 5 & 24 & 195 & 21.55 & 0.08 & 3 & 4 & 5 & 8 \\
\hline 76 & 195 & 22.06 & 0.24 & 3 & 16 & 4 & 7 & 86 & 195 & 22.56 & 0.15 & 3 & 9 & 4 & 3 & 94 & 195 & 22.14 & 0.19 & 3 & 34 & 3 & 5 \\
\hline 99 & 195 & 21.72 & 0.01 & 2 & 1 & 5 & 11 & 160 & 195 & 21.57 & -0.04 & 3 & 1 & 5 & 6 & 168 & 196 & 22.78 & 0.07 & 3 & 22 & 4 & 18 \\
\hline & Field & & & & & & & 166 & 34 & 23.05 & -0.67 & 3 & 93 & 3 & 5 & 146 & 36 & 22.49 & 0.06 & 3 & 1 & 3 & 2 \\
\hline 100 & 45 & 22.31 & 0.09 & 3 & 4 & 3 & 3 & 144 & 48 & 21.49 & 0.00 & 3 & 2 & 3 & 4 & 161 & 50 & 21.70 & -0.01 & 3 & 1 & 3 & 3 \\
\hline 10 & 51 & 22.65 & -0.14 & 3 & 10 & 3 & 16 & 126 & 51 & 21.82 & -0.01 & 3 & 3 & 3 & 3 & 119 & 53 & 22.92 & 0.00 & 3 & 10 & 3 & 7 \\
\hline 187 & 53 & 21.83 & 0.04 & 3 & 3 & 3 & 5 & 133 & 59 & 22.62 & 0.57 & 3 & 5 & 3 & 5 & 173 & 60 & 22.24 & 0.02 & 3 & 5 & 3 & 4 \\
\hline 98 & 65 & 19.46 & 0.20 & 3 & 2 & 3 & 4 & 149 & 70 & 23.11 & -0.25 & 2 & 30 & 3 & 3 & 138 & 72 & 22.29 & 0.02 & 3 & 1 & 3 & 3 \\
\hline 188 & 77 & 22.88 & 0.21 & 3 & 9 & 3 & 4 & 56 & 83 & 22.80 & 0.09 & 3 & 3 & 3 & 9 & 145 & 84 & 22.10 & -1.15 & 3 & 81 & 3 & 4 \\
\hline 153 & 96 & 22.43 & 0.10 & 2 & 6 & 3 & 11 & 135 & 108 & 22.43 & 0.07 & 2 & 1 & 3 & 4 & 164 & 114 & 21.82 & 0.09 & 3 & 2 & 3 & 8 \\
\hline 54 & 115 & 21.74 & -0.01 & 3 & 3 & 3 & 5 & 130 & 121 & 22.34 & 0.14 & 3 & 3 & 3 & 5 & 190 & 123 & 21.09 & 1.19 & 3 & 2 & 3 & 5 \\
\hline 70 & 124 & 22.34 & 0.03 & 3 & 4 & 3 & 5 & 148 & 126 & 21.37 & 0.04 & 3 & 1 & 3 & 2 & 59 & 127 & 22.97 & 0.08 & 3 & 7 & 3 & 4 \\
\hline 192 & 130 & 21.31 & 0.01 & 3 & 10 & 3 & 4 & 81 & 132 & 21.60 & 0.01 & 3 & 3 & 3 & 3 & 159 & 132 & 21.22 & 0.06 & 3 & 1 & 3 & 5 \\
\hline 173 & 139 & 22.15 & 0.05 & 3 & 5 & 3 & 4 & 35 & 141 & 22.04 & -0.01 & 3 & 5 & 3 & 4 & 110 & 141 & 21.81 & 0.04 & 3 & 2 & 3 & 1 \\
\hline 13 & 142 & 22.40 & 0.26 & 3 & 4 & 3 & 3 & 121 & 142 & 22.15 & 0.14 & 3 & 2 & 3 & 5 & 89 & 151 & 22.77 & 0.05 & 3 & 13 & 3 & 9 \\
\hline 144 & 153 & 22.42 & 0.15 & 3 & 5 & 3 & 4 & 128 & 155 & 22.06 & 0.06 & 3 & 5 & 3 & 2 & 80 & 157 & 22.30 & 0.08 & 3 & 5 & 3 & 1 \\
\hline
\end{tabular}


TABLE IV. (continued)

\begin{tabular}{|c|c|c|c|c|c|c|c|c|c|c|c|c|c|c|c|c|c|c|c|c|c|c|c|}
\hline & ix) & $\mathbf{r}$ & g-r & $\#$ & $\begin{array}{c}\sigma(r) \\
(\mathrm{mag})\end{array}$ & $\#$ & $\begin{array}{c}\sigma(g) \\
(\mathrm{mag})\end{array}$ & & ix) & $r$ & g-r & \# & $\begin{array}{c}\sigma(r) \\
(\mathrm{mag})\end{array}$ & $\#$ & $\begin{array}{c}\sigma(g) \\
(\mathrm{mag})\end{array}$ & & $\begin{array}{l}\mathbf{y} \\
\mathbf{x})\end{array}$ & $r$ & g-r & $\#$ & $\begin{array}{c}\sigma(r) \\
(\mathrm{mag})\end{array}$ & $\#$ & $\begin{array}{c}\sigma(g) \\
(\mathrm{mag})\end{array}$ \\
\hline 32 & 158 & 22.99 & 0.25 & 3 & 12 & 3 & 13 & 63 & 160 & 22.33 & 0.01 & 3 & 2 & 3 & 3 & 82 & 1632 & 22.75 & 0.14 & 3 & 6 & 3 & 5 \\
\hline 136 & 164 & 22.65 & 0.15 & 3 & 3 & 3 & 3 & 144 & 164 & 22.34 & 0.07 & 2 & 4 & 3 & 7 & 192 & 1662 & 22.50 & 0.23 & 3 & 4 & 3 & 1 \\
\hline 171 & 168 & 21.65 & 0.10 & 3 & 4 & 3 & 6 & 116 & 170 & 22.32 & 0.16 & 3 & 2 & 3 & 0 & 171 & 1732 & 22.05 & -0.02 & 3 & 9 & 3 & 5 \\
\hline 176 & 173 & 22.14 & 0.04 & 3 & 4 & 3 & 1 & 160 & 174 & 22.18 & 0.20 & 3 & 4 & 3 & 4 & 101 & 1772 & 21.76 & 0.08 & 3 & 0 & 3 & 6 \\
\hline 173 & 179 & 21.89 & 0.09 & 3 & 3 & 3 & 3 & 67 & 180 & 22.85 & -0.63 & 3 & 45 & 3 & 3 & 192 & 1802 & 22.79 & -0.30 & 3 & 65 & 3 & 8 \\
\hline 126 & 186 & 22.42 & 0.12 & 3 & 5 & 3 & 2 & 133 & 188 & 21.56 & -0.01 & 3 & 2 & 3 & 4 & 137 & 1932 & 21.08 & -0.06 & 2 & 0 & 3 & 5 \\
\hline 120 & 194 & 21.69 & 0.02 & 2 & 2 & 3 & 3 & 156 & 194 & 22.82 & 0.55 & 2 & 6 & 3 & 30 & 192 & 1952 & 21.82 & 0.14 & 3 & 6 & 3 & 4 \\
\hline & Field & & & & & & & 28 & & 20.72 & 0.21 & 2 & 6 & 3 & 4 & 99 & 52 & 22.55 & 0.05 & 2 & 5 & 4 & 3 \\
\hline 113 & 7 & 22.98 & -0.05 & 3 & 44 & 3 & 9 & 51 & 8 & 22.94 & 0.24 & 3 & 6 & 3 & 15 & 185 & 82 & 22.16 & 0.16 & 3 & 5 & 5 & 6 \\
\hline 195 & 8 & 21.37 & 0.17 & 3 & 8 & 5 & 7 & 174 & 9 & 20.97 & 0.13 & 3 & 10 & 5 & 9 & 126 & 112 & 22.97 & 0.41 & 2 & 4 & 3 & 5 \\
\hline 137 & 12 & 20.90 & 0.26 & 3 & 9 & 3 & 2 & 110 & 13 & 19.76 & 0.25 & 3 & 6 & 5 & 4 & 27 & 152 & 22.12 & 0.05 & 3 & 6 & 3 & 0 \\
\hline 131 & 19 & 21.33 & 0.29 & 3 & 8 & 4 & 3 & 191 & 19 & 20.62 & 0.14 & 3 & 6 & 5 & 3 & 52 & 202 & 23.13 & 0.27 & 3 & 15 & 3 & 9 \\
\hline 103 & 20 & 22.57 & 0.14 & 3 & 18 & 4 & 0 & 186 & 20 & 20.81 & 0.35 & 3 & 14 & 4 & 11 & 47 & 212 & 22.40 & 0.10 & 3 & 6 & 3 & 3 \\
\hline 158 & 21 & 22.18 & 0.35 & 3 & 13 & 4 & 9 & 97 & 22 & 22.72 & 0.18 & 3 & 6 & 4 & 3 & 71 & 242 & 22.33 & 0.10 & 3 & 6 & 5 & 2 \\
\hline 78 & 30 & 22.25 & 0.09 & 3 & 2 & 5 & 4 & 91 & 32 & 23.39 & 0.26 & 2 & 7 & 3 & 31 & 171 & 322 & 22.05 & 0.07 & 3 & 7 & 5 & 4 \\
\hline 131 & 33 & 21.32 & 0.12 & 3 & 7 & 5 & 4 & 150 & 33 & 22.34 & 0.42 & 3 & 9 & 4 & 10 & 184 & 352 & 22.36 & 0.15 & 2 & 9 & 4 & 7 \\
\hline 51 & 39 & 21.84 & 0.05 & 3 & 4 & 5 & 3 & 17 & 40 & 21.43 & -0.01 & 3 & 8 & 3 & 5 & 65 & 412 & 21.01 & 0.10 & 3 & 4 & 5 & 5 \\
\hline 78 & 41 & 21.93 & 0.08 & 3 & 4 & 5 & 4 & 180 & 43 & 22.81 & 0.16 & 3 & 5 & 4 & 3 & 100 & 462 & 22.73 & -0.09 & 3 & 14 & 4 & 2 \\
\hline 168 & 46 & 22.06 & 0.14 & 3 & 1 & 5 & 5 & 22 & 48 & 22.06 & 0.01 & 3 & 3 & 3 & 4 & 90 & 492 & 21.41 & 0.06 & 3 & 6 & 5 & 15 \\
\hline 100 & 50 & 22.84 & 0.15 & 2 & 2 & 4 & 10 & 148 & 50 & 23.24 & 0.21 & 2 & 18 & 3 & 3 & 121 & 512 & 22.04 & 0.12 & 3 & 6 & 4 & 21 \\
\hline 136 & 52 & 23.06 & 0.22 & 2 & 7 & 3 & 2 & 190 & 54 & 23.15 & 0.35 & 2 & 26 & 3 & 4 & 150 & 552 & 22.95 & 0.07 & 3 & 3 & 3 & 4 \\
\hline 109 & 56 & 22.98 & 0.03 & 3 & 5 & 4 & 9 & 141 & 58 & 22.60 & 0.09 & 3 & 9 & 3 & 2 & 169 & 59 & 21.34 & 0.06 & 3 & 6 & 5 & 4 \\
\hline 120 & 62 & 22.55 & 0.03 & 3 & 9 & 4 & 5 & 135 & 62 & 21.96 & -0.03 & 3 & 4 & 5 & 4 & 65 & 63 & 23.16 & 0.23 & 2 & 6 & 3 & 4 \\
\hline 93 & 63 & 22.96 & -0.03 & 3 & 8 & 4 & 16 & 141 & 64 & 22.23 & 0.05 & 3 & 2 & 5 & 6 & 22 & 66 & 22.72 & 0.19 & 3 & 2 & 3 & 10 \\
\hline 32 & 67 & 22.37 & 0.02 & 3 & 8 & 3 & 4 & 130 & 67 & 23.25 & 0.04 & 2 & 0 & 3 & 4 & 88 & 68 & 22.98 & 0.47 & 3 & 5 & 3 & 5 \\
\hline 179 & 68 & 22.22 & 0.14 & 3 & 5 & 5 & 5 & 151 & 69 & 19.40 & -0.54 & 3 & 5 & 5 & 4 & 170 & 70 & 22.54 & 0.01 & 3 & 6 & 4 & J \\
\hline 19 & 74 & 21.63 & 0.05 & 3 & 5 & 3 & 2 & 135 & 76 & 21.07 & 0.02 & 3 & 6 & 5 & 4 & 124 & 77 & 22.06 & 0.01 & 2 & 9 & 5 & 5 \\
\hline 143 & 80 & 22.41 & 0.01 & 3 & 5 & 5 & 3 & 171 & 81 & 22.30 & 0.05 & 3 & 11 & 4 & 4 & 31 & 82 & 22.11 & 0.02 & 3 & 8 & 3 & 5 \\
\hline 55 & 84 & 22.89 & 0.07 & 2 & 12 & 3 & 9 & 141 & 88 & 22.35 & 0.08 & 3 & 8 & 5 & 5 & 159 & 88 & 22.84 & 0.09 & 3 & 16 & 4 & 6 \\
\hline 149 & 89 & 23.05 & 0.05 & 3 & 13 & 4 & 6 & 95 & 93 & 22.20 & 0.03 & 2 & 5 & 5 & 5 & 181 & 93 & 22.98 & 0.23 & 3 & 15 & 3 & 9 \\
\hline 119 & 96 & 19.41 & -0.52 & 2 & 5 & 5 & 5 & 82 & 98 & 22.48 & -0.05 & 3 & 0 & 4 & 6 & 140 & 992 & 22.96 & 0.07 & 3 & 10 & 4 & 3 \\
\hline 75 & 104 & 22.53 & 0.69 & 3 & 10 & 4 & 9 & 186 & 104 & 22.29 & 0.04 & 3 & 7 & 5 & 8 & 138 & 1062 & 23.02 & 0.17 & 3 & 8 & 4 & 8 \\
\hline 149 & 110 & 22.73 & -0.12 & 3 & 18 & 4 & 5 & 62 & 111 & 23.04 & 0.43 & 2 & 2 & 3 & 6 & 133 & 112 & 21.34 & 0.04 & 3 & 7 & 5 & 0 \\
\hline 121 & 115 & 21.48 & 0.01 & 3 & 3 & 5 & 3 & 155 & 115 & 22.35 & 0.14 & 3 & 8 & 4 & 4 & 77 & 116 & 22.38 & 0.02 & 3 & 4 & 3 & 4 \\
\hline 92 & 116 & 23.15 & 0.26 & 2 & 25 & 3 & 25 & 57 & 118 & 21.10 & 0.06 & 3 & 5 & 5 & 5 & 115 & 118 & 21.24 & 0.06 & 3 & 6 & 5 & 3 \\
\hline 82 & 119 & 21.44 & 0.05 & 3 & 8 & 5 & 4 & 151 & 119 & 22.41 & 0.00 & 2 & 1 & 5 & 9 & 49 & 1212 & 22.53 & 0.18 & 3 & 20 & 3 & 5 \\
\hline 33 & 123 & 23.16 & 0.02 & 2 & 9 & 3 & 13 & 106 & 125 & 23.02 & 0.06 & 2 & 5 & 4 & 6 & 129 & 126 & 19.91 & 0.25 & 3 & 6 & 5 & 5 \\
\hline 75 & 127 & 22.81 & 0.08 & 3 & 5 & 4 & 10 & 156 & 130 & 22.45 & 0.31 & 3 & 16 & 4 & 4 & 23 & 131 & 23.05 & 0.03 & 3 & 5 & 3 & 5 \\
\hline 68 & 137 & 20.85 & 0.15 & 3 & 6 & 5 & 3 & 115 & 137 & 23.13 & 0.15 & 3 & 9 & 3 & 5 & 196 & 139 & 22.29 & -0.02 & 3 & 4 & 3 & 4 \\
\hline 157 & 140 & 22.52 & 0.05 & 3 & 8 & 4 & 3 & 80 & 141 & 20.71 & 0.18 & 3 & 6 & 5 & 5 & 112 & 143 & 22.61 & 0.05 & 3 & 4 & 4 & 3 \\
\hline 155 & 150 & 20.34 & 0.22 & 3 & 8 & 5 & 5 & 194 & 151 & 21.30 & 0.02 & 3 & 5 & 5 & 3 & 178 & 153 & 22.70 & -0.01 & 3 & 1 & 4 & 1 \\
\hline 60 & 155 & 21.77 & -0.03 & 3 & 1 & 5 & 4 & 51 & 157 & 22.97 & 0.18 & 3 & 2 & 3 & 6 & 128 & 157 & 22.84 & 0.17 & 2 & 8 & 4 & 9 \\
\hline 156 & 157 & 21.97 & 0.03 & 3 & 25 & 5 & 12 & 186 & 158 & 22.15 & -0.01 & 3 & 2 & 5 & 2 & 161 & 159 & 22.02 & 0.12 & 3 & 6 & 3 & 6 \\
\hline 168 & 160 & 20.83 & 0.14 & 3 & 5 & 5 & 3 & 83 & 163 & 21.55 & 0.01 & 3 & 3 & 5 & 3 & 177 & 163 & 22.89 & 0.06 & 3 & 16 & 4 & 6 \\
\hline 55 & 165 & 22.63 & 0.03 & 3 & 7 & 4 & 7 & 19 & 166 & 22.38 & 0.06 & 3 & 13 & 3 & 6 & 153 & 166 & 23.30 & 0.04 & 2 & 7 & 3 & 6 \\
\hline 63 & 172 & 21.98 & 0.06 & 3 & 5 & 5 & 6 & 122 & 172 & 21.46 & 0.01 & 3 & 4 & 5 & 4 & 55 & 174 & 22.05 & 0.00 & 3 & 0 & 5 & 4 \\
\hline 174 & 176 & 22.75 & 0.05 & 3 & 7 & 4 & 4 & 146 & 177 & 22.15 & 0.18 & 3 & 21 & 4 & 7 & 68 & 179 & 21.65 & 0.12 & 3 & 6 & 5 & 4 \\
\hline 99 & 180 & 22.88 & 0.13 & 3 & 4 & 4 & 4 & 85 & 181 & 23.33 & 0.06 & 2 & 9 & 3 & 1 & 161 & 191 & 21.39 & 0.04 & 3 & 8 & 5 & 3 \\
\hline \multicolumn{2}{|r|}{ ield } & & & & & & & 19 & 4 & 21.18 & 0.00 & 2 & 1 & 5 & 11 & 85 & 4 & 22.34 & 0.29 & 2 & 0 & 4 & 14 \\
\hline
\end{tabular}


TABLE IV. (continued)

\begin{tabular}{|c|c|c|c|c|c|c|c|c|c|c|c|c|c|c|c|c|c|c|c|c|c|c|c|}
\hline $\begin{array}{l}\mathrm{x} \\
\text { (pix }\end{array}$ & $x^{y}$ & 1 & $-r$ & \# & $\begin{array}{c}\sigma(r) \\
(\mathrm{mag})\end{array}$ & $\Rightarrow$ & $\begin{array}{c}\sigma(g) \\
(\mathrm{mag})\end{array} \mid$ & $\begin{array}{l}\mathbf{x} \\
\text { (pix }\end{array}$ & & $\mathbf{r}$ & & & (mag) & & (mag) & $\begin{array}{l}\mathbf{x} \\
\text { (pix }\end{array}$ & $\mathbf{y}$ & & $-\mathbf{r}$ & & $\begin{array}{c}\sigma(r) \\
(\mathrm{mag})\end{array}$ & & (D) \\
\hline 32 & 5 & 20.81 & .06 & 3 & 3 & 5 & 6 & 55 & 2 & 23.15 & 0.19 & 2 & 11 & 3 & 11 & 64 & 62 & 22.15 & 0.12 & 2 & 9 & 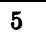 & 11 \\
\hline 18 & 6 & 22.21 & 02 & 3 & 9 & 5 & 5 & (n) & & 22.83 & 0.13 & & 17 & & 7 & 71 & 72 & 89 & 0.05 & 2 & & & 0 \\
\hline 24 & 7 & 22.20 & 03 & 3 & 5 & 4 & 4 & 44 & 82 & & 0.13 & 3 & & & 8 & 2 & 82 & & 0.49 & 2 & 6 & & 2 \\
\hline 37 & 9 & 20.55 & 10 & 3 & 1 & 5 & 6 & 83 & 92 & 22.12 & 0.13 & 3 & 9 & & 10 & 139 & 92 & 21.67 & -0.02 & 3 & 14 & & 6 \\
\hline 31 & 10 & 21.22 & 06 & 3 & 1 & 5 & 6 & 63 & 122 & & 15 & 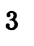 & 9 & & ( & 4 & 12 & 21.82 & 0.14 & 3 & & & 11 \\
\hline 9 & 13 & 22.58 & 16 & 2 & 14 & 4 & 14 & 110 & 132 & 22.60 & 0.14 & 3 & 3 & 4 & 13 & 18 & 13 & 22.69 & 0.04 & 3 & 10 & & \\
\hline 39 & 13 & 22.22 & -0.37 & 2 & 51 & 4 & 6 & 74 & 142 & 22.56 & -0.06 & 2 & 8 & & 35 & 97 & 14 & 22.36 & 0.14 & 3 & & & \\
\hline 40 & 15 & 22.86 & 18 & 3 & 14 & 3 & 9 & 49 & 152 & 22.51 & 0.21 & 3 & 8 & & 7 & 146 & 17 & 21.71 & 0.47 & 3 & & & 64 \\
\hline 32 & 18 & 21.93 & -0.36 & 3 & 59 & 5 & 8 & 79 & 182 & 21.48 & 0.05 & 3 & & & 7 & 13 & 9 & 8 & 0.06 & 3 & & & \\
\hline 17 & 19 & 22.69 & 0.36 & 2 & 6 & 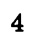 & 12 & 24 & 202 & & 08 & 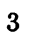 & & & 9 & 48 & 20 & 6 & 02 & 3 & & & 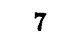 \\
\hline 72 & 20 & 20.30 & 0.06 & 3 & 38 & 5 & 32 & 123 & 212 & 21.77 & 0.16 & 3 & & & 40 & 138 & 22 & 22.65 & 0.06 & 3 & & & 8 \\
\hline 53 & 24 & 22.70 & 04 & 3 & 35 & 3 & $T$ & 130 & 242 & 22.23 & -0.01 & 3 & 1 & & & 62 & 25 & 48 & 00 & 3 & 5 & & 17 \\
\hline 110 & 25 & 19.49 & -0.59 & 3 & 7 & 5 & 7 & 57 & 26 & 22.50 & 0.08 & 2 & 8 & 3 & 7 & 87 & 26 & 22.31 & 0.20 & 3 & & 0 & 13 \\
\hline 80 & 28 & 23.20 & -0.12 & 2 & 3 & 3 & 3 & 23 & 29 & 21.99 & 0.08 & 3 & 3 & & 5 & 116 & 29 & 21.81 & 0.03 & 3 & & & 1 \\
\hline 67 & 30 & 22.36 & 55 & 3 & 5 & 5 & 7 & 123 & 33 & 20.37 & 04 & 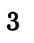 & 6 & & 5 & 55 & 35 & 21.37 & 33 & 0 & & & \\
\hline 62 & 35 & 21.29 & 0.06 & 3 & 7 & 5 & 13 & 95 & 36 & 22.66 & 0.20 & 2 & & & 9 & 134 & 36 & 23.07 & ( & 2 & & & 15 \\
\hline 83 & 38 & 20.91 & -0. & 3 & 6 & 5 & 1. & 49 & 39 & 2 & 2 & 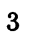 & & & & 105 & 9 & 8 & 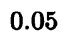 & 3 & & & \\
\hline 97 & 40 & 22.53 & 0.12 & 2 & 2 & 4 & 13 & 130 & 40 & 22.78 & 0.10 & 3 & 18 & 3 & 7 & 17 & 41 & 21.72 & 0.09 & 3 & & & 7 \\
\hline 73 & 42 & 21. & 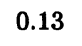 & 3 & 16 & 5 & 14 & 141 & 42 & 5 & 9 & 3 & & & 4 & 31 & 44 & 22.17 & 0.21 & 2 & & & 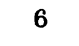 \\
\hline 82 & 44 & 20.13 & 22 & 3 & 1 & 5 & 5 & 69 & 46 & 21.34 & 0.04 & 2 & c & & 4 & 92 & 46 & 22.87 & 0.15 & 3 & 3 & 3 & 41 \\
\hline 104 & 46 & 22.15 & 08 & 3 & 2 & 5 & 6 & 95 & 49 & 22.86 & 0.09 & 2 & 1 & & 1 & 17 & 50 & 3 & 0 & 3 & & & 1 \\
\hline 32 & 50 & 21.80 & 14 & 3 & 5 & 5 & 8 & 39 & 52 & 22.29 & 04 & 3 & 10 & & 12 & 46 & 52 & 0 & ( & 2 & & & 6 \\
\hline 71 & 52 & 22.16 & -0.09 & 3 & 21 & 5 & 8 & 113 & 52 & 19.69 & 0.22 & 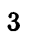 & & & & 10 & 53 & 22.07 & 6 & 2 & & & 10 \\
\hline 56 & 53 & 22.43 & 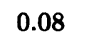 & 3 & 2 & & 9 & 5 & 53 & 22. & 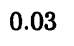 & & & & & 143 & 3 & 04 & 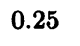 & 3 & & & \\
\hline 21 & 55 & 22.70 & 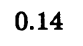 & 3 & 9 & 4 & 10 & 127 & 55 & 21.06 & 3 & 3 & 5 & & 6 & 95 & 56 & 21.21 & 0.06 & 3 & & 5 & 6 \\
\hline 82 & 57 & 22.22 & 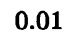 & 3 & 8 & & 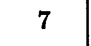 & 52 & 58 & 22.81 & 0 & 3 & & & 1 & 3 & 60 & & c & 3 & & & \\
\hline 41 & 60 & 22.08 & & 3 & 5 & & 6 & 78 & 63 & 22.71 & 00 & 3 & 12 & & 10 & 14 & 64 & 23.35 & 0.13 & 2 & & & 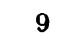 \\
\hline 138 & 65 & 22.49 & 60 & 3 & 23 & 3 & 8 & 53 & 67 & 22.97 & & & & & 22 & 6 & 7 & & & 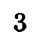 & & & 13 \\
\hline 89 & 67 & 23.30 & 41 & 2 & 3 & 3 & 3 & 124 & 70 & 22.84 & 18 & 2 & 1 & & 3 & 33 & 2 & 21.39 & -0.01 & 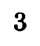 & & & $=$ \\
\hline 44 & 73 & 22.53 & 17 & 3 & 13 & & 4 & 129 & 73 & 22 & & 3 & & & 10 & 35 & 7 & & & - & & & 11 \\
\hline 77 & 80 & 21.81 & -0.04 & 2 & 3 & 5 & 5 & 26 & 82 & 22.90 & 18 & 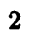 & & & & 50 & 2 & 21.60 & -0 . & 0 & & & \\
\hline 92 & 82 & 21.88 & & 3 & 4 & & 8 & 129 & 82 & 22 & 19 & 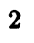 & 8 & & 2 & 135 & 83 & 20.74 & 0.23 & 3 & & & 7 \\
\hline 65 & 86 & 22.75 & 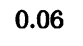 & 3 & 7 & 4 & t & 76 & 94 & 21.62 & & 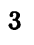 & & & & 137 & 96 & & & 3 & & & 11 \\
\hline 33 & 107 & 22.15 & 11 & 3 & 16 & 5 & 18 & 501 & 108 & 20.84 & 14 & 3 & & & 5 & 37 & 109 & 22.30 & 0.20 & 3 & 10 & & 0 \\
\hline 26 & 110 & 21.70 & 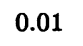 & 3 & 5 & & 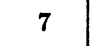 & 741 & 111 & 22 & & 2 & 2 & & 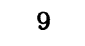 & 119 & 112 & 24 & 0.41 & 3 & & & 11 \\
\hline 113 & 114 & 20.69 & 8 & 3 & 1 & 5 & 6 & 171 & 117 & 22.92 & 0.00 & 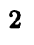 & & & 6 & 36 & 118 & 23.10 & 0.18 & 2 & & & \\
\hline 55 & 122 & 20.43 & & 3 & 2 & 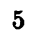 & 5 & 211 & 123 & 22.64 & 0.12 & 3 & 9 & & 13 & 105 & 125 & 22.41 & 0.01 & 3 & & & 8 \\
\hline 57 & 127 & 21.90 & & 2 & 5 & 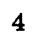 & 6 & 61 & 131 & 22.58 & 0.09 & 2 & 0 & & 10 & 61 & 131 & 20.88 & 0.09 & 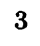 & & & 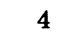 \\
\hline 49 & 133 & 22.79 & 21 & 2 & 1 & 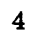 & 10 & 1391 & 137 & 23.07 & 0.02 & 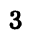 & 9 & & 7 & 14 & 142 & 22.95 & 0.14 & 3 & 10 & 3 & 11 \\
\hline 68 & 142 & 22.86 & 0.06 & 3 & 14 & 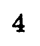 & 8 & 881 & 146 & 23.39 & -0.19 & 2 & $\checkmark$ & & 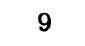 & 23 & 149 & 21.82 & 0.04 & 3 & & & 12 \\
\hline 100 & 9 & 21.17 & 0 & 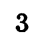 & 2 & 5 & 4 & 131 & 150 & 21.53 & 0.05 & 2 & 8 & & 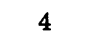 & 30 & 152 & 20.74 & 0.15 & 3 & & & 3 \\
\hline 116 & 154 & 22.39 & 0.22 & 3 & 2 & 0 & 2 & 70 & 159 & 21.90 & 0.03 & ( & 4 & ( & 6 & 110 & 159 & 21.74 & 0.00 & 3 & & & 5 \\
\hline 28 & 160 & 22.21 & -0.05 & 3 & 13 & 5 & 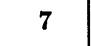 & 43 & 161 & 22.09 & 0.07 & . & & & 0 & 28 & 166 & 21.90 & 0.05 & 3 & 9 & & 6 \\
\hline 131 & 167 & 21.79 & -0.22 & 3 & 37 & - & 7 & 331 & 171 & 23.08 & -0.07 & 3 & 10 & & 5 & 137 & 171 & 22.51 & 0.23 & 5 & 4 & & 12 \\
\hline 75 & 175 & 23. & & 2 & 20 & 3 & 7 & 1 & 179 & 22.37 & 0.12 & 2 & 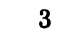 & & - & 80 & 182 & 23.35 & 0.07 & 2 & 8 & & 13 \\
\hline 125 & 186 & 21.33 & 0.00 & 3 & 2 & 0 & 4 & 80 & 189 & 22.29 & -0.01 & 3 & 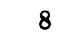 & & 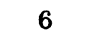 & 90 & 193 & 21.97 & -0.06 & 3 & 0 & & 4 \\
\hline & Field & & & & & & & 29 & & & & 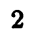 & 12 & & 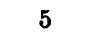 & 46 & 14 & 22.74 & 0.64 & 3 & 19 & & 10 \\
\hline 160 & 37 & 22.95 & 0.00 & 3 & 21 & & 10 & 185 & 37 & 21.79 & 0.65 & 3 & 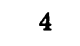 & 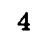 & 12 & 39 & 48 & 22.89 & 0.01 & 2 & 6 & & 6 \\
\hline 34 & 55 & 22.41 & 0.17 & - & 16 & 4 & 7 & 13 & 59 & 21.75 & 0.09 & - & & & 7 & 147 & 61 & 21.96 & -0.05 & 3 & 11 & & 8 \\
\hline
\end{tabular}


TABLE IV. (continued)

\begin{tabular}{|c|c|c|c|c|c|c|c|c|c|c|c|c|c|c|c|c|c|c|c|c|c|c|c|}
\hline $\begin{array}{l}\mathrm{x} \\
(\mathrm{pi}\end{array}$ & ix) & $r$ & g-r & \# & $\begin{array}{c}\sigma(r) \\
(\mathrm{mag})\end{array}$ & \# & $\begin{array}{c}\sigma(g) \\
(\mathrm{mag})\end{array}$ & & $i x)^{y}$ & $r$ & g-r & \# & $\begin{array}{c}\sigma(r) \\
(\mathrm{mag})\end{array}$ & \# & $\begin{array}{c}\sigma(g) \\
(\mathrm{mag})\end{array}$ & & ${ }_{x)}^{y}$ & $\mathrm{r}$ & $\mathrm{g}-\mathrm{r}$ & $\#$ & $\begin{array}{c}\sigma(r) \\
(\mathrm{mag})\end{array}$ & \# & $\begin{array}{c}\sigma(g) \\
(\mathrm{mag})\end{array}$ \\
\hline 74 & 62 & 21.82 & 0.43 & 3 & 7 & 5 & 11 & 158 & 68 & 22.43 & -0.02 & 3 & 9 & 5 & 9 & 23 & 69 & 22.57 & 0.43 & 3 & 24 & 4 & 13 \\
\hline 117 & 77 & 21.53 & -0.03 & 3 & 7 & 5 & 7 & 69 & 80 & 21.66 & 0.00 & 3 & 3 & 5 & 6 & 138 & 81 & 22.79 & 0.10 & 3 & 7 & 4 & 10 \\
\hline 50 & 82 & 23.05 & 0.10 & 3 & 8 & 4 & 11 & 101 & 82 & 22.08 & 0.04 & 3 & 4 & 5 & 7 & 145 & 99 & 22.93 & 0.08 & 3 & 13 & 4 & 11 \\
\hline 118 & 105 & 21.91 & 0.06 & 3 & 4 & 5 & 12 & 94 & 106 & 23.19 & 0.03 & 3 & 2 & 3 & 18 & 66 & 112 & 22.53 & 0.24 & 3 & 9 & 4 & 17 \\
\hline 47 & 113 & 23.13 & 0.25 & 2 & 4 & 3 & 18 & 78 & 115 & 22.17 & -0.36 & 3 & 19 & 5 & 88 & 58 & 117 & 22.78 & 0.10 & 3 & 15 & 4 & 9 \\
\hline 158 & 118 & 21.81 & 0.03 & 3 & 6 & 5 & 8 & 83 & 119 & 20.57 & 0.20 & 3 & 3 & 3 & 4 & 23 & 121 & 22.44 & 0.06 & 3 & 3 & 4 & 6 \\
\hline 9 & 122 & 22.79 & 0.14 & 3 & 12 & 4 & 9 & 123 & 126 & 23.08 & 0.24 & 3 & 20 & 3 & 11 & 90 & 131 & 21.95 & 0.08 & 3 & 4 & 4 & 8 \\
\hline 104 & 134 & 20.12 & 0.28 & 3 & 4 & 5 & 7 & 51 & 138 & 22.90 & 0.02 & 3 & 12 & 4 & 6 & 141 & 138 & 22.00 & 0.03 & 3 & 6 & 5 & 9 \\
\hline 155 & 143 & 22.86 & 0.27 & 3 & 8 & 3 & 9 & 57 & 144 & 23.10 & -0.07 & 3 & 44 & 4 & 9 & 9 & 145 & 23.11 & 0.24 & 2 & 0 & 3 & 11 \\
\hline 50 & 146 & 22.09 & 0.15 & 3 & 2 & 5 & 13 & 175 & 147 & 23.22 & 0.19 & 3 & 17 & 3 & 15 & 157 & 150 & 23.00 & 0.13 & 3 & 9 & 4 & 20 \\
\hline 103 & 152 & 22.27 & 0.22 & 2 & 8 & 3 & 6 & 81 & 156 & 21.81 & 0.09 & 3 & 5 & 5 & 8 & 189 & 157 & 20.25 & 0.27 & 2 & 4 & 4 & 6 \\
\hline 22 & 158 & 21.40 & 0.10 & 3 & 3 & 5 & 7 & 118 & 158 & 21.46 & 0.12 & 3 & 3 & 5 & 9 & 77 & 163 & 22.66 & 0.06 & 3 & 7 & 3 & 7 \\
\hline 126 & 163 & 22.68 & 0.08 & 3 & 12 & 4 & 14 & 183 & 163 & 22.74 & 0.24 & 3 & 3 & 3 & 8 & 67 & 164 & 22.40 & 0.14 & 3 & 7 & 4 & 11 \\
\hline 144 & 166 & 21.91 & 0.05 & 3 & 5 & 5 & 6 & 61 & 170 & 21.20 & 0.14 & 3 & 2 & 5 & 6 & 54 & 173 & 22.42 & 0.13 & 3 & 6 & 4 & 8 \\
\hline 121 & 174 & 22.55 & 0.00 & 3 & 5 & 4 & 9 & 153 & 175 & 23.23 & -0.19 & 3 & 39 & 4 & 8 & 108 & 176 & 22.44 & 0.42 & 3 & 2 & 4 & 23 \\
\hline 140 & 178 & 22.66 & 0.19 & 3 & 8 & 4 & 8 & 41 & 180 & 21.57 & 0.09 & 2 & 0 & 5 & 9 & 15 & 181 & 23.11 & 0.14 & 2 & 4 & 3 & 12 \\
\hline 49 & 181 & 22.32 & 0.10 & 3 & 8 & 4 & 4 & 105 & 182 & 22.10 & 0.82 & 3 & 6 & 4 & 5 & 66 & 184 & 22.36 & 0.14 & 3 & 4 & 4 & 8 \\
\hline 44 & 187 & 22.85 & 0.22 & 3 & 13 & 4 & 5 & 51 & 187 & 22.73 & 0.12 & 3 & 10 & 4 & 8 & 88 & 188 & 21.69 & 0.12 & 3 & 2 & 5 & 10 \\
\hline 17 & 191 & 21.83 & 0.18 & 2 & 1 & 5 & 6 & 68 & 191 & 22.62 & -0.97 & 3 & 136 & 5 & 6 & 167 & 194 & 22.59 & 0.07 & 3 & 11 & 4 & 7 \\
\hline 162 & 196 & 22.34 & 0.06 & 2 & 2 & 4 & 11 & & & & & & & & & & & & & & & & \\
\hline
\end{tabular}

outskirts of NGC 7006 are shown in Fig. 8. The solid line is the mean subgiant locus from Fig. 7. The main-sequence turnoff has been detected in all three clusters.

Figure 9 shows the NGC 5466 data transformed to the $B, V$ system using the equations given in Sec. II. The thick, solid line is the mean locus of the faintest stars in the photographic survey by Buonanno et al. (1984). They obtained a color-magnitude diagram of NGC 5466 that reaches to $B=19$ mag but relies on transformations of $P, V$ photoelectric photometry of nine stars in the field of this cluster by Cuffey (1961) to define the magnitude system. The giant branches in both studies merge smoothly together in color. Buonanno et al. (1984) review the metallicity and reddening determinations for NGC 5466. $E(B-V)$ is known to be less than $0.03 \mathrm{mag}$, while published $[\mathrm{Fe} / \mathrm{H}]$ values range from -2.2 to $-1.5 \mathrm{dex}$; the median of the ten published determinations, not all of which are independent, is -1.9 .

We adopt for all the clusters the distance moduli $(m-M)_{V}$ tabulated by Harris and Racine (1979), which assume $M_{V}$ (RR Lyraes) $=0.6 \mathrm{mag}$ as well as their tabulated reddening value $E(B-V)=0.05 \mathrm{mag}$ for NGC 5466 . We then superpose onto the NGC $5466 \mathrm{c}-\mathrm{m}$ diagram the $16 \times 10^{9} \mathrm{yr}$ isochrone for $Z=10^{-4}$ and helium content $Y=0.20$ from VandenBerg and Bell (1985). The isochrones near the main sequence are somewhat bluer (by about 0.08 $\mathrm{mag}$ ) than the locus of stars in very metal-poor clusters (including M92) with adequate photometry. However, the turnoff luminosity itself of NGC 5466 requires an age of at least $16 \times 10^{9} \mathrm{yr}$, comparable to that of the globular clusters in the inner halo. There is no sign that NGC 5466 is younger than the average globular cluster.

It is possible that the color discrepancy between the isochrones and the clusters $B-V$ colors near the turnoff result from a problem in the theoretical isochrones, as several of the metal-poor clusters in VandenBerg's (1983) Fig. 5 (as well as all three clusters studied in this paper) show the same phenomenon. However, the use of accurate model atmospheres in VandenBerg and Bell (1985) should have eliminated any significant error in color. It is also possible that the photometric errors are larger than we believe, although this too seems unlikely. A more reasonable explanation for at least part of the color discrepancy lies in our neglect of the metallicity dependence in the transformation from $g-r$ to $B-V$ color. The effective wavelengths of $g$ and $r$ (4930 and $6650 \AA$, respectively) are both redder than that of $B$ at about $4400 \AA$. Although the standards defining the Thuan-Gunn system are white dwarfs and metal-poor subdwarfs, their mean metallicity is higher than that of the metal-poor globular clusters discussed in this paper; neglecting the reduced line blanketing throughout the $B$ filter bandpass in the globular cluster stars will lead to $B-V$ colors for them that are too red. The fact that the $B-V$ colors of the M3 stars predicted from our measured $g-r$ colors were in the mean 0.085 mag redder than those measured by Sandage (1970) (see Sec. II $b$ ) supports this hypothesis. One should also note that the transformation given by Kent (1985) between $g-r$ and $B-V$ colors derived from photometry of stars with near solar metallicity has even redder $B-V$ colors for a given $g-r$ color than do the equations used in this paper and derived directly from the bright standards of the ThuanGunn system, which are somewhat metal poor in the mean.

Ideally, $B-V$ must be determined from a color that more closely spans the $B$ and $V$ wavelength bandpasses (i.e., $v-g$ in the Thaun-Gunn system), rather than be extrapolated from a continuum slope defined at longer wavelengths only. It would also be desirable for the VandenBerg and Bell (1985) isochrones to be converted into the Thuan-Gunn system, since this photometric system is finding widespread use with CCD detectors.

The $B, V$ c-m diagram of NGC 6229 is shown in Fig. 10. 
TABLE V. Magnitudes of NGC 7006 stars.

\begin{tabular}{|c|c|c|c|c|c|c|c|c|c|c|c|c|c|c|c|c|c|c|c|c|c|c|c|}
\hline & $\left.{ }_{i x}\right)^{y}$ & $\mathbf{r}$ & g-r & $\#$ & $\begin{array}{c}\sigma(r) \\
(\mathrm{mag})\end{array}$ & $\#$ & $\begin{array}{c}\sigma(g) \\
(\mathrm{mag})\end{array}$ & & ix) & $\mathrm{r}$ & g-r & \# & $\begin{array}{c}\sigma(r) \\
(\mathrm{mag})\end{array}$ & $\#$ & $\begin{array}{c}\sigma(g) \\
(\mathrm{mag})\end{array}$ & $\begin{array}{l}\mathbf{x} \\
\text { (pis }\end{array}$ & $\mathbf{x}^{\mathbf{y}}$ & $\mathbf{r}$ & g-r & \# & $\begin{array}{c}\sigma(r) \\
(\mathrm{mag})\end{array}$ & $\#$ & $\begin{array}{c}\sigma(g) \\
\text { (mag) }\end{array}$ \\
\hline & Field & & & & & & & 77 & 4 & 22.51 & 0.23 & 1 & 0 & 3 & 8 & 98 & 5 & 21.70 & 0.31 & 2 & 2 & 4 & 4 \\
\hline 42 & 7 & 23.28 & 0.39 & 2 & 19 & 3 & 9 & 144 & 7 & 22.02 & 0.09 & 2 & 5 & 4 & 2 & 154 & 7 & 22.29 & -0.08 & 1 & 0 & 4 & 17 \\
\hline 10 & & 23.14 & 0.36 & 1 & 0 & 2 & 1 & 133 & 8 & 20.73 & 0.27 & 2 & 1 & 4 & 2 & 150 & 8 & 23.05 & -0.82 & 2 & 20 & 2 & 5 \\
\hline 21 & & 23.45 & 0.42 & 2 & 11 & 3 & 28 & 28 & 11 & 22.71 & 0.13 & 2 & 18 & 3 & 5 & 50 & 11 & 22.63 & 0.17 & 2 & 2 & 3 & 15 \\
\hline 154 & 11 & 23.07 & -0.31 & 2 & 5 & 2 & 35 & 34 & 13 & 22.90 & 0.30 & 2 & 11 & 2 & 1 & 110 & 13 & 21.93 & 0.12 & 2 & 8 & 4 & 2 \\
\hline 19 & 17 & 22.49 & 0.16 & 2 & 8 & 4 & 6 & 66 & 17 & 22.76 & 0.21 & 2 & 14 & 2 & 11 & 82 & 18 & 22.30 & 0.16 & 2 & 6 & 4 & 1 \\
\hline 12 & 19 & 23.44 & 0.20 & 1 & 0 & 3 & 26 & 31 & 21 & 21.84 & -0.06 & 2 & 1 & 4 & 3 & 8 & 27 & 22.86 & 0.29 & 2 & 0 & 3 & 11 \\
\hline 149 & 29 & 23.36 & 0.26 & 2 & 10 & 3 & 12 & 106 & 30 & 23.13 & 0.38 & 1 & 0 & 3 & 14 & 122 & 30 & 23.38 & 0.46 & 1 & 0 & 2 & 17 \\
\hline 52 & 32 & 22.72 & 0.22 & 2 & 3 & 4 & 11 & 112 & 32 & 23.35 & 0.29 & 1 & 0 & 2 & 4 & 131 & 32 & 22.72 & 0.19 & 2 & 8 & 3 & 6 \\
\hline 37 & & 23.08 & 0.21 & 2 & 10 & 4 & 30 & 144 & 37 & 22.56 & 0.23 & 2 & 1 & 4 & 4 & 71 & 39 & 23.06 & -0.01 & 2 & 25 & 3 & 9 \\
\hline 45 & & 21.90 & 0.19 & 2 & 4 & 4 & 5 & 68 & 47 & 23.25 & 0.24 & 2 & 19 & 2 & 3 & 47 & 48 & 22.93 & 0.38 & 2 & 2 & 3 & 8 \\
\hline 38 & & 22.77 & 0.21 & 2 & 1 & 4 & 8 & 99 & 49 & 23.31 & -0.32 & 2 & 50 & 3 & 6 & 82 & 50 & 21.81 & 0.21 & 2 & 0 & 4 & 4 \\
\hline 130 & & 19.81 & 0.31 & 2 & 4 & 4 & 0 & 62 & 57 & 23.37 & 0.34 & 1 & 0 & 3 & 7 & 45 & 61 & 23.36 & 0.35 & 2 & 16 & 2 & 23 \\
\hline 99 & & 23.30 & 0.61 & 1 & 0 & 3 & 40 & 140 & 61 & 23.31 & 0.39 & 2 & 4 & 3 & 14 & 135 & 65 & 22.80 & 1.15 & 2 & 1 & 2 & 6 \\
\hline 149 & & 23.24 & 0.29 & 1 & 0 & 2 & 7 & 71 & 67 & 19.83 & 1.13 & 2 & 2 & 4 & 3 & 85 & 67 & 22.64 & 0.21 & 2 & 0 & 4 & 10 \\
\hline 36 & 79 & 23.48 & 0.49 & 2 & 16 & 2 & 41 & 56 & 79 & 18.95 & 0.09 & 2 & 3 & 4 & 1 & 94 & 84 & 22.82 & 1.37 & 2 & 5 & 2 & 0 \\
\hline 127 & & 22.32 & 0.21 & 2 & 1 & 4 & 9 & 72 & 86 & 22.98 & 0.10 & 2 & 18 & 3 & 6 & 41 & 87 & 23.14 & 0.39 & 2 & 10 & 3 & 12 \\
\hline 35 & & 23.49 & 0.52 & 1 & 0 & 2 & 21 & 140 & 89 & 23.19 & 0.34 & 1 & 0 & 3 & 13 & 39 & 91 & 23.47 & 0.26 & 1 & 0 & 2 & 34 \\
\hline 107 & 97 & 23.12 & 0.31 & 2 & 21 & 3 & 8 & 31 & 98 & 22.27 & 0.09 & 2 & 8 & 4 & 9 & 101 & 100 & 22.92 & 0.20 & 2 & 10 & 4 & 17 \\
\hline 13 & 101 & 21.19 & 0.50 & 2 & 0 & 4 & 25 & 9 & 106 & 22.07 & 0.08 & 2 & 6 & 3 & 10 & 22 & 108 & 23.45 & 0.23 & 1 & 0 & 3 & 10 \\
\hline 72 & 108 & 22.88 & 0.20 & 2 & 11 & 3 & 15 & 40 & 119 & 23.24 & 0.67 & 2 & 5 & 2 & 15 & 116 & 119 & 22.89 & 0.85 & 2 & 10 & 3 & 14 \\
\hline 7 & 126 & 21.75 & 0.13 & 2 & 1 & 4 & 2 & 109 & 132 & 22.38 & 0.20 & 2 & 8 & 4 & 20 & 26 & 135 & 22.68 & 0.06 & 2 & 2 & 4 & 10 \\
\hline 72 & 140 & 23.07 & 0.01 & 2 & 2 & 3 & 4 & 64 & 146 & 19.61 & 0.31 & 2 & 2 & 4 & 2 & 115 & 151 & 21.71 & 0.83 & 2 & 4 & 4 & 11 \\
\hline 9 & 156 & 22.21 & 0.13 & 2 & 2 & 4 & 12 & 86 & 156 & 21.64 & 1.17 & 2 & 1 & 4 & 4 & 64 & 158 & 22.52 & 0.13 & 2 & 5 & 4 & 2 \\
\hline 29 & 159 & 23.19 & 0.54 & 1 & 0 & 3 & 26 & 19 & 167 & 18.80 & 0.15 & 2 & 2 & 4 & 22 & 37 & 182 & 21.73 & 0.16 & 2 & 4 & 4 & 2 \\
\hline 77 & 183 & 22.23 & 0.07 & 2 & 7 & 4 & 7 & 70 & 184 & 23.21 & -0.09 & 2 & 2 & 4 & 33 & & & & & & & & \\
\hline & Field & & & & & & & 77 & & 21.54 & 0.23 & 2 & 5 & 3 & 3 & 82 & 17 & 19.87 & 0.19 & 2 & 3 & 3 & 1 \\
\hline 115 & & 23.07 & 0.27 & 1 & 0 & 3 & 10 & 37 & 45 & 23.45 & 0.40 & 1 & 0 & 2 & 17 & 67 & 47 & 23.28 & 0.21 & 1 & 0 & 3 & 9 \\
\hline 104 & & 22.72 & 1.66 & 2 & 8 & 2 & 20 & 57 & 66 & 19.45 & 1.41 & 2 & 4 & 3 & 2 & 89 & 66 & 22.53 & 1.85 & 2 & 4 & 2 & 14 \\
\hline 12 & & 22.97 & 0.12 & 1 & 0 & 3 & 2 & 126 & 68 & 22.83 & 0.44 & 2 & 9 & 3 & 4 & 21 & 69 & 20.29 & 0.63 & 2 & 5 & 3 & 2 \\
\hline 6 & 70 & 21.18 & 1.51 & 2 & 7 & 3 & 9 & 116 & 78 & 23.38 & 1.43 & 1 & 0 & 2 & 4 & 38 & 95 & 22.43 & 1.55 & 2 & 4 & 2 & 14 \\
\hline 13 & 126 & 22.34 & 1.32 & 2 & 5 & 3 & 15 & 66 & 139 & 20.63 & 1.11 & 2 & 4 & 3 & 2 & 86 & 142 & 23.48 & 0.50 & 1 & 0 & 3 & 13 \\
\hline 19 & 146 & 20.89 & 0.32 & 2 & 3 & 3 & 3 & 41 & 159 & 19.09 & 0.88 & 2 & 5 & 3 & 3 & 63 & 161 & 21.62 & 0.23 & 2 & 1 & 3 & 5 \\
\hline 79 & 163 & 22.94 & 0.24 & 2 & 9 & 3 & 1 & 53 & 176 & 20.79 & 0.85 & 2 & 4 & 3 & 3 & 9 & 193 & 21.56 & 0.20 & 2 & 2 & 3 & 0 \\
\hline & Field & & & & & & & 98 & 13 & 21.07 & 1.11 & 2 & 3 & 3 & 1 & 114 & 16 & 21.19 & 0.37 & 2 & 3 & 3 & 1 \\
\hline 139 & 17 & 22.65 & 0.07 & 1 & 0 & 3 & 5 & 25 & 22 & 19.46 & 1.33 & 2 & 0 & 3 & 0 & 89 & 28 & 21.43 & 0.39 & 2 & 0 & 2 & 0 \\
\hline 77 & 30 & 21.47 & 0.43 & 2 & 0 & 3 & 2 & 116 & 34 & 22.95 & 0.42 & 1 & 0 & 3 & 9 & 100 & 48 & 20.45 & 0.77 & 2 & 0 & 3 & 1 \\
\hline 23 & 52 & 22.61 & 0.27 & 2 & 1 & 3 & 7 & 14 & 68 & 22.58 & 0.30 & 1 & 0 & 3 & 7 & 64 & 70 & 23.47 & 0.34 & 1 & 0 & 3 & 17 \\
\hline 109 & 72 & 23.19 & 0.39 & 1 & 0 & 3 & 7 & 145 & 73 & 23.02 & 0.31 & 1 & 0 & 3 & 4 & 64 & 82 & 20.13 & 1.44 & 2 & 1 & 3 & 1 \\
\hline 147 & 87 & 23.30 & 0.16 & 1 & 0 & 3 & 6 & 11 & 92 & 22.35 & 0.20 & 2 & 2 & 3 & 1 & 143 & 97 & 23.01 & 0.20 & 1 & 0 & 3 & 6 \\
\hline 34 & 102 & 22.90 & 1.10 & 1 & 0 & 2 & 38 & 96 & 106 & 23.25 & 0.24 & 1 & 0 & 2 & 4 & 901 & 108 & 21.01 & 0.37 & 2 & 0 & 3 & 2 \\
\hline 23 & 120 & 19.96 & 0.69 & 2 & 0 & 3 & 0 & 97 & 134 & 19.21 & -0.26 & 2 & 1 & 3 & 1 & 126 & 138 & 22.60 & 1.42 & 1 & 0 & 3 & 21 \\
\hline 148 & 138 & 23.24 & 0.17 & 1 & 0 & 3 & 6 & 116 & 146 & 23.32 & 0.21 & 1 & 0 & 3 & 14 & 135 & 148 & 20.78 & 0.68 & 2 & 1 & 3 & 5 \\
\hline 29 & 171 & 20.80 & 0.86 & 2 & 0 & 3 & 1 & 101 & 172 & 22.99 & 0.06 & 1 & 0 & 3 & 12 & 145 & 175 & 21.08 & 0.28 & 2 & 2 & 3 & 2 \\
\hline 123 & 182 & 23.35 & 0.52 & 1 & 0 & 2 & 18 & 55 & 201 & 21.91 & 1.49 & 2 & 1 & 3 & 5 & 802 & 215 & 21.10 & 1.26 & 2 & 1 & 3 & 6 \\
\hline 50 & 222 & 22.44 & 1.37 & 2 & 0 & 3 & 12 & 24 & 227 & 22.51 & 1.25 & 2 & 2 & 3 & 13 & & & & & & & & \\
\hline
\end{tabular}




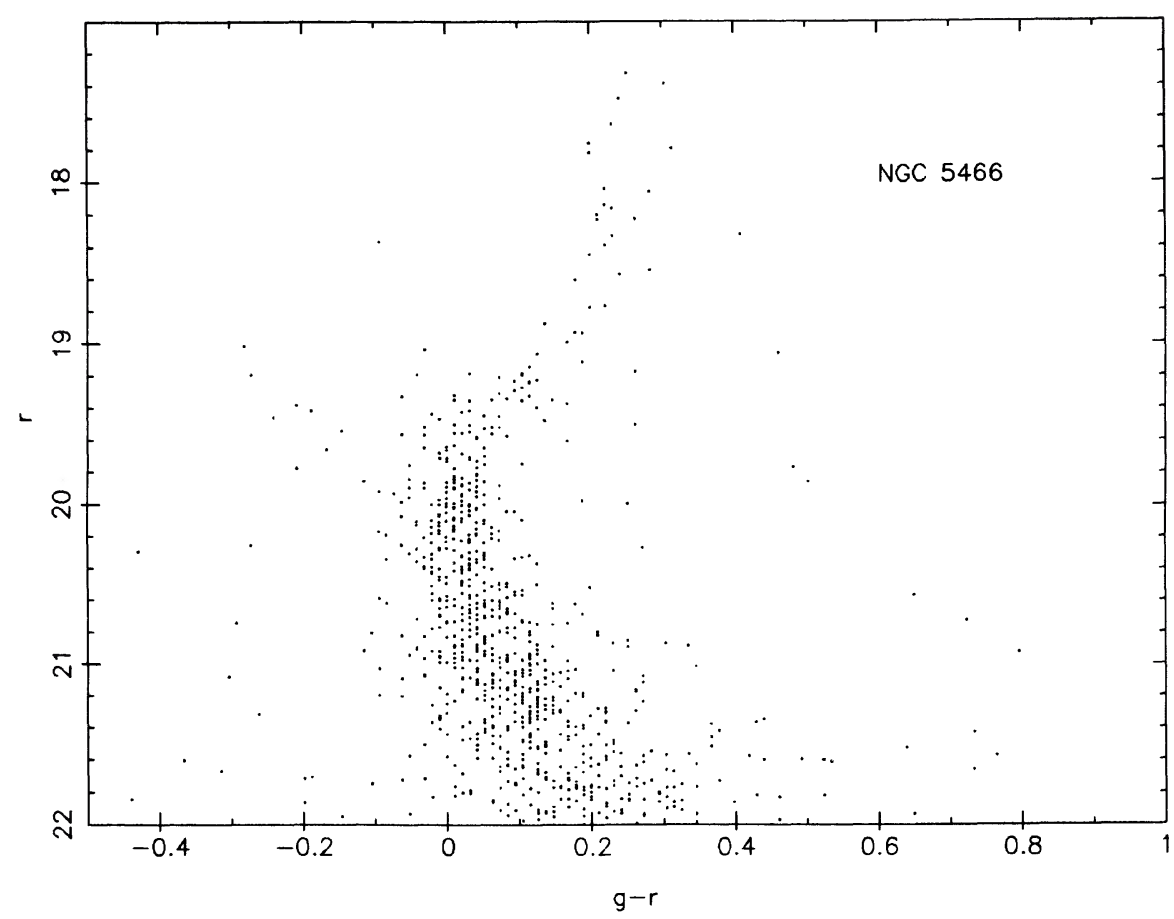

FIG. 4. The $g, r$ color-magnitude diagram for stars in NGC 5466 measured on at least two of the $g$ frames is plotted.

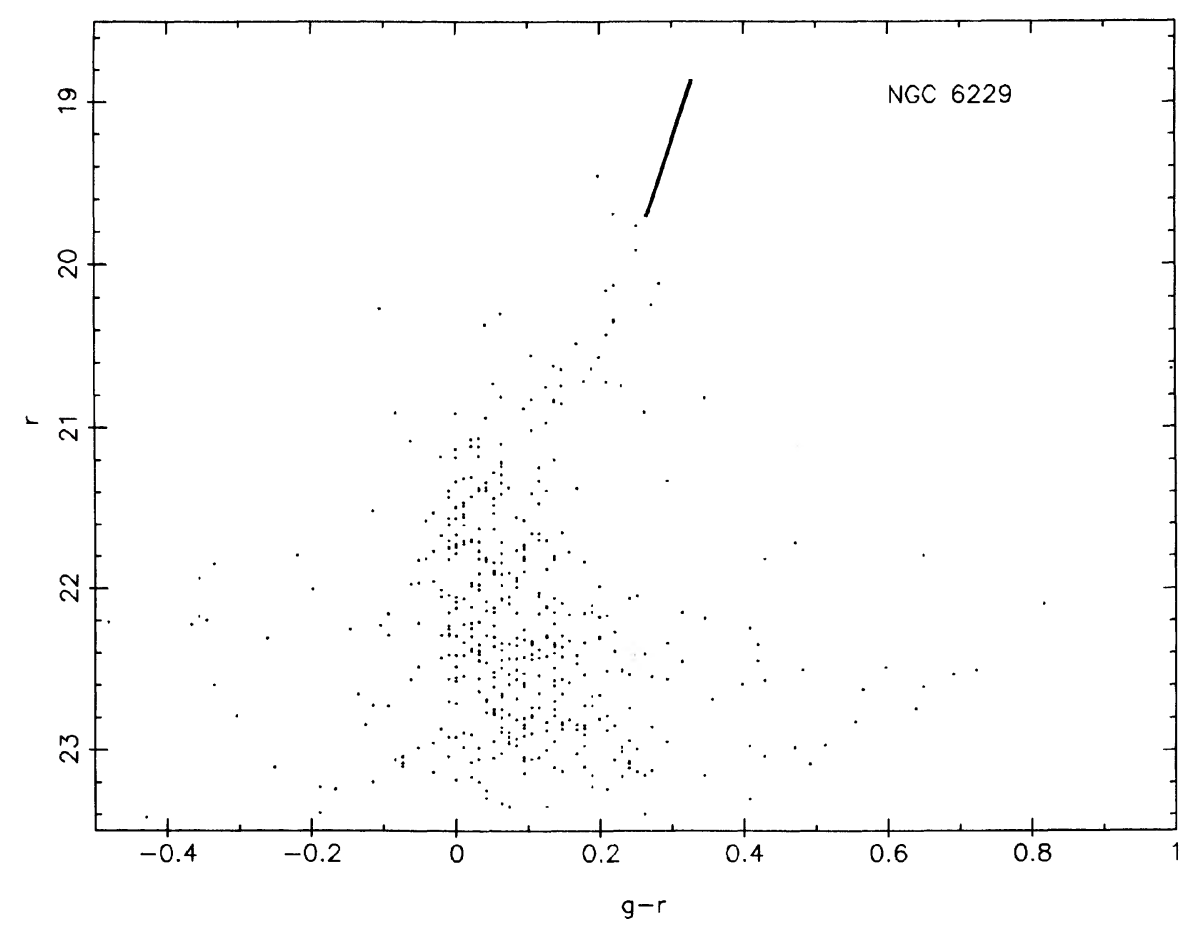

FIG. 5. The $g, r$ color-magnitude diagram for stars in NGC 6229 detected on at least three of the $g$ and two of the $r$ frames is shown. The solid line is the mean locus of bright giants from Fig. 6. 


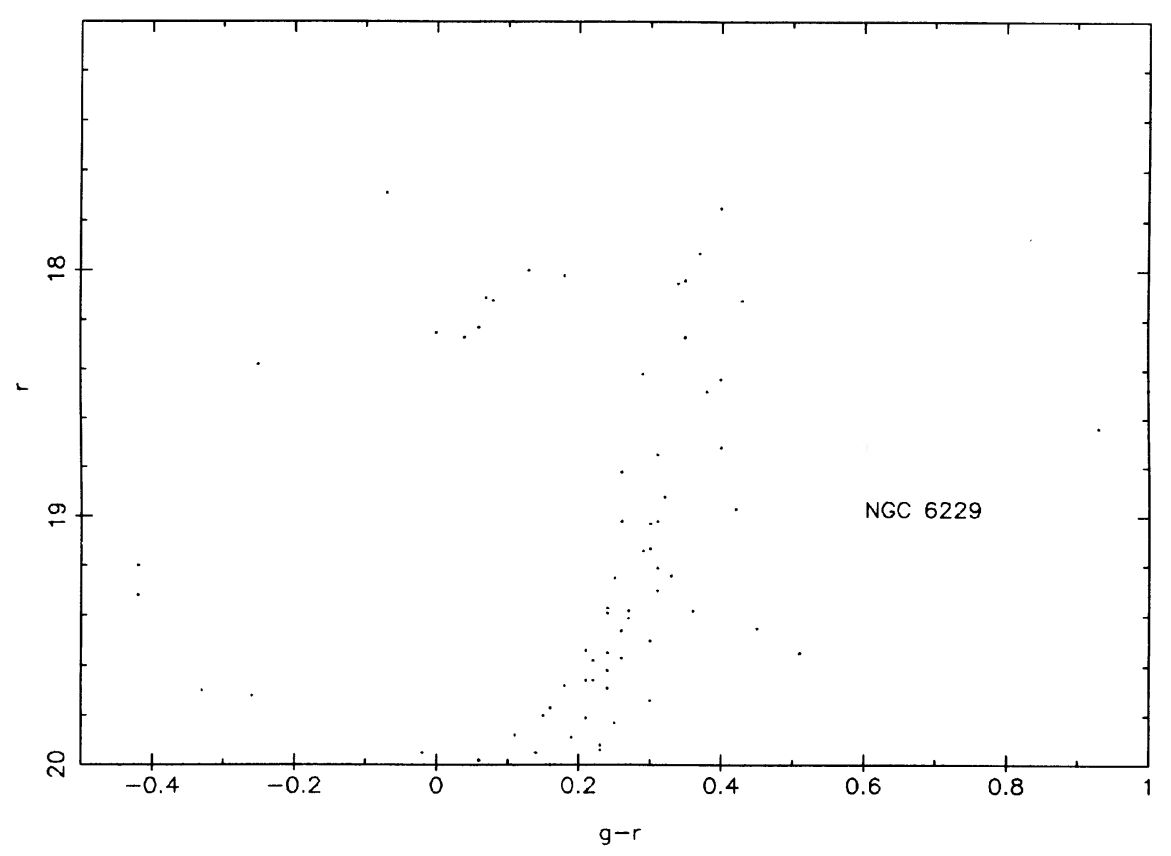

Fig. 6. $g, r \mathrm{c}-\mathrm{m}$ diagram for stars in a field close to the center of NGC 6229 .

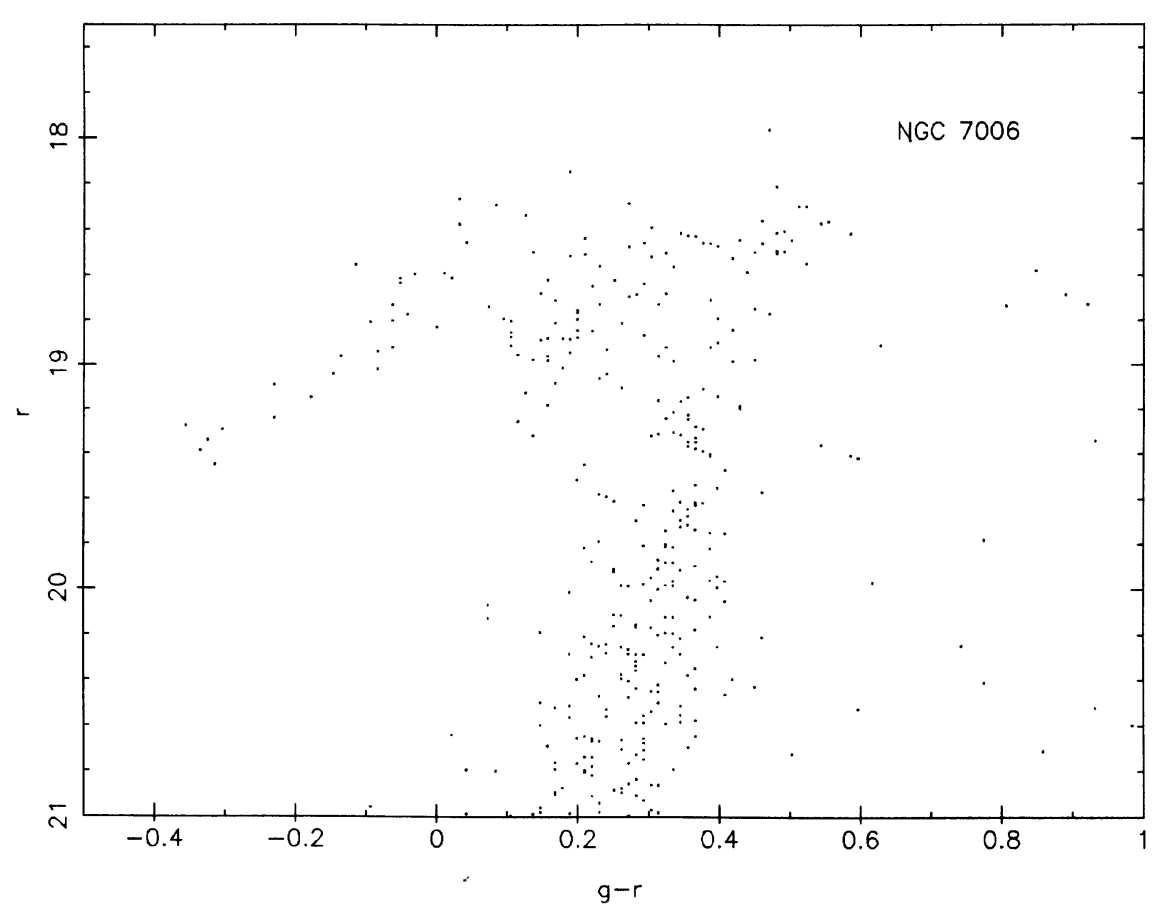

FIG. 7. $g, r$ color-magnitude diagram for a region close to the center of NGC 7006. 


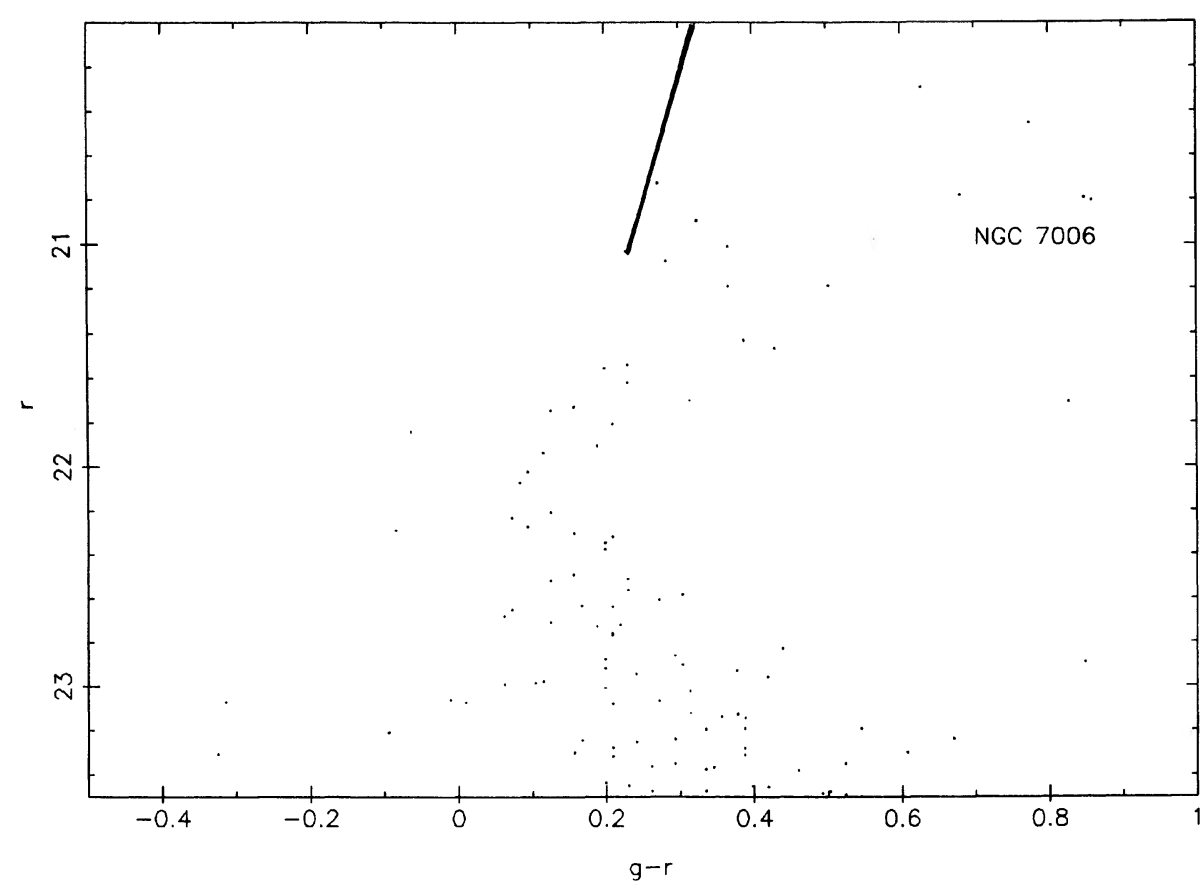

Fig. 8. $g, r \mathrm{c}-\mathrm{m}$ diagram for stars in the three outlying fields of NGC 7006 measured on at least two of the $g$ frames.

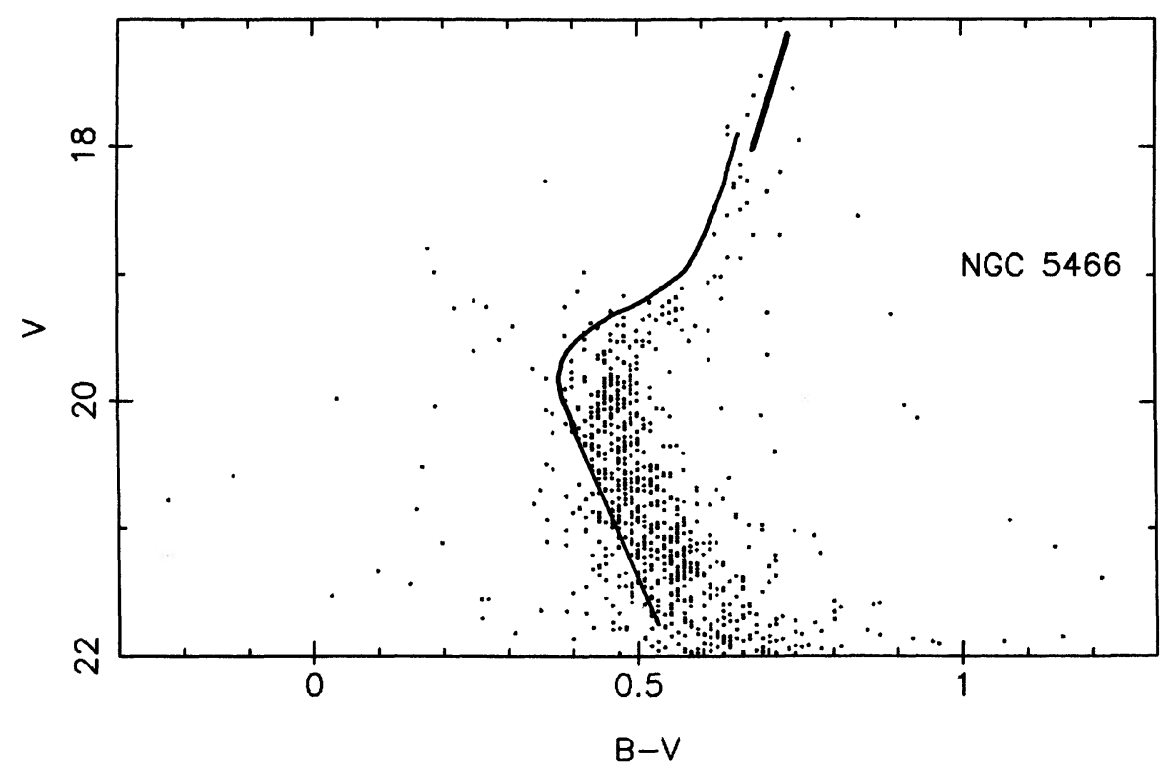

FIG. 9. $B, V$ color-magnitude diagram for NGC 5466. The thick, solid line is the mean locus of the bright giants from Buonanno et al. (1984). The thin solid line is the isochrone of VandenBerg and Bell (1985) for $Z=10^{-4}, Y=0.20$, and age $=16 \times 10^{9} \mathrm{yr}$ 


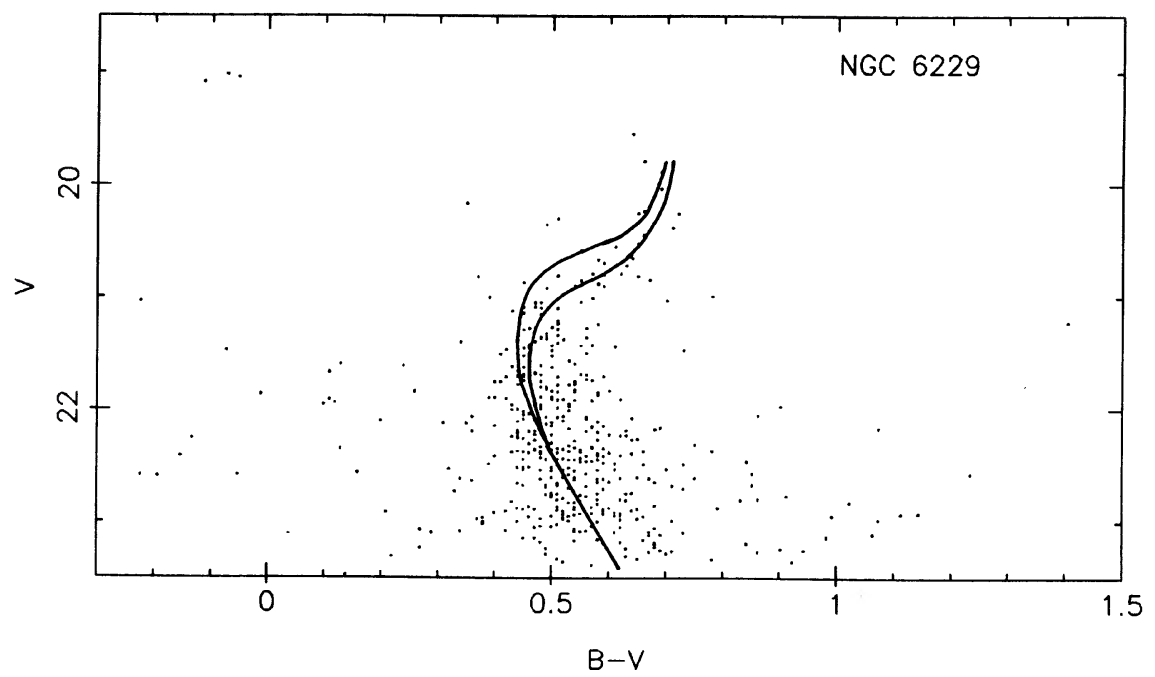

FIG. 10. $B, V$ color-magnitude diagram for NGC 6229. The thick, solid line is the mean locus of the brighter giants from our frames. The thinner lines represent isochrones with ages of 14 and 18 billion years with $Z=3 \times 10^{-4}$ and $Y=0.20$ from VandenBerg and Bell (1984) offset 0.10 mag redder in $B-V$.
Metallicity determinations for NGC 6229 are reviewed by Pilachowski (1983); they range from $[\mathrm{Fe} / \mathrm{H}]=-1.3$ to - 1.5 dex. Zinn (1980) found $E(B-V)=0.01 \mathrm{mag}$, and a very low reddening is supported by the relevant entries near NGC 6229 from Burstein and Heiles' (1983) compilation of H I column densities along the line of sight to galaxies. Superposed on Fig. 8 are isochrones from VandenBerg and Bell (1984) (basically differing only in detail from those of VandenBerg 1983) for a helium content $Y=0.20$ and $Z=3 \times 10^{-4}$ and for ages of 14 and 18 billion years. These isochrones have been offset to redder $B-V$ colors by 0.10 mag to produce a reasonable fit. The deduced age is $17 \pm 3$ billion years, comparable to those of the nearer galactic globulars discussed by VandenBerg (1983).

The $B-V \mathrm{c}-\mathrm{m}$ diagram for the three outlying fields in NGC 7006 is shown in Fig. 11. The thick, solid line is the mean location of the subgiants from the sample in the inner part of the cluster. The metallicity of NGC 7006 has been

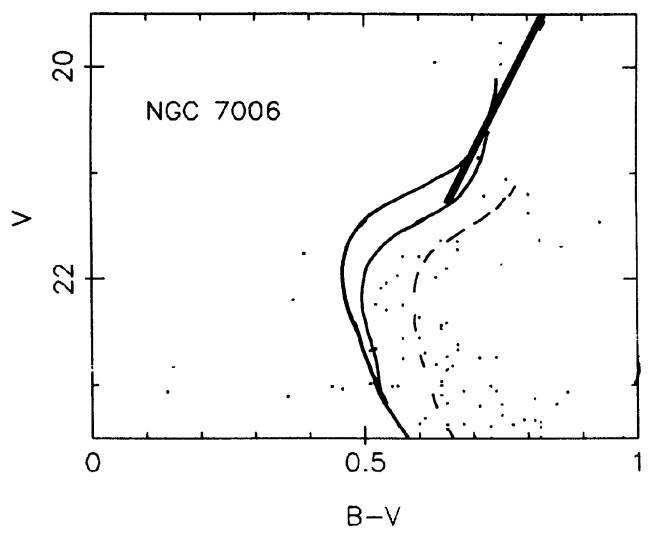

FIG. 11. B,V c-m diagram for NGC 7006. The thick solid line is the mean locus of the bright giants from our frames. Isochrones of age 14 and $18 \times 10^{9} \mathrm{yr}$ with $Z=3 \times 10^{-4}$ and $Y=0.20$ from VandenBerg and Bell (1985) are shown as the thin lines. The dashed line is the $18 \times 10^{9} \mathrm{yr}$ isochrone shifted 0.10 mag redder in $B-V$. measured from low-resolution spectra of individual stars by several authors, including Searle and Zinn (1978) and Cohen and Frogel (1982). A mean value of $[\mathrm{Fe} / \mathrm{H}]=-1.7 \mathrm{dex}$ with respect to the Sun is adopted here. Therefore the $Z=3 \times 10^{-4}$ and $Y=0.20$ isochrones for ages of 14 and 18 billion years from VandenBerg and Bell (1985) are shown as the thin, solid line in the figure. The $18 \times 10^{9} \mathrm{yr}$ isochrone was shifted $0.10 \mathrm{mag}$ to the red in $B-V$ color to yield the dashed line (which is identical to the isochrone fit to NGC 6229) in Fig. 9. This isochrone produces a satisfactory fit for both clusters. The age of NGC 7006 is therefore $17 \pm 2$ billion years. The claim by Carney and Inman (1982) and Carney (1984) of an unusually bright turnoff in NGC 7006, and hence an age significantly below that of most globular clusters, is spurious. (The age uncertainties quoted up to this point reflect only the observational uncertainties in the photometry and the sampling statistics of small numbers of stars, particularly in NGC 7006.)

Globular cluster ages determined by main-sequence fitting are also sensitive to errors in metallicity and helium abundance. Iben (1971) gives approximations for the dependence of the age deduced from the turnoff luminosity on these two parameters. If the uncertainty in metallicity is \pm 0.3 dex and in the helium abundance is \pm 0.05 , then the resulting uncertainties in age are $\pm 7 \%$ and $\pm 10 \%$, respectively, for a cumulative uncertainty (including the observational one) of $\pm 17 \%$ in the cluster age. This does not yet include the errors in the theoretical calculations of the isochrones, which are discussed in many references (see, for example, Flannery and Johnson 1982).

\section{b) Luminosity Functions}

We have seen that isochrone fitting requires a shift in color of about $0.10 \mathrm{mag}$ between the observed and theoretical main sequences. The origin of this problem could be in the data, in the theoretical evolutionary tracks for low-mass metal-poor stars, or in the transformation of $g-r$ to $B-V$ colors. However, the existence of the turnoff itself is obvious in the data, both in color and in relative numbers of stars at various luminosities. One can therefore use the luminosity function as suggested by Paczynski (1984) to determine cluster ages, since presumably the theoretical luminosities are 
more accurate than the colors, and the transformation from calculated to observed parameters is more straightforward. Even if one does not trust the theoretical calculations, relative ages for several clusters of similar metallicity (such as the three studied here) can be obtained since reliable distance moduli are available from RR Lyrae variables in each cluster.

Since our detection criteria, at least within the small outlying fields, are not affected by crowding, and all stars brighter than the limiting magnitude of the tables have been counted, the necessary data for constructing luminosity functions are available (Table VI) . Stars were counted in bins 0.2 mag wide in $g$ in the outlying fields of each cluster. Counts were also done in the more crowded inner fields for NGC 6229 and NGC 7006, where the magnitude limit was set brighter by an amount sufficient to avoid missing any stars, although the magnitudes themselves in these more crowded fields have 0.10 mag uncertainties. In the outlying fields of NGC 7006, an approximate correction for field stars was made only to the faintest four bins by eliminating stars with $B-V \geqslant 0.85$ mag. This is undoubtedly a slight underestimate of the actual field-star contamination; in particular some of the brightest stars in the outlying fields are also probably not members of NGC 7006.

The luminosity functions for NGC 6229 and NGC 7006 were assembled by forcing the bright counts to fit smoothly onto the deeper ones in each cluster. The derived luminosity function for NGC 7006 is shown in Fig. 12, where the luminosity function of the bright stars from the inner fields is indicated by the dashed line. The luminosity function of

TABLE VI. Luminosity functions.

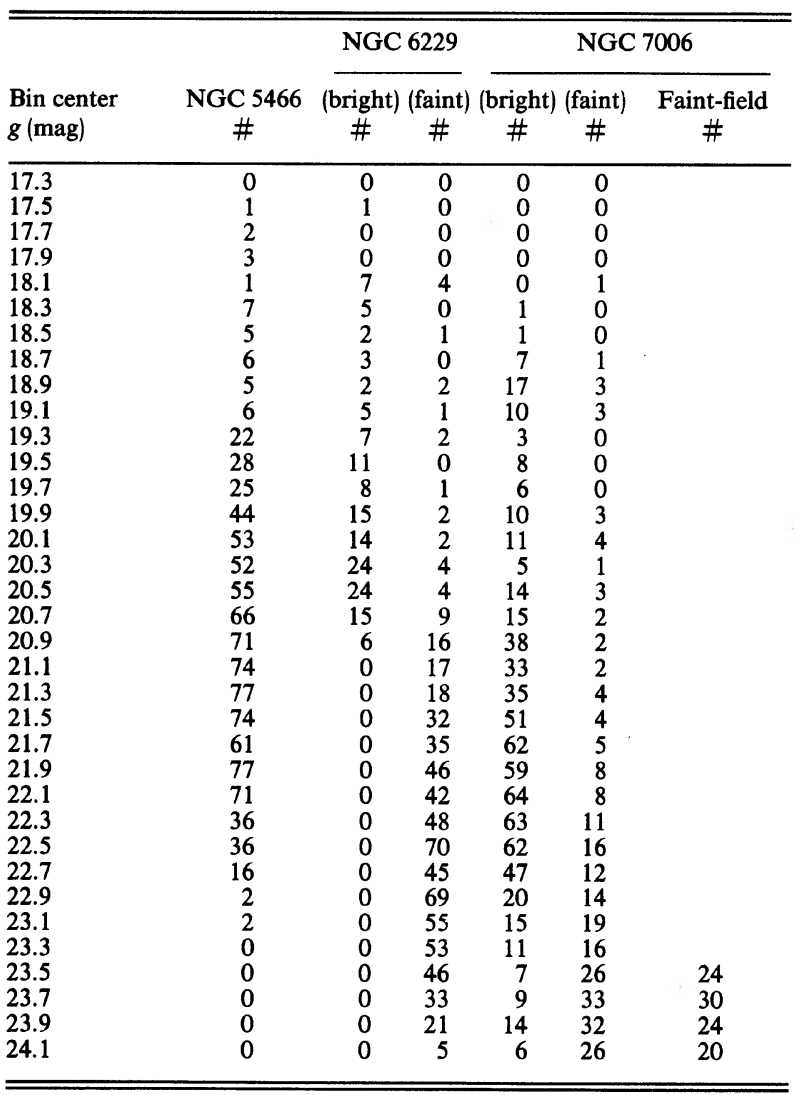

NGC 5466, which is well defined and smooth because of the large number of stars counted, is shifted horizontally by $\delta D M(5466-7006)$, the difference in distance modulus between NGC 5466 and NGC 7006, and vertically by forcing good agreement at intermediate magnitudes $(20 \leqslant g \leqslant 21.5$ mag in NGC 7006) to remove the effect of the difference in the number of stars counted. The scaled luminosity function of NGC 5466 for $\delta D M(5466-7006)$ of $2.6 \mathrm{mag}$ is shown as the solid line, while the short-dashed line (which coincides with the solid line when it is not separately drawn) indicates the luminosity function of NGC 5466 for $\delta D M(5466$ $7006)=1.8 \mathrm{mag}$. The NGC 7006 counts (indicated by points), corrected for field stars (the asterisks), demonstrate that the best fit is $\delta D M(5466-7006)=2.2 \mathrm{mag}$. Although the statistical uncertainties arising from the small number of stars counted in NGC 7006 are apparent in Fig. 11, the cluster's luminosity function supports $\delta D M(5466$ $7006)=2.2 \pm 0.2 \mathrm{mag}$. The true value of $\delta D M(5466-7006)$ as determined from RR Lyrae variables in each cluster is $2.16 \mathrm{mag}$. Unfortunately, the limited number of stars counted and the uncertain field-star corrections do not allow a more stringent limit on age differences to be set than that corresponding to a maximum difference in turnoff luminosity of 0.2 mag between NGC 5466 and NGC 7006, equivalent to a maximum age difference of $+25 \%,-20 \%$.

The luminosity function of NGC 6229 is better determined due to the larger number of stars counted. We find $\delta D M(5466-6229)=1.6 \pm 0.1 \mathrm{mag}$. The true value of the difference in distance moduli is $1.54 \mathrm{mag}$, which should be increased by about 0.1 mag to allow for the slightly higher metallicity of NGC 6229 than NGC 5466. (The correction follows from the approximate parametrizations of the dependence of ages deduced from turnoff luminosities on metallicity by Iben 1971.) Thus the age difference between NGC 6229 and NGC 5466 must be less than $10 \%$ of $18 \times 10^{9}$ yr.

Although no use is made here of Paczynski's (1984) theo-

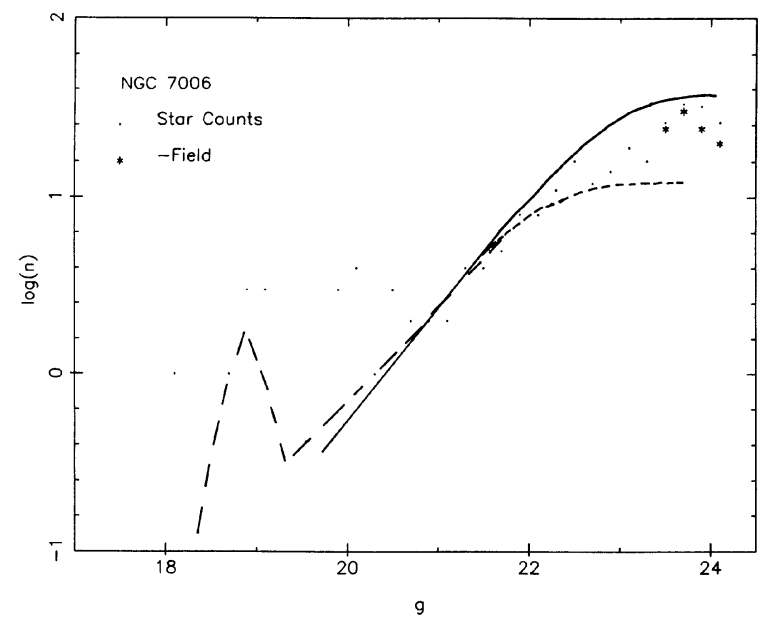

FIG. 12. The logarithm of the number of stars in bins 0.2 mag wide in the fields on the outskirts of NGC 7006 is indicated as a function of $g$ magnitude by dark circles. The asterisks are the star counts corrected for field-star contamination. The dashed line is the luminosity function derived for the brighter stars from fields closer to the center of the cluster. The solid line is the NGC 5466 luminosity function shifted for a difference in distance modulus between that cluster and NGC 7006 of $2.6 \mathrm{mag}$, while the short-dashed line is for a difference of $1.8 \mathrm{mag}$. 
retical predictions for luminosity functions, the shape of the luminosity functions derived from the star counts is similar to that predicted. For nearby clusters where adequate numbers of stars can be obtained without excessive field-star contamination, the technique of age dating via luminosity functions shows great promise.

\section{c) The Horizontal Branch}

The horizontal branch (HB) of NGC 5466 is illustrated in the study of Buonanno et al. (1984). It is quite blue, but without the extended tail of extremely hot, blue stars shown by M92 or M15. The mean color of the HB in NGC 5466 is normal for a cluster of its own metallicity. NGC 7006, on the other hand, is the paradigm of the "second parameter" effect described by Sandage and Wildey (1967), where the HB is unusually red for the metallicity of the cluster. Our c-m diagram for NGC 7006 (Fig. 7) shows an HB identical to that of Sandage and Wildey; there is no evidence for a tail of extremely blue stars, which would have been below the magnitude limit of their data. The morphology of the HB of NGC 6229 is shown for the first time in Fig. 6. It is an unusually red $\mathrm{HB}$ for a relatively metal-poor cluster, with a mean color, including any $\mathrm{RR}$ Lyrae variables, of about $B-V=0.5$ mag. Thus NGC 6229 is an even more extreme example of the second parameter problem than is NGC 7006. Freeman and Norris (1981) point out that the problem of anomalous HB color distribution is not confined exclusively to the classical second-parameter clusters in the outer halo. The distribution of stars in color along the HB is quite different even between well-studied nearby clusters of similar metallicity such as M92 and M15, where the mean color of the HB may be almost identical.

A theoretical review of the factors influencing the color distribution along the HB in globular clusters has been given by Renzini (1977). Age differences of less than 2 billion years in intermediate-metallicity clusters are adequate to produce the range of $\mathrm{HB}$ morphologies observed among such clusters, as could a range in helium of $\Delta Y=0.03$, in $\mathrm{CNO} / \mathrm{Fe}$, or in mean stellar rotation. Peterson (1984) has recently presented observational evidence in support of the hypothesis that rotation is an important factor in HB morphology, while Searle and Zinn (1978), among others, have argued that age differences are the dominant factor.

As we have seen above, such a small range in age is extremely difficult to rule out, given uncertainties in metallicity (and reddening when it is somewhat larger than in the present cases) and photometric uncertainties arising largely from crowding problems.

\section{STAR COUNTS}

Star counts as a function of distance from the cluster center in a globular cluster can be used to derive its tidal radius, which in turn can constrain the potential field in the outer halo of the Galaxy and the cluster's orbit. Peterson and King (1975) discuss the theoretical and observational techniques for determining tidal radii as applied to photographic data.

To facilitate star counts, each frame was taken with the center of the cluster slightly offset from the center of the frame. Thus, although the 4 shooter field is a square whose sides are 8.9 arcmin, star counts could be obtained to radii of almost 7 acmin with two of the chips having good coverage of the central region of the cluster, and the two others covering the periphery of the cluster. Thus, for example, full annular coverage for NGC 6229 is available for $r \leqslant 145 \mathrm{arcsec}$, and coverage over more than $85 \%$ of the full annulus is available for $r \leqslant 240$ arcsec. More than one quarter of the full annulus is present for $r \leqslant 380$ arcsec, which is close to the largest radius for which counts are actually given here. Similar, but not identical, fractions of areal coverage as a function of radius hold for NGC 7006.

The same point-source-detection software described in Sec. II was used on the $600 \mathrm{~s} g$ exposures to generate star counts as a function of radius from the cluster center, but the threshold for detection was set somewhat higher (equivalent to $g \leqslant 23.4 \mathrm{mag}$ ) than that used for the color-magnitude diagrams. The area within 0.5 arcmin of the cluster center was ignored, but otherwise the whole area of each chip was surveyed for point sources. 1931 point sources were detected in NGC 7006 and 2528 in NGC 6229 above the threshold level and below the saturation level of the detector. NGC 5466 is too extended to reach the background field within a single 4Shooter frame, and we do not present any such data for this cluster. The number of detected stars within each chip as a function of radius was corrected for the fraction of the annulus at each radius that was missing from that particular chip to derive the final stellar surface densities. The counts for each chip were kept separately, although each is scaled to full areal coverage. The stellar surface density became approximately constant beyond $r=5.3$ arcmin in both clusters, and the mean value at larger $r$ was taken to be the background value. (This may slightly overestimate the background; ideally it should be determined at even larger radii.) The star counts, corrected for the background, for NGC 6229 and NGC 7006 are presented in Fig. 13, where the stellar surface density $(f)$ is given in units of stars per 100 $\operatorname{arcsec}^{2}$. Each of the four chips is plotted at all radii for which data were available, with a correction factor for missing area of less than 25. There is no separation in Fig. 13 between the individual chips. However, it is apparent from examining the locations of the detected objects superposed on an image of each cluster that the counts in the innermost region are underestimated due to crowding. This effect is also clearly visible in the figure.

King et al. (1968) published visual star counts for these two clusters, which were used by Peterson and King (1975) to derive their tidal radii. The limiting magnitude of the photographic plates used for NGC 6229 was about 21 in $B$ (although one should not imagine good efficiency in visually counting stars down to the plate limit), and a total of 1197 stars were counted, many of which would be too bright to be included in our counts. Less than 650 stars were counted in NGC 7006. Their star counts do not extend out to larger radii from the cluster center than those given here. To directly compare the two data sets, we have scaled King et al.'s star counts to ours by the ratio of the background values adopted by King et al. (1968) to those we choose for each of the clusters. (An error in the scaling, perhaps due to a variation of the luminosity function of the background field with respect to the cluster stars, would uniformly shift the King et al. curve vertically in Fig. 13.) The resulting scaled counts from King et al. (1968) are plotted in Fig. 13 as light circles. The comparison is somewhat sobering, especially when one realizes that our most likely error is an overestimation of the background, which would uniformly lower vertically the light circles representing the King et al. counts in Fig. 8, and would underestimate the stellar density for our counts by the amount the background was underestimated, an effect particularly important in the outermost parts of each cluster.

NGC 6229 is clearly as large as our star counts indicate. 

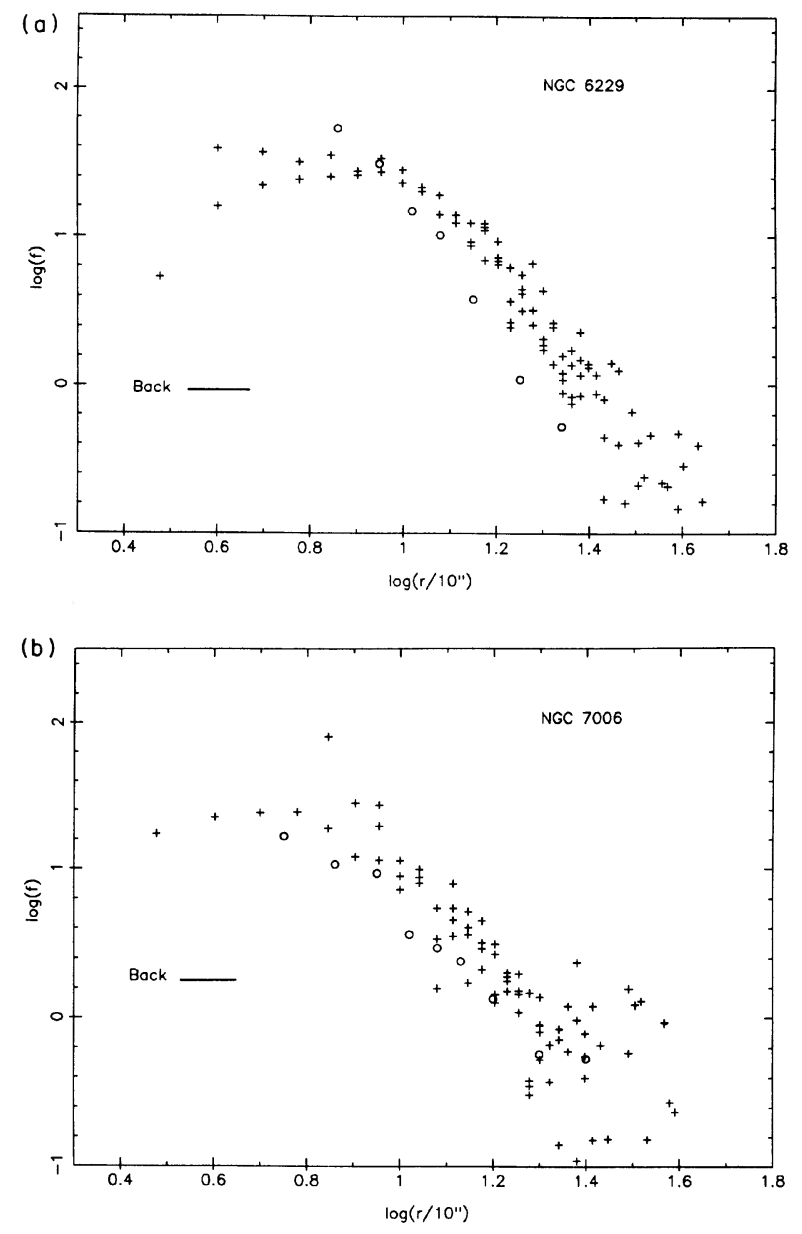

FIG. 13.(a),(b) The surface density of stars in NGC 6229 and NGC 7006 corrected for a background stellar density is shown as a function of distance from the cluster center. The crosses represent the corrected results for each individual 4-Shooter chip. The background subtracted is indicated by the solid horizontal line. The light circles represent the counts of King et al. (1968) scaled to the same background stellar density.

The most distant corner of Field 5 in NGC 6229, shown in Fig. 2, is 275 arcsec from the cluster center. Nearly all of the stars in this field fit onto the cluster c-m diagram and hence must be cluster members. This radius is beyond the outer edge of the outermost annulus of the King et al. counts. In NGC 6229, one sees a clear hint that the manual counts from photographic plates had a systematic dependence of the magnitude range included with radius, so that the spatial extent of the cluster was underestimated. It is of course also possible that the differences in radial distribution are due to mass segregation; the sense of the difference is correct since the King et al. (1968) counts included mostly giants, while ours are dominated by stars of somewhat lower mass. However, a similar analysis of the giants in NGC 6229 from our $136 \mathrm{~s} g$ 4-Shooter frame using a bright magnitude cutoff led to radial distributions for 343 stars. These giants showed the same radial distribution as did the fainter stars, although they did not show the rolloff due to crowding in the central area as severely as did the faint star counts. It is thus likely that the older star-count data are faulty, and that the tidal radius of NGC 6229, in particular, has been underestimated.

\section{SUMMARY}

Color-magnitude diagrams of three distant galactic globular clusters (NGC 5466, 6229, and 7006) have been given in the Thuan-Gunn $g, r$ photometric system, and transformed to the Johnson $B, V$ system. The magnitudes are measured in boxes 1.65 arcsec on a side centered on each point source; offsets determined from well-isolated stars are used to convert the box magnitudes to large-aperture photometry. The photometry reaches below the main-sequence turnoff in all three clusters. Comparison with the isochrones of VandenBerg (1983) and of VandenBerg and Bell (1984) demonstrate that the ages of these three clusters are indistinguishable from those of the better-studied nearby globular clusters; the claims of Carney and Inman (1982) and Carney (1984) of an exceptionally bright turnoff in NGC 7006 are spurious.

Computer-generated star counts given in Sec. III $b$ for the three clusters are used to generate luminosity functions by patching together counts in the crowded inner regions, which contain many bright stars, and in the outlying fields, where the limiting magnitude is deeper. The luminosity functions indicate that the maximum difference in age between NGC 5466 and NGC 6229 is $\pm 10 \%$ of the age, while it is $\pm 25 \%$ for NGC 5466 and NGC 7006 .

The horizontal branch of NGC 6229 is unusually red; it is an even more extreme example of the second parameter problem than NGC 7006. The predicted differences in age between globular clusters that are required to produce the observed variations in horizontal-branch morphology (i.e., the second parameter effect) are too small to be detected in the present data.

NGC 6229 is more extended than was found from King $e t$ al.'s (1968) visual star counts. The tidal radius for NGC 6229 obtained by Peterson and King (1975) from the old starcount data is also probably somewhat underestimated.

This research was supported by NSF Grant No. AST 8212270. I am grateful to Alain Porter for a useful software routine, and especially to Keith Shortridge, who wrote the basic image-processing software package in use at Caltech. To Jim Gunn and the entire crew who built the 4-Shooterbravissimo. I also thank the referee for critical and thoughtful comments on the original version of this manuscript that forced me not to be lazy.

\section{REFERENCES}

Baade, W. (1945). Astrophys. J. 102, 17.

Blanco, V. M., Demers, S., Douglass, G. G., and Fitzgerald, M. P. (1968).

Publ. U. S. Naval Obs. 21, 1968.

Buonanno, R., Buscema, G., Corsi, C. E., Iannicola, G., and Fusi-Pecci, F. (1984). Astron. Astrophys. Suppl. 56, 79.

Burstein, D., and Heiles, C. (1983). Astrophys. J. Suppl. 54, 33.

Carney, B. W. (1984). Publ. Astron. Soc. Pac. 96, 841.
Carney, B. W., and Inman, R. T. (1982). Bull. Am. Astron. Soc. 14, 878. Cohen, J. G., and Frogel, J. A. (1982). Astrophys. J. Lett. 255, L39. Cuffey, J. (1961). Astron. J. 66, 71.

Flannery, B. P., and Johnson, B. C. (1982). Astrophys. J. 263, 166.

Freeman, K. C., and Norris, J. (1981). Annu. Rev. Astron. Astrophys. 19, 319.

Frogel, J. A., Cohen, J. G., and Persson, S. E. (1983). Astrophys. J. 275, 773. 
Gunn, J. E., Carr, M., Chang, J., Danielson, G. E. A., Lorenz, E. O., Lucinio, R., Nenow, V. E., Smith, O. J., Westphal, J. A., and Zimmerman, B. A. (1984). Bull. Am. Astron. Soc. 16, 447.

Harris, W. E., and Racine, R. (1979). Annu. Rev. Astron. Astrophys. 17, 241.

Iben, I., Jr. (1971). Publ. Astron. Soc. Pac. 83, 697.

Kent, S. M. (1985). Publ. Astron. Soc. Pac. 97, 165.

King, I. R., Hedemann, E., Jr., Hodge, S. M., and White, R. E. (1968). Astron. J. 73, 456

Paczynski, B. (1984). Astrophys. J. 284, 670.

Peterson, C. J., and King, I. R. (1975). Astron. J. 80, 427.

Peterson, R. (1984). Astrophys. J. Lett. 294, L35.
Renzini, A. (1977). In Advanced Stages of Stellar Evolution, edited by P. Bouvier and A. Maeder (Geneva Observatory, Geneva), p. 151.

Sandage, A. R. (1970). Astrophys. J. 162, 841.

Sandage, A. R., and Wildey, R. (1967). Astrophys. J. 150, 469.

Schneider, D. P., and Gunn, J. E., and Hoessel, J. G. (1983). Astrophys. J. 264,337.

Searle, L., and Zinn, R. (1978). Astrophys. J. 225, 357.

Thuan, T. X., and Gunn, J. E. (1976). Publ. Astron. Soc. Pac. 88, 543. VandenBerg, D. A. (1983). Astrophys. J. Suppl. 51, 29.

VandenBerg, D. A., and Bell, R. A. (1985). Astrophys. J. Suppl. 58, 561.

Zinn, R. J. (1980). Astrophys. J. Suppl. 42, 19. 